\title{
Calibração: uma abordagem bayesiana
}

\author{
Márcia D’Elia Branco
}

TESE APRESENTADA

$\mathrm{AO}$

INSTTITUTO DE MATEMÁTICA E ESTATÍSTICA

DA

UNIVERSIDADE DE SÃO PAULO

PARA OBTENÇÃO DO GRAU DE DOUTOR

EM ESTATÍSTICA

ORIENTADOR: Profa. Dra. Pilar Loreto Iglesias Zuazola CO-ORIENTADOR: Prof. Dr. Heleno Bolfarine

SÃO PAULO, AGOSTO DE 1997 


\section{Calibração: uma abordagem bayesiana}

Este exemplar corresponde à redação final da tese devidamente corrigida e apresentada por Márcia D’Elia Branco e aprovada pela comissão julgadora.

São Paulo, 20 de agosto de 1997.

\section{Banca Examinadora:}

Profa.Dra. Pilar Loreto Iglesias Zuazola - PUC - Chile

Prof.Dr. Sérgio Wechsler - IME - USP

Prof.Dr. Reinaldo Boris Arellano Valle - PUC - Chile

Prof.Dr. Dani Gamerman - IM - UFRJ

Prof.Dr. Jorge Alberto Achcar - ICMSC - USP 


\section{Resumo}

Neste trabalho apresentamos soluções para o problema de calibração controlada sob a perspectiva bayesiana da inferência estatística. Em primeiro lugar, tratamos do problema sob a suposição de linearidade e de que os erros são distribuídos de acordo com uma distribuição elíptica. Para o modelo elíptico dependente mostramos que a distribuição a posteriori de interesse coincide com a distribuição a posteriori obtida sob a suposição de normalidade, quando considerada uma distribuição a priori imprópria para o parâmetro de dispersão. Uma análise conjugada também é apresentada. Entretanto, não obtemos essa coincidência de resultados para o modelo independente. Neste caso, a distribuição a posteriori deverá depender do particular modelo elíptico especificado. Considerando algumas especificações a priori e a representabilidade do modelo elíptico. obtivemos formas gerais para estas distribuições a posteriori, caracterizando-as como mistura de distribuições conhecidas. Além disso, foram obtidas formas conhecidas para todas as distribuições a posteriori de um parâmetro, condicionais aos demais, possibilitando a implementação do amostrador de Gibbs. Posteriormente, tratamos do problema de calibração sem a suposição de linearidade e considerando que a variável resposta é categorizada. Apresentamos uma generalização do conhecido modelo probit, onde a função de ligação é uma distribuição elíptica. Nesse caso, obtivemos uma aproximação assintótica para a distribuição a posteriori, bem como uma solução via método MCCM (Monte Carlo baseado em Cadeias de Markov), para o modelo binomial. Para o modelo multinomial, propomos a solução via MCCM e apresentamos formas conhecidas para todas as distribuições condicionais. 


\section{Abstract}

In this thesis, we present solutions for the controled calibration problem under the Bayesian perspective to statistical inference. The first problem considered is related to the linear case with elliptically distributed errors. For the elliptical dependent model it is shown that the posterior distribution for the quantity of interest coincides with the posterior distribution obtained under the normality assumption with an improper prior distribution. A conjugate prior analysis is also considered. The coincidence of the inference is not noted for the independent elliptical model. As consequences of some prior specifications and representability of the elliptical model, general expressions are obtained for the posterior distributions, which are characterized as mixtures of known distributions. Moreover, simple conditional posterior distributions of one parameter given the others were obtained which allowed the use of the Gibbs sampler to obtain approximations to the posterior distributions of the quantities of interest. Subsequently, the calibration problem for nonlinear situations was considered under the assumption that the response variable is categorized. A generalization of the well know probit model was considered, by taking an elliptical distribution function as the link function. For this case, an asymptotic approximation was obtained for the posterior distribution. A MCMC (Monte Carlo Markov Chain) solution is considered for the binomial model. For the multinomial model, a MCMC solution is present and the conditional distributions required for implementing the approach are obtained. 


\section{AGRADECIMENTOS}

Agradeço a minha orientadora, Profa.Dra. Pilar Iglesias, que mesmo tendo assumido a tarefa de orientação a apenas alguns meses e. de vivermos em países diferentes, teve um papel fundamental no desenvolvimento deste trabalho. Suas sugestões e seu interesse foram essenciais para a conclusão desta tese. Admiro sua conduta profissional e a paixão que demonstra pela pesquisa. Além do prazer do trabalho conjunto com a Pilar, tive a felicidade de conquistá-la como amiga. - Obrigada Pilar!

Agradeço, também, a contribuição do Prof.Dr. Heleno Bolfarine, meu co-orientador. Conheço o Prof. Heleno há muitos anos, mas somente nestes últimos dois anos tive a oportunidade de aprender um pouco mais sobre sua conduta como pesquisador. Admiro sua capacidade de pesquisa e sua presteza na colaboração com os outros, particularmente para com os alunos de pós-graduação deste Instituto.

Agradeço ao Prof.Dr. Reinaldo Arellano-Valle, pela gentileza de ler meu trabalho e pelas diversas sugestões. Sua colaboração na obtenção dos resultados do capítulo 3 foram fundamentais. Mais uma vez, no decorrer deste doutorado, tive a felicidade de encontrar um ser humano excepcional e conquistar um amigo.

Agradeço, também, o Prof. Dr. Carlos Alberto de Bragança Pereira, pela sugestão do tema de tese e pela contribuição na primeira fase deste trabalho, bem como, na minha formação bayesiana. Admiro no Carlinhos seu otimismo e agradeço a confiança depositada na minha pessoa.

De um modo geral, gostaria de agradecer ao Departamento de Estatística da USP pela formação geral que me proporcionou. Penso que um processo de doutoramento não restringe-se a apresentação de uma tese, mas visa uma formação mais ampla do indivíduo. Os cursos e principalmente os seminários, em especial, os de Fundamentos de Inferência Estatística coordenados pelo Prof.Dr. Sérgio Weschler, contribuiram fortemente nesta formação.

Mais uma vez devo agradecer ao Departamento de Estatística, agora na qualidade de docente. Em especial, quero agradecer aos colegas Elisabeti Kira, Rinaldo Artes e Antonio Carlos P. Lima, por diversas vezes terem sanado minhas dúvidas de $\mathrm{ATT}_{\mathrm{E} X}$, S-Plus e outros probleminhas com a máquina. A amizade de vocês ajudou a tornar mais suportável certos 
momentos de crise. Em especial, agradeço a Beti, minha colega de sala, e ao grupo de Sistemas de Partículas por terem cedido um PC pra trabalho em minha residência e também permitido o uso, e abuso, do X-terminal em nossa sala.

Agradeço ao Projeto Andes C-12777/9 de colaboração academica-cientifica entre Brasil e Chile e ao Projeto Fondecyt 1971128, pelo apoio financeiro.

Finalmente, devo agradecer a minha família. Aos meus pais, cujo tempo e distância só fizeram com que o afeto e admiração que sinto pelos "véios", Alcides e Carlota, cresça ainda mais. Ao Chico e a Iara, que mais uma vez me acolheram em sua casa num momento de necessidade, e pelos inúmeros almoços de domingo. Tenho que agradecer a todos que dividiram comigo a tarefa de mãe, em particular, a Creche da USP e a Iracema. Em especial, agradeço ao pai do meu filho, Társis, pelo incentivo e carinho durante os anos que estivemos juntos; e por ter assumido, nestes últimos meses, a dupla tarefa de pai e mãe.

Ao meu filho Gustavo não devo somente agradecer, por tudo de bom que ele representa pra mim, mas devo pedir desculpas pelo tempo que não pudemos estar juntos. Em minha defesa envoco o amor que sinto pelo meu trabalho, só não sendo maior do que aquele que sinto por ele.

Márcia

São Paulo, junho/97 


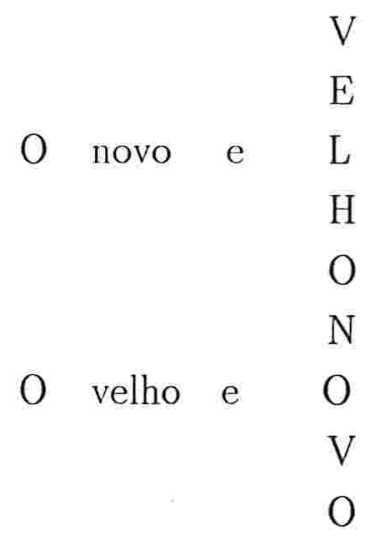

Ao Gustavo $\mathrm{e}$

Alcides

O homem velho é o rei dos animais 


\section{Sumário}

1 Introdução $\quad 3$

2 Calibração linear no modelo normal $\quad 7$

2.1 Soluções clássicas . . . . . . . . . . . . . . . . . . 7

2.1.1 Estimador clássico vs estimador inverso . . . . . . . . . . . 7

2.1 .2 Intervalo de confiança . . . . . . . . . . . . . . . . 10

2.2 Soluções bayesianas . . . . . . . . . . . . . . . . . . . . . . . 13

3 Calibração linear no modelo elíptico $\quad 23$

3.1 Introdução . . . . . . . . . . . . . . . . . . . . . . . . . . 23

3.2 Distribuições elípticas . . . . . . . . . . . . . . . . . . . 24

3.3 O modelo de regressão linear elíptico . . . . . . . . . . . . . . . . . . . 29

3.4 Calibração no modelo elíptico dependente . . . . . . . . . . . . . . . 35

3.5 Calibração no modelo elíptico independente . . . . . . . . . . . . . . . . . 39

3.6 Medindo o comprimento de uma maratona . . . . . . . . . . . . . . 50

4 Extensões multivariadas para o modelo de calibração elíptico $\quad \mathbf{5 4}$

4.1 Introdução . . . . . . . . . . . . . . . . . . . . . . 54

4.2 As matrizes elípticas . . . . . . . . . . . . . . . . . . . . . 55

4.3 O modelo elíptico dependente multivariado . . . . . . . . . . . . . . . . . 59

$4.4 \mathrm{O}$ modelo elíptico independente multivariado . . . . . . . . . . . . . . 61

5 Calibração em dados categorizados $\quad 67$

5.1 Introdução . . . . . . . . . . . . . . . . . . . . . . 67

5.2 O modelo binomial . . . . . . . . . . . . . . . . . . 69

5.2.1 Estimação da distribuição a posteriori via algoritmo estocástico $\quad$. 70

5.2.2 Aproximação assintótica para a distribuição a posteriori . . . . . . 78 
5.3 O modelo multinomial . . . . . . . . . . . . . . . . . 81

5.3 .1 Categorias ordenadas . . . . . . . . . . . . . . 82

5.3 .2 Categorias não ordenadas . . . . . . . . . . . . . . 84

5.4 Análise de dados citogenéticos . . . . . . . . . . . . . . . . . 85

6 Considerações finais $\quad 92$

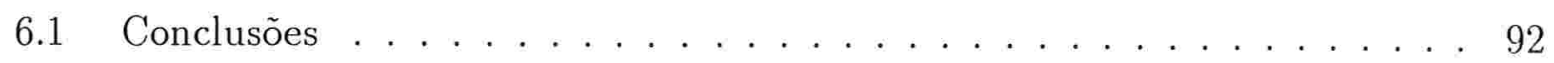

6.2 Perspectivas futuras . . . . . . . . . . . . . . . . . . 94

A O Amostrador de Gibbs $\quad 96$

A.1 Introdução . . . . . . . . . . . . . . . . . . . . . . . 96

A.2 Critérios de convergência . . . . . . . . . . . . . . . . . . . . 98

A.3 Comparação com a algoritmo DA . . . . . . . . . . . . . . . 100

B Gráficos $\quad 102$ 


\section{Capítulo 1}

\section{Introdução}

O problema estatístico de calibração é motivado pelo problema de calibração em um laboratório científico. Suponha que existam neste laboratório dois instrumentos de medição, A e B. De modo que, o instrumento A seja extremamente preciso, mas de alto custo e o instrumento $\mathrm{B}$, ao contrário, seja menos preciso e de baixo custo ( este custo não é necessariamente monetário). O problema é recuperar as medidas "verdadeiras" (instrumento A) a partir da observação das medidas do instrumento B. Para tal, é construída o que costuma-se denominar curva de calibração, que é uma função que relaciona as medidas dos diferentes instrumentos. Para construção da curva de calibração são fixadas $n$ unidades cujas medidas feitas pelo instrumento $\mathrm{A}$ são conhecidas, e repete-se a medição destas unidades utilizando-se o instrumento B. Critérios estatísticos devem ser utilizados para a construção dessa curva e para a predição das medidas desconhecidas. A modelagem estatística deste problema ficou conhecida como um problema de calibração, também denominado por alguns autores como um problema de regressão inversa. No entanto, o problema estatístico de calibração não fica restrito ao problema de calibração de instrumentos, como descrito acima.

Uma outra ilustração do problema de calibração é derivada da citogenética. Considere um acidente nuclear fictício onde um trabalhador sofre uma exposição a uma dose de radiação desconhecida, $D_{0}$. O efeito citogenético, $y_{0}$, do acidente é medido no sangue do paciente. Para "estimar-se" o valor de $D_{0}$, um experimento de calibração é planejado. Sangue de outros trabalhadores (colegas que trabalham no mesmo ambiente daquele que sofreu a exposição), que não sofreram o mesmo tipo de acidente, é coletado. Após o preparo de n lâminas com este sangue, as mesmas são expostas a diferentes níveis conhecidos de 
radiação, digamos $d_{1}, d_{2}, \ldots, d_{n}$. Os efeitos citogenéticos dessas exposições são observados e obtem-se os valores $y_{1}, y_{2}, \ldots, y_{n}$. Note que $y_{0}$ é uma medida in vivo enquanto os $y_{i}, 1 \leq$ $i \leq n$, são medidas in vitro .

A situação descrita acima é denominada na literatura como um problema de calibração controlada, pois no experimento in vitro os valores das doses são previamente fixados. Entretanto, se os valores $d_{i}^{\prime} s$ forem vistos como realizações de uma quantidade aleatória, o problema é conhecido como calibração natural. O problema de calibração natural pode ser modelado pensando-se na distribuição conjunta para o par $(D, Y)$ e neste caso não há diferença em falar da predição de $Y$ ou de $D$, basta obter o condicionamento adequado. Entretanto, num problema de calibração controlada o modelo é sempre condicional de $Y$ dado um valor $d$ (fixado), e a inversão deste condicionamento só terá sentido na medida in vivo, quando buscamos o valor desconhecido $d_{0}$, após a observação de um valor $y_{0}$. Assim, em calibração controlada $Y=f(d)+e$, onde $e$ é um erro aleatório associado a quantidade $Y$, e para as quantidades fixadas $d_{1}, d_{2}, \ldots, d_{n}$ não temos nenhum erro asssociado. É sob estas condições que iremos desenvolver nosso estudo.

No capítulo 2 apresentamos uma breve revisão da literatura em calibração controla$\mathrm{da}$, a qual tem apresentado resultados essencialmente sob as hipóteses de linearidade, normalidade e homocedasticidade dos erros. A abordagem clássica para o problema será discutida brevemente e somente neste capítulo; para uma revisão mais completa ver Osborne (1991) e Brown (1993). A discusão clássica do problema gira em torno da comparação de dois estimadores; o clássico e o inverso. Essa discussão é gerada pelo fato do estimador clássico, que é de máxima verossimilhança sob a hipótese de normalidade, ter esperança e variância infinitas. Além disso, há problemas com o intervalo de confiança clássico, quando o parâmetro de regressão $\beta$ está próximo de zero. Em seguida, apresentamos os resultados bayesianos obtidos por Hoadley (1970) e Hunter and Lamboy (1981) no campo univariado e por Brown (1982) no campo multivariado. Hoadley (1970) provou que o estimador inverso é uma estimador de Bayes, para uma específica distribuição a priori, e apresentou uma forma geral para a distribuição a poteriori de $x_{0}$. Brown (1982) estendeu os resultados de Hoadley (1970) para o campo multivariado.

No capítulo 3 mantivemos a hipótese de linearidade para $f$, mas consideramos os erros numa classe mais ampla de distribuições, a classe das elípticas. Esta classe contêm a distribuição normal e é formada por distribuições que são invariantes por transformações ortogonais. Em relação à hipótese sobre os erros, duas situações são consideradas: o modelo elíptico dependente, onde os erros são conjuntamente elípticos e não correlacionados; 
e o modelo elíptico independente, onde os erros são independentes e identicamente distribuidos com distribuição elíptica. No primeiro caso mostramos que a classe conjugada. associada ao parâmetro de regressão $\beta$, é a classe das elípticas. Além disso, o estimador de Bayes, sob perda quadrática, não depende da especificação do modelo e é uma combinação linear convexa das observações e da média a priori. No problema de calibração verificamos que, sob uma distribuição a priori imprópria para a variância dos erros, as inferências obtidas sob normalidade são mantidas no modelo elíptico dependente. Obtemos assim um resultado robusto no sentido distribucional. No entanto, o mesmo não ocorre quando estamos sob o modelo elíptico independente. Neste caso, obtivemos formas gerais para as distribuições a posteriori de $x_{0}$, considerando-se duas especificações a priori; uma com prioris impróprias e outra na classe conjugada do parâmetro de regressão. Para implementação deste resultado, já que as formas analíticas são de difícil tratamento, recorremos aos métodos MCCM. Utilizamos o amostrador de Gibbs, pois obtivemos formas analiticas fechadas e conhecidas para todas as distribuições condicionais a posteriori. Finalizamos este capítulo com uma aplicação, sob a hipótese de erros t-Student independentes.

Considerando que tanto a quantidade controlada $X$, quanto a resposta $Y$, podem ser vetores, apresentamos, no capítulo 4, extensões multivariadas de alguns resultados do capítulo 3. O desenvolvimento deste capítulo requer a utilização de matrizes aleatórias, em particular, a extensão da definição de elipticidade de um vetor para uma matriz. Utilizamos o conceito de elipticidade a esquerda, dado por Dawid (1977), para definir uma matriz elíptica. No modelo dependente obtemos novamente a robustez, em termos de distribuição, dos resultados obtidos por Brown (1982) sob normalidade. No modelo independente trabalhamos com a classe das distribuições normal Wishart-invertida como priori para os parâmetros de regressão e uma normal multivariada para a quantidade de interesse $x_{0}$, o que nos permite obter formas conhecidas para todas as distribuições condicionais a posteriori.

Finalmente, no capítulo 5 consideramos o caso onde a variável resposta é discreta. Em particular, dois modelos foram considerados: o modelo binomial e sua extensão multivariada, o modelo multinomial. Deste modo, podemos utilizar a denominação de dados categorizados, pois a resposta apenas irá indicar a categoria a que o indivíduo pertence. Para este tipo de modelo, em geral, a suposição de linearidade não é adequada e recorremos a uma regressão logística ou probit. Estes dois modelos de regressão pertencem a uma classe mais geral de modelos, onde a função de distribuição que relaciona as quantidades $Y$ e $X$ é uma distribuição elíptica; pois, tanto a distribuição normal, que caracteriza o 
modelo probit, como a distribuição logística estão na classe das elípticas. Sob este modelo, com função de ligação elíptica, desenvolvemos nosso estudo do problema de calibração. Na seção 5.2.1, sugerimos uma solução numérica para o problema baseada no algoritmo de Tanner and Wong (1987). A idéia é introduzir no modelo variáveis latentes que permitam a obtenção de distribuições condicionais a posteriori conhecidas, para implementação do amostrador de Gibbs. Na seção 5.2.2, obtemos a matriz de informação de Fisher para o problema, e a especificação de uma possível aproximação normal para a distribuição a posteriori de $x_{0}$. Nas seção 5.3 , propomos uma extensão da solução numérica da seção 5.2 , para o modelo multinomial. Finalmente, na seção 5.4 apresentamos uma aplicação na área de citogenética, para o modelo binomial. 


\section{Capítulo 2}

\section{Calibração linear no modelo normal}

\subsection{Soluções clássicas}

\subsubsection{Estimador clássico vs estimador inverso}

O problema de calibração é uma questão bastante antiga em estatística. Por exemplo, Eisenhart (1939) já colocava esta questão, salientando que o objetivo ao estabelecer-se uma relação entre duas variáveis em estudo, $X$ e $Y$, é o de fazer predição. Desse modo, para que a observação de uma das variáveis permita determinar indiretamente o valor da outra, é estabelecida uma relação funcional entre elas, denominada curva de ajuste. Esta baseia-se em um conjunto de observações de $X$ e $Y$, genericamente denotado por $\left(x_{1}, y_{1}\right) ; \ldots ;\left(x_{n}, y_{n}\right)$, onde $n$ é o tamanho da amostra. A obtenção da curva de ajuste depende de suposições a respeito de sua forma funcional e também da aleatoriedade das variáveis em estudo. [Por exemplo, tipicamente é assumido que $X$ é uma variável determinística, isto é, seus valores são previamente fixados pelo pesquisador, e que $Y$ é uma variável aleatória (v.a.), tal que $E[Y \mid X=x]=\alpha+\beta x$. Neste caso, a curva de ajuste será linear do tipo $a+b x$ onde $a$ e $b$ são as usuais estimativas de mínimos quadrados de $\alpha$ e $\beta$, respectivamente, isto é,

$$
a=\bar{y}-b \bar{x} \quad \text { e } \quad b=\sum_{i=1}^{n}\left(x_{i}-\bar{x}\right)\left(y_{i}-\bar{y}\right) / \sum_{i=1}^{n}\left(x_{i}-\bar{x}\right)^{2} .
$$

A predição pode ser feita de duas maneiras:

(i) direta: no sentido direto da regressão, com a predição, de um novo valor para $Y$;

(ii) inversa: no sentido inverso, com a predição de um novo valor para $X$. 
Esta última é denominada regressão inversa ou calibração.

Mantendo-se a suposição de linearidade, $E\left[Y \mid X=x_{0}\right]=\alpha+\beta x_{0}$, se um novo valor $x_{0}$ para $X$ é observado, uma estimativa natural do valor esperado de $Y$ associado com $x_{0}$ é $a+b x_{0}$. No entanto, se o interesse for inverso, isto é, dada uma observação $y_{0}$ de $Y$ e deseja-se predizer o valor de $X$ que produziu $y_{0}$, obtem-se a estimativa clássica, $\hat{x}_{C}$, utilizando-se a mesma reta de ajuste, isto é,

$$
\hat{x}_{C}=\frac{y_{0}-a}{b}=\frac{\left(y_{0}-\bar{y}\right)}{b}+\bar{x},
$$

onde $\bar{y}$ e $\bar{x}$ são as médias amostrais de $y_{1}, . ., y_{n}$ e $x_{1}, \ldots, x_{n}$, respectivamente. Interpretando $a$ e $b$ como resultados de duas variáveis aleatórias (função dos Y's), que denotaremos por $\hat{\alpha}$ e $\hat{\beta}$, respectivamente, temos o estimador

$$
\hat{X}_{C}=\frac{Y_{0}-\hat{\alpha}}{\hat{\beta}}
$$

o qual é denominado estimador clássico.

Usualmente, além da hipótese de linearidade, acrescentam-se as hipóteses de normalidade, homocedasticidade (variâncias iguais) e independência, isto é,

$$
Y_{i} \mid X=x_{i} \stackrel{i n d}{\sim} N\left(\alpha+\beta x_{i} ; \sigma^{2}\right) \quad i=0,1,2 \ldots, n
$$

Nesse caso, utilizando a propriedade de invariância do estimador de máxima verossimilhança(e.m.v.) (Mood, Graybill and Boes 1974) e o fato de que $\hat{\alpha}, \hat{\beta}$ e $Y_{0}$ são os e.m.v. para $\alpha, \beta$ e $E\left[Y_{0}\right]$, respectivamente, é fácil ver que $\hat{X}_{C}$ é o e.m.v para $x_{0}$. Outro modo de escrever este estimador é

$$
\hat{X}_{C}=\bar{x}+\frac{\left(Y_{0}-\bar{Y}\right)}{\hat{\beta}} .
$$

Sob o modelo 2.1, $\hat{\beta}$ tem distribuição normal e é independente de $Y_{0}-\bar{Y}$. Portanto,

$$
E\left[\hat{X}_{C}\right]=\bar{x}+E\left[Y_{0}-\bar{Y}\right] E\left[\frac{1}{\hat{\beta}}\right] .
$$

Mas, $E\left[\frac{1}{\hat{\beta}}\right]$ é infinito, pelo fato de ser o valor esperado da inversa de uma variável normal, ver Piegorsch and Casella (1985). Logo, o valor esperado do estimador clássico é infinito. Além disso, sua variância é infinita. Consequentemente, esse estimador tem erro quadrático médio infinito, isto é, 


$$
E Q M\left(\hat{X}_{C}\right)=E\left[\left(\hat{X}_{C}-x_{0}\right)^{2} \mid \alpha, \beta, \sigma^{2}, x_{0}\right]=\infty .
$$

Este fato fez com que se buscassem estimadores alternativos ao estimador clássico para o problema de calibração,

$>$ Utilizando-se o artifício da regressão de $X$ em $Y$ podemos determinar a reta de mínimos quadrados, $c+d y$, onde

$$
c=\bar{x}-d \bar{y} \quad \text { e } \quad d=\sum_{i=1}^{n}\left(x_{i}-\bar{x}\right)\left(y_{i}-\bar{y}\right) / \sum_{i=1}^{n}\left(y_{i}-\bar{y}\right)^{2},
$$

são as estimativas de mínimos quadrados, isto é, $c$ e $d$ são valores de $\phi$ e $\delta$, respectivamente, que minimizam a expressão

$$
\sum_{i=1}^{n}\left(x_{i}-\phi-\delta y_{i}\right)^{2}
$$

Portanto, a estimativa natural para $x_{0}$ é

$$
\hat{x}_{I}=c+d y_{0} .
$$

Novamente, entendendo $c, d$ e $y_{0}$ como realizações de variáveis aleatórias denotadas por $\hat{\phi}, \hat{\delta}$ e $Y_{0}$, respectivamente, temos o estimador

$$
\hat{X}_{I}=\bar{x}+\left(Y_{0}-\bar{Y}\right) \hat{\delta}
$$

o qual é denominado estimador inverso.

Considerando que as retas de ajuste não são coincidentes e portanto obtem-se estimativas diferentes para $x_{0}$, dependendo da reta considerada, uma questão que surge naturalmente é qual dos dois estimadores deve ser utilizado.

A utilização do estimador inverso foi desconsiderada por um longo período baseando-se nos argumentos de Eisenhart (1939) que, entre outras coisas, observa que no problema de calibração controlada $X$ não é aleatório e portanto, não tem sentido pensar na regressão de $X$ em $Y$. Mas, o polêmico trabalho de Krutchkoff (1967) buscou argumentos para justificar o uso do estimador inverso. Neste trabalho Krutchkoff tenta mostrar que o fato do estimador clássico ter erro quadrático médio infinito tem consequências práticas importantes. Isto é feito através de um estudo comparativo dos erros quadráticos médios empíricos dos estimadores clássico e inverso, utilizando-se recursos de simulação, concluindo-se pela melhor eficiência do estimador inverso. A partir de então alguns autores tem se preucupado em comparar os dois estimadores, Williams (1969), Krutchkoff (1969), Berkson (1969), 
Hoadley (1970), Chow and Shao (1990), Osborne (1991), Kubokawa and Robert (1994), entre outros.

Mas, ainda que o trabalho de Krutchkoff (1967) tenha revivido a possibilidade da utilização do estimador inverso, ele foi alvo de muitas criticas. Williams (1969) admite e verifica que o estimador inverso tem erro quadrático médio finito, sem argumentos de simulação. Segundo ele, isto não é justificativa suficiente para considerá-lo melhor que o estimador clássico, pois é uma propriedade que qualquer constante possui. A questão é que não há argumentos teóricos, segundo Williams, que justifiquem o estimador inverso. Além disso, ele mostra que nenhum estimador não viesado tem variância finita e portanto o fato do erro quadrático médio do estimador clássico ser infinito não é tão grave. Berkson (1969) chama atenção para a inconsistência do estimador inverso, isto é, ele não converge para o verdadeiro valor de $x_{0}$ quando $n \rightarrow \infty$, a não ser no caso particular de $x_{0}=\bar{x}$. A importância prática desta inconsistência está no fato de que para se construir uma curva de calibração eficiente deve-se contar com muitas observações e neste caso o estimador inverso se mostra menos adequado do que o clássico, pois este último é consistente. Mais tarde o próprio Krutchkoff (1969) admite a superioridade, em relação a ter menor erro quadrático médio, do estimador clássico em relação ao inverso para os casos onde $x_{0}$ esta fora do dominio dos valores $x_{i}^{\prime} s$ da calibração. No entanto, os resultados obtidos anteriormente continuam válidos para $x_{0}$ dentro do dominio dos $x_{i}^{\prime} s$, especialmente quando $x_{0}$ esta próximo de $\bar{x}=\frac{1}{n} \sum_{i=1}^{n} x_{i}$. O primeiro autor a obter uma justificativa teorica para o estimador inverso é Hoadley (1970). Ele considera uma abordagem bayesiana para mostrar que o estimador inverso é um estimador de Bayes. Veremos com detalhes esta abordagem na seção 2.2 .

A discussão a respeito do erro quadrático médio não ser finito não é a unica controvérsia a respeito de $\hat{X}_{C}$. Novos problemas surgem com os intervalo de confiança, ponto que será tratado a seguir.

\subsubsection{Intervalo de confiança}

O intervalo de confiança clássico para o problema de calibração proposto por Eisenhart (1939), foi também derivado por Fieller (1954) através de argumentos fiduciais. A construção deste intervalo baseia-se na distribuição de probabilidades conjunta de $\left(\hat{\alpha}, \hat{\beta}, S^{2}, Y_{0}\right)$, onde 


$$
S^{2}=\sum_{j=1}^{n} \frac{\left(Y_{j}-\hat{\alpha}-\hat{\beta} x_{j}\right)^{2}}{n-2} .
$$

Utilizando-se dos fatos: $\hat{\alpha}, \hat{\beta}$ e $Y_{0}$ tem distribuição normal ; $S^{2}$ tem distribuição quiquadrado com $(n-2)$ graus de liberdades e é independente das demais variáveis. Sabemos que a razão de uma v.a. normal pela raiz de uma v.a. qui-quadrado é uma v.a. com distribuição t-Student com $(n-2)$ graus de liberdades. Então,

$$
T=\frac{\left(Y_{0}-\hat{\alpha}-\hat{\beta} x_{0}\right)}{\left[\frac{S^{2}}{s_{x}^{2}}\left(1+\frac{1}{n}+x_{0}^{2}\right)\right]^{\frac{1}{2}}}
$$

tem distribuição t-Student com (n-2) graus de liberdades, onde $s_{x}^{2}=\sum_{j=1}^{n} \frac{\left(x_{j}-\bar{x}\right)^{2}}{n}$. Portanto,

$$
T^{2}=\frac{s_{x}^{2} \hat{\beta}^{2}\left(\hat{X}_{C}-x_{0}\right)^{2}}{S^{2}\left(1+\frac{1}{n}+x_{0}^{2}\right)}
$$

tem distribuição F-snedecor com 1 e $(n-2)$ graus de liberdades, $F(1, n-2)$ (Mood et al. 1974) .

Denotando por $\gamma$ o coeficiente de confiança, o intervalo de confiança de $100 \gamma \%$ para $x_{0}$ é obtido utilizando-se 2.2 e fazendo $T^{2} \leq f_{1, n-2}(\gamma)$, onde $f_{1, n-2}(\gamma)$ denota o $\gamma$-ésimo percentil da distribuição $F(1, n-2)$. Assim, após algumas simplificações obtemos a forma quadrática :

$$
A x_{0}^{2}-B x_{0}+C \leq 0
$$

onde

$$
A=\hat{\beta}^{2}-\frac{f_{1, n-2}(\gamma) S^{2}}{s_{x}^{2}}, B=2 \hat{\beta}\left(y_{N}-\hat{\alpha}\right) e C=\left(y_{N}-\hat{\alpha}\right)^{2}-\left[\frac{f_{1, n-2}(\gamma) S^{2}}{s_{x}^{2}}\left(1+\frac{1}{n}\right)\right] .
$$

A solução da equação 2.3 pode ser separada em quatro casos distintos:

(i) para $A>0$ e $\left(B^{2}-4 A C\right)>0$, obtem-se duas raízes reais $r_{1}, r_{2}$, e a região de confiança é dada pelo intervalo $\left(r_{1}, r_{2}\right)$;

(ii) para $A>0$ e $\left(B^{2}-4 A C\right)<0$ não existe raiz real e o intervalo de confiança é vazio;

(iii) para $A<0$ e existem duas raízes reais $r_{1}, r_{2}$, o intervalo de confiança é a união de dois intervalos $\left(-\infty, r_{1}\right) \cup\left(r_{2}, \infty\right)$;

(iv) para $A<0$ e não existe raiz real, a região de confiança é a reta toda $\mathbb{R}$. Consequentemente, apenas o caso (i) é razoável como intervalo de confiança. 
Se definirmos $F=s_{x}^{2} \hat{\beta}^{2} / S^{2}$, que é a estatística de teste para hipótese $H_{0}: \beta=0$, a região de confiança para $x_{0}$ é dada pela seguinte região

$$
\begin{cases}\left\{x: r_{1}<x<r_{2}\right\}, & \text { se } F>f_{1, n-2}(\gamma) \\ \left\{x: x \leq r_{1}\right\} \cup\left\{x: x \geq r_{2}\right\}, & \text { se }\left\{\frac{n+1}{n+1+x_{C}^{2}}\right\} f_{1, n-2}(\gamma)<F<f_{1, n-2}(\gamma) \\ \mathbb{R}, & \text { se } F<\left\{\frac{n+1}{n+1+x_{C}^{2}}\right\} f_{1, n-2}(\gamma)\end{cases}
$$

onde $r_{1}$ e $r_{2}$ são iguais a

$$
\frac{F \hat{x}_{C}}{F f_{1, n-2}(\gamma)} \mp \frac{\left\{f_{1, n-2}(\gamma)\left[(n+1)\left(F-f_{1, n-2}(\gamma)\right)+F \hat{x}_{C}^{2}\right]\right\}^{\frac{1}{2}}}{F-f_{1, n-2}(\gamma)}, \quad \text { com } r_{1} \leq r_{2} \text {. }
$$

Portanto a não obtenção de um intervalo satisfatório para $x_{0}$, coincide com a não rejeição da hipótese $H_{0}: \beta=0$. Este fato serve de argumento para alguns autores como, por exemplo, Williams (1969) defender esta região de confiança, já que $\beta=0$ significaria a não existência de uma relação linear entre $X$ e $Y$ e não teria sentido utilizar o estimador $\hat{X}_{C}$, pois os dados não trariam informação pertinente para a estimativa de $x_{0}$. Tal argumento, é criticado por Hoadley (1970) com a justificativa de que em termos bayesianos os dados são não informativos se a sua opinião a priori não se altera diante das observações, isto é, a priori = a posteriori. Além disso, qual o significado do coeficiente de confiança $\gamma$ ? Quando adotamos $\gamma=0.95$ e $S=\mathbb{R}$, significa que após a observações dos dados não estamos mais $100 \%$ seguros de que $x_{0}$ é um número real? No nosso entender os argumentos de ambos, Williams (1969) e Hoadley (1970), estão corretos sob o ponto de vista das distintas escolas de inferência estatística; clássica, no caso do primeiro autor e bayesiana, no caso do segundo autor. O que entra em causa aqui é o sentido do intervalo de confiança clássico e o significado do coeficiente de confiança. Concordamos com o questionamento de Hoadley (1970) a respeito do significado do coeficiente $\gamma$ e acreditamos que essa situação é um belo exemplo dos problemas que podemos ter na interpretação do coeficiente de confiança clássico.

Propostas mais recentes de soluções clássicas para o problema de calibração, incluindo propostas multivariadas, podem ser encontradas em Brown (1993). Entretanto, não vamos nos deter mais em soluções clássicas pois nosso trabalho foi elaborado sob o ponto de vista bayesiano. 


\subsection{Soluções bayesianas}

Como foi mencionado na seção anterior em um problema de calibração o objetivo é fazer inferência sobre o verdadeiro valor $x_{0}$ de uma variável $X$ conhecendo o valor $y_{0}$ de uma variável $Y$ relacionada com $X$. Essa situação pode ser pensada em termos multivariado considerado-se $X$ e $Y$ vetores de dimensões $p \times 1$ e $q \times 1$, respectivamente, com a restrição $p \geq q$ para evitar problemas de identificabilidade.

Com o objetivo de simplificar a notação, a partir de agora, não faremos distinção entre a quantidade aleatória $Y$ e a observação $y$, denotando ambos pela letra minúscula $y$. Além disso, $y$ denota um escalar e $\boldsymbol{y}$ um vetor. As letras maiúsculas, como por exemplo $\boldsymbol{A}, \boldsymbol{B}$ e $\boldsymbol{M}$, serão utilizadas para denotar matrizes.

$\mathrm{Na}$ visão bayesiana de inferência a solução do problema consiste em determinar uma distribuição a posteriori para o parâmetro desconhecido $\boldsymbol{x}_{0}$ dada a observação $\boldsymbol{y}_{0}$. Os elementos envolvidos nesse processo são:

(a) uma função densidade de probabilidades a priori (f.d.p.) para o vetor $\boldsymbol{x}_{\mathbf{0}}$, denotada por $p\left(\boldsymbol{x}_{0}\right)$;

(b) a função de verossimilhança de $\boldsymbol{x}_{0}$ dado $\boldsymbol{y}_{0}$, obtida a partir da especificação de uma família de distribuições de probabilidades para $\boldsymbol{y}_{\mathbf{0}}$ dado $\boldsymbol{x}_{\boldsymbol{0}}$.

A inferência ou atualização de sua opinião sobre $\boldsymbol{x}_{0}$, se produz via fórmula de Bayes como segue

$$
p\left(\boldsymbol{x}_{\mathbf{0}} \mid \boldsymbol{y}_{0}\right) \propto p\left(\boldsymbol{y}_{0} \mid \boldsymbol{x}_{0}\right) p\left(\boldsymbol{x}_{0}\right) .
$$

Considerando-se as suposições usuais:

(i) $\boldsymbol{y}_{0}=B^{t} \boldsymbol{x}_{0}+e_{0}$;

(ii) $\boldsymbol{B}, \boldsymbol{x}_{0}$ e $\boldsymbol{e}_{0}$ são independentes;

(iii) $E\left[e_{0} \mid \Sigma\right]=0, D\left[e_{0} \mid \Sigma\right]=\Sigma$, onde $\Sigma$ é uma matriz, $q \times q$, definida positiva (matriz de dispersão) independente de $\boldsymbol{x}_{0}$. Onde $E[\boldsymbol{w} \mid \theta]$ e $D[\boldsymbol{w} \mid \theta]$ denotam o vetor de esperança e a matriz de dispersão associados a um vetor $\boldsymbol{w}$. 
Temos que,

$$
p\left(\boldsymbol{y}_{0} \mid \boldsymbol{x}_{\mathbf{0}}\right)=\int_{\mathcal{M} x S_{q}^{+}} p\left(\boldsymbol{y}_{0} \mid \boldsymbol{B}, \boldsymbol{\Sigma}, \boldsymbol{x}_{\mathbf{0}}\right) p(\boldsymbol{B}, \boldsymbol{\Sigma}) d(\boldsymbol{B}, \boldsymbol{\Sigma})
$$

onde $p(\boldsymbol{B}, \boldsymbol{\Sigma})$ é uma densidade de probabilidade a priori para $(\boldsymbol{B}, \boldsymbol{\Sigma}), \mathcal{M}$ é o espaço das matrizes de ordem $p \times q$ e $S_{q}^{+}$é o espaço das matrizes definidas positivas de ordem $q \times q$. Por simplicidade de notação consideramos

$$
\int_{(\boldsymbol{B}, \boldsymbol{\Sigma})} h(\boldsymbol{\beta}, \boldsymbol{\Sigma}) d(\boldsymbol{B}, \boldsymbol{\Sigma})=\int_{\mathcal{M} x S_{q}^{+}} h(\boldsymbol{\beta}, \boldsymbol{\Sigma}) d(\boldsymbol{B}, \boldsymbol{\Sigma}) .
$$

Consequentemente,

$$
p\left(\boldsymbol{x}_{0} \mid \boldsymbol{y}_{0}\right) \propto p\left(\boldsymbol{x}_{\mathbf{0}}\right) \int_{(\boldsymbol{B}, \boldsymbol{\Sigma})} p\left(\boldsymbol{y}_{0} \mid \boldsymbol{B}, \boldsymbol{\Sigma}, \boldsymbol{x}_{\mathbf{0}}\right) p(\boldsymbol{B}, \boldsymbol{\Sigma}) d(\boldsymbol{B}, \boldsymbol{\Sigma}) .
$$

Diferentemente do caso clássico, inferências podem ser feitas utilizando-se uma observação e a informação a priori sobre os parâmetros $\boldsymbol{B}, \boldsymbol{\Sigma}$ que descrevem a relação entre as duas quantidades. Nesse sentido, a curva de calibração tem como objetivo ganhar informação a respeito das quantidades $\boldsymbol{B}$ e $\boldsymbol{\Sigma}$. Com este propósito são previamente fixados $n$ valores $\boldsymbol{X}^{t}=\left(\boldsymbol{x}_{1}, \boldsymbol{x}_{2}, \ldots, \boldsymbol{x}_{n}\right)$, e observadas as quantidade $\boldsymbol{Y}^{t}=\left(\boldsymbol{y}_{1}, \boldsymbol{y}_{2}, . ., \boldsymbol{y}_{n}\right)$, onde $\boldsymbol{x}_{i}$ e $\boldsymbol{y}_{i}$ são vetores de dimensão $p \times 1$ e $q \times 1$, respectivamente. Esse estágio é denominado experimento de calibração e esta informação é incorporada no cálculo da distribuição a posteriori, como segue.

$$
p\left(\boldsymbol{x}_{0} \mid \boldsymbol{y}_{\mathbf{0}}, \boldsymbol{Y}, \boldsymbol{X}\right) \propto p\left(\boldsymbol{y}_{\mathbf{0}}, \boldsymbol{Y} \mid \boldsymbol{x}_{0}, \boldsymbol{X}\right) p\left(\boldsymbol{x}_{0} \mid \boldsymbol{X}\right) .
$$

A solução de 2.7 depende das suposições feitas sobre o experimento de calibração. As seguintes suposições serão consideradas:

$(i v) \boldsymbol{y}_{i}=\boldsymbol{B} \boldsymbol{x}_{i}+e_{i}, \quad i=1,2, . ., n$

$(v)$ a independência condicional entre $\left(\boldsymbol{x}_{0}, \boldsymbol{y}_{0}\right)$ e $\boldsymbol{Y}$, dado $(\boldsymbol{B}, \boldsymbol{\Sigma}, \boldsymbol{X})$;

(vi) $E\left[e_{i} \mid \Sigma\right]=0, D\left[e_{i} \mid \Sigma\right]=\Sigma \quad i=1,2, \ldots n$. 
Temos que,

$$
\begin{gathered}
p\left(\boldsymbol{y}_{0}, \boldsymbol{Y} \mid \boldsymbol{x}_{\mathbf{0}}, \boldsymbol{X}\right)=\int_{(\boldsymbol{B}, \boldsymbol{\Sigma})} p\left(\boldsymbol{y}_{0}, \boldsymbol{Y} \mid \boldsymbol{x}_{0}, \boldsymbol{X}, \boldsymbol{B}, \boldsymbol{\Sigma}\right) p(\boldsymbol{B}, \boldsymbol{\Sigma}) d(\boldsymbol{B}, \boldsymbol{\Sigma})= \\
=\int_{(\boldsymbol{B}, \boldsymbol{\Sigma})} p\left(\boldsymbol{y}_{\mathbf{0}} \mid \boldsymbol{x}_{0}, \boldsymbol{B}, \boldsymbol{\Sigma}\right) p(\boldsymbol{Y} \mid \boldsymbol{X}, \boldsymbol{B}, \boldsymbol{\Sigma}) p(\boldsymbol{B}, \boldsymbol{\Sigma}) d(\boldsymbol{B}, \boldsymbol{\Sigma})= \\
=\int_{(\boldsymbol{B}, \boldsymbol{\Sigma})} p\left(\boldsymbol{y}_{\mathbf{0}} \mid \boldsymbol{x}_{0}, \boldsymbol{B}, \boldsymbol{\Sigma}\right) p(\boldsymbol{Y} \mid \boldsymbol{X}) p(\boldsymbol{B}, \boldsymbol{\Sigma} \mid \boldsymbol{X}, \boldsymbol{Y}) d(\boldsymbol{B}, \boldsymbol{\Sigma})= \\
=p(\boldsymbol{Y} \mid \boldsymbol{X}) \int_{(\boldsymbol{B}, \boldsymbol{\Sigma})} p\left(\boldsymbol{y}_{\mathbf{0}} \mid \boldsymbol{x}_{\mathbf{0}}, \boldsymbol{B}, \boldsymbol{\Sigma}\right) p(\boldsymbol{B}, \boldsymbol{\Sigma} \mid \boldsymbol{X}, \boldsymbol{Y}) d(\boldsymbol{B}, \boldsymbol{\Sigma}) .
\end{gathered}
$$

Consequentemente,

$$
p\left(\boldsymbol{x}_{0} \mid \boldsymbol{y}_{0}, \boldsymbol{Y}, \boldsymbol{X}\right) \propto p\left(\boldsymbol{x}_{0} \mid \boldsymbol{X}\right) \int_{(\boldsymbol{B}, \boldsymbol{\Sigma})} p\left(\boldsymbol{y}_{0} \mid \boldsymbol{B}, \boldsymbol{\Sigma}, \boldsymbol{x}_{\mathbf{0}}\right) p(\boldsymbol{B}, \boldsymbol{\Sigma} \mid \boldsymbol{Y},(\boldsymbol{X}) d(\boldsymbol{B}, \boldsymbol{\Sigma})
$$

Note que, a única diferença entre as expressões 2.8 e 2.6 é que a densidade dos parâmetros $\boldsymbol{B}, \boldsymbol{\Sigma}$ foi atualizada pelo experimento de calibração, isto é, $p(\boldsymbol{B}, \boldsymbol{\Sigma})$ é substituido por $p(\boldsymbol{B}, \boldsymbol{\Sigma} \mid \boldsymbol{Y}, \boldsymbol{x})$. Desta maneira, fica claro o papel do experimento de calibração.

$\mathrm{Na}$ análise Bayesiana paramétrica usual deve-se estabelecer uma distribuição a priori para os parâmetros, que expresse nossa incerteza sobre eles antes da observação dos dados experimentais. Algumas vezes essa opinião a priori é difícil de ser estabelecida, ou por não termos nenhum conhecimento a priori sobre a quantidade desconhecida de interesse, ou pela característica em si dos parâmetros em questão. A primeira situação é mais dificil de ocorrer quando esta quantidade é de fácil interpretação (parâmetro operacional), como por exemplo $\boldsymbol{x}$ e $\boldsymbol{y}$. A segunda situação refere-se a parâmetros de difícil interpretação ou parâmetros que não são as quantidades de interesse do problema mas que nos auxiliam a obter o resultado desejado, como por exemplo, $B$ e $\Sigma$. Uma solução para estas situações é considerar como distribuição a priori o que costuma-se denominar de "prioris não informativas" ou "prioris de referência". Esta segunda denominação nos parece mais adequada, já que o fato destas distribuições a priori serem realmente não informativas é 
bastante questionado, Bernardo and Smith (1994). O objetivo ao estabelecer-se este tipo de priori é influir o mínimo possível na análise inferencial a posteriori (após a observação dos resultados experimentais). Deste modo, pretende-se que toda a informação a respeito do parâmetro em questão seja proveniente das observações. É claro que, de fato isto nunca ocorre pois ao assumirmos um determinado modelo para os dados, o que é sempre feito na inferência paramétrica, estamos emitindo uma opinião a priori. Essas distribuições de referência são na maior parte das vezes impróprias, isto é, não são funções de densidades de probabilidade, por isso muitas vezes iremos nos referir a elas simplesmente como distribuições a priori impróprias. A utilização deste tipo de distribuições a priori é objeto de discussão entre os estatísticos bayesianos, ver O'Hagan (1994). Entre as criticas esta o argumento de incoerência, pelo fato de sua opinião a priori não estar sendo expressa em forma de uma distribuição de probabilidades. Outro problema é de como justificar o uso da fórmula de Bayes para a obtenção da distribuição a posteriori se não estamos lidando com distribuições de probabilidades. O defensores do seu uso, em geral, sustentam-se em argumentos pragmaticos e no fato dessas produzirem, algumas vezes, distribuições a posteriori próprias. Entretanto, seu uso só pode ser tolerado quando existir uma distribuição a posteriori própria.

Hoadley (1970) foi o primeiro a apresentar uma solução bayesiana para o problema de calibração controlada. O modelo proposto tem as suposições usuais da calibração linear univariada, isto é,

$$
y_{i}=\alpha+\beta x_{i}+e_{i}, e_{i}^{i . i . d .} N\left(0, \sigma^{2}\right), i=0,1,2, \ldots, n
$$

Os parâmetros do modelo são $\alpha, \beta, \sigma^{2}$ e $x_{0}$, onde os três primeiros são parâmetros perturbadores, já que nosso interesse é $x_{0}$. Hoadley (1970) considera para os parâmetros perturbadores $\left(\alpha, \beta, \sigma^{2}\right)$ uma distribuição a priori imprópria e para a quantidade de interesse $x_{0}$ uma densidade de probabilidades própria, genericamente denotada por $p\left(x_{0}\right)$. Assim, ele estabelece uma expressão para distribuição a posteriori de $x_{0}$, a qual será apresentada no próximo teorema. Considere a notação $\boldsymbol{x}=\left(x_{1}, x_{2}, \ldots, x_{n}\right)$, e $\boldsymbol{y}=\left(y_{1}, y_{2}, \ldots, y_{n}\right)$.

Teorema 2.1 Sob o modelo 2.9 suponha que a priori $x_{0} e\left(\alpha, \beta, \sigma^{2}\right)$ são independentes com densidades $p\left(x_{0} \mid \boldsymbol{x}\right)$ e $p\left(\alpha, \beta, \sigma^{2}\right)$, respectivamente. Se $p\left(x_{0} \mid \boldsymbol{x}\right)$ é própria $e$ $p\left(\alpha, \beta, \sigma^{2}\right) \propto 1 / \sigma^{2}$, então

$$
p\left(x_{0} \mid y_{0}, \boldsymbol{y}, \boldsymbol{x}\right) \propto p\left(x_{0} \mid \boldsymbol{x}\right) L\left(x_{0}\right)
$$


onde,

$$
\begin{gathered}
L\left(x_{0}\right)=\frac{\left(1+n+x_{0}^{2}\right)^{\frac{v}{2}}}{\left[1+n+R \hat{x}_{C}^{2}+\left(\frac{F}{v}+1\right)\left(x_{0}-R \hat{x}_{C}\right)^{2}\right]^{\frac{v+1}{2}}}, \quad \text { com } \\
v=n-2, \quad F=\frac{s_{x}^{2} \hat{\beta}^{2}}{S^{2}} \text { e } R=\frac{F}{(F+v)} .
\end{gathered}
$$

$\left[\left(x_{0}\right)\right.$ faz o papel de uma verossimilhança, com o problema de que $\int L(x) d x=\infty$. Como consequência deste fato não podemos considerar como distribuição a priori para $x_{0}$ a distribuição imprópria uniforme em $\mathbb{R}$, sob pena de não obtermos uma distribuição a posteriori própria. Hoadley (1970) considera uma particular distribuição a priori para $x_{0}$, na família das đistribuições $t$-Student, obtem que a distribuição a posteriori de $x_{0}$ também irá pertencer a esta família de distribuições, obtendo-se assim uma conjugação. Este é o resultado do próximo teorema.

Teorema 2.2 Sob as condiçôes do teorema 2.1 e supondo que

$$
x_{0} \mid x \sim T_{n-3}\left(\bar{x} ; s_{x}^{2} \frac{n+1}{n-3}\right), \quad n>3,
$$

então

$$
x_{0} \mid y_{0}, \boldsymbol{y}, \boldsymbol{x} \sim T_{n-2}\left(\hat{x}_{I} ; \frac{n+1+\frac{\left(\hat{x}_{I}\right)^{2}}{R}}{F+n-2}\right),
$$

onde $T_{n}\left(\mu, \sigma^{2}\right)$ denota a distribuição t-Student univariada não centrada com $n$ graus de liberdades e parâmetros de posição e dispersãa, $\mu$ e $\sigma^{2}$, respectivamente.

Uma consequência imediata do teorema 2.2 é que o estimador inverso, $\hat{X}_{I}$, é um estimador de Bayes, quando considera-se a perda quadrática . Estabelecida a distribuição a posteriori o problema de estimativa por intervalo fica trivialmente resolvido. Entretanto, observe que $\hat{X}_{I}$ é estimador de Bayes para esta particular distribuição (opinião) a priori. A distribuição a priori considerada no teorema 2.2 depende do planejamento do experimento de calibração, isto é, ela irá depender do tamanho da amostra $n$ e dos valores fixados $x_{i}^{\prime} s$, o que aparentemente é difícil de ser justificado. Como sua opinião a priori sobre o valor desconhecido de $x_{0}$ pode depender das condições do seu experimento de calibração? Tudo isso pode ser bastante questionável dentro de uma perspectiva subjetivista 
de probabilidade. Entretanto, invertendo um pouco a situação podemos dizer que se a nossa opinião a priori (que deve depender somente do nosso conhecimento a respeito do valor desconhecido $x_{0}$ ) está concentrada em torno de $\bar{x}$, na forma de uma distribuição $t$-Student como especificada no Teorema 2.2, então o estimador inverso é de Bayes. Além disso, a variância a priori é dada por

$$
\operatorname{Var}\left(x_{0} \mid \boldsymbol{x}\right)=s_{x}^{2} \frac{(n+1)}{(n-5)}, n>5
$$

de onde se conclui que, para $n$ grande, a variância de $x_{0}$ esta próxima de $s_{x}^{2}$, que é a variância amostral dos valores fixados $x_{i}^{\prime}$ s. É interessante observar que essa interpretação está de acordo com os estudos de simulação de Krutchkoff (1969) que indicam vantagens no estimador inverso para os casos onde $x_{0}$ esta dentro do domínio dos $x_{i}^{\prime} s$ e próximo de $\bar{x}$.

Posteriormente, Hunter and Lamboy (1981) também apresentaram uma solução bayesiana para o problema de calibração onde obtiveram expressões para a distribuição a posteriori. Eles consideram duas situações: quando a variância é conhecida e quando é desconhecida. Tais expressões são complicadas especialmente para o caso onde a variância é desconhecida que é a situação mais realística. No entanto, foram feitas algumas observações sobre o comportamento da distribuição a posteriori (através de um exemplo) supondo a variância conhecida. Observou-se que essa distribuição é bem aproximada pela distribuição normal centrada no estimador clássico (estimador de máxima verossimilhança) e que há uma coincidência muito grande entre as regiões de credibilidade bayesiana, usando a posteriori exata e a aproximação normal, e os intervalos de confiança clássicos de Fieller (1954).

Para obtenção da distribuição a posteriori desejada, Hunter and Lamboy (1981) consideram a seguinte reparametrização do problema de calibração

$$
\begin{aligned}
& y_{i}=\alpha+\beta x_{i}+e_{i}, \quad e_{i}^{i . i . d .} \sim N\left(0, \sigma^{2}\right) \\
& y_{0}=\eta+e_{0}, \quad e_{0} \sim N\left(0, \sigma^{2}\right), \quad e_{0} \text { independente de } e_{i}, \quad i=1, . ., n
\end{aligned}
$$

onde $\eta=\alpha+\beta x_{0}$. Eles derivam a distribuição a posteriori de $x_{0}$ a partir da distribuição a posteriori de $(\alpha, \beta)$ dado $\left(x_{1}, y_{1}\right), \ldots,\left(x_{n}, y_{n}\right)$ e da posteriori de $\eta$ dado $y_{0}$, considerando distribuições a prioris impróprias para $\alpha, \beta$ e $\eta$, e a independência entre $(\alpha, \beta)$ e $\eta$. $\mathrm{O}$ resultado é baseado na distribuição da razão de normais bivariadas, quando $\sigma^{2}$ é conhecido, e na razão de t-Student bivariada, quando $\sigma^{2}$ é desconhecido. Enquanto Hoadley (1970) 
utiliza uma distribuição a priori diretamente para o valor desconhecido de interesse $x_{0}$, Hunter and Lamboy (1981) utilizam uma distribuição a priori para o parâmetro $\eta$ que induz indiretamente uma distribuição a priori para $x_{0}$. Outra diferença entre as duas soluções bayesianas do problema de calibração é que Hunter and Lamboy (1981) supõem independência entre $\eta$ e $(\alpha, \beta)$, enquanto para Hoadley (1970) a independência é entre $x_{0}$ e $(\alpha, \beta)$. Vários autores, entre eles Hill (1981), afirmam que a independência assumida por Hoadley (1970) é mais natural.

Hill (1981), em discussão sobre o artigo de Hunter and Lamboy (1981), chama atenção para o fato de que esta solução é um caso particular da solução de Hoadley (1970), se considerarmos a seguinte distribuição a priori $p\left(\alpha, \beta, \sigma^{2}, x_{0}\right) \propto \frac{|\beta|}{\sigma^{2}}$. Segundo Hill (1981), um dos aspectos positivos do trabalho de Hunter and Lamboy (1981) é o fato de trazer uma justificativa bayesiana para o estimador clássico e as regiões de credibilidade de Fieller (1954), com a vantagem de não recair em coisas sem sentido como uma região $\mathbb{R}$ com confiança menor que 1 .

A extensão multivariada para o problema de calibração foi desenvolvida por Brown (1982) que obtem resultados tanto na perspectiva clássica quanto bayesiana. Na perspectiva bayesiana ele estende o trabalho de Hoadley, considerando o modelo

$$
\boldsymbol{y}_{i}=\boldsymbol{B}^{\boldsymbol{t}} \boldsymbol{x}_{i}+e_{i}, \quad e_{i} \stackrel{i . i . d .}{\sim} N_{q}(\mathbf{0} ; \boldsymbol{\Sigma}), \quad i=0,1,2, \ldots, n,
$$

onde $\boldsymbol{y}_{\boldsymbol{i}}$ e $\boldsymbol{x}_{\boldsymbol{i}}$ são vetores de dimensão $q \times 1$ e $p \times 1$, respectivamente, e $\boldsymbol{B}$ é uma matriz de dimensão $p \times q$. Por simplicidade de notação, estamos considerando os interceptos das retas iguais a zero. Para o caso mais geral, basta considerar $\boldsymbol{x}_{i}=\left(1, \boldsymbol{x}_{i}^{\prime}\right)$, onde $\boldsymbol{x}_{i}^{\prime}$ são os vetores originais, $i=0,1, . ., n$.

O resultado do próximo teorema foi obtido por Brown (1982) considerando a independência a priori entre $(\boldsymbol{B}, \Sigma)$ e $\boldsymbol{x}_{0}$ e o fato de $\boldsymbol{x}_{0}$ não depender de $\boldsymbol{x}_{1}, \ldots, \boldsymbol{x}_{n}$, justificado por estarmos trabalhando num problema de calibração controlada. Relembramos que $\boldsymbol{X}=\left(\boldsymbol{x}_{1}, \ldots, \boldsymbol{x}_{n}\right)^{t}$ e $\boldsymbol{Y}=\left(\boldsymbol{y}_{1}, . ., \boldsymbol{y}_{n}\right)^{t}$; e introduzimos a notação $\boldsymbol{X}_{*}=\left(\boldsymbol{x}_{0}, \boldsymbol{x}_{1}, . ., \boldsymbol{x}_{n}\right)$ e $Y_{*}=\left(\boldsymbol{y}_{0}, \boldsymbol{y}_{1}, \ldots, \boldsymbol{y}_{n}\right)$.

Teorema 2.3 Sob o modelo 2.13, suponha que a priori $\boldsymbol{x}_{0}$ e $(\boldsymbol{B}, \boldsymbol{\Sigma})$ são independentes com densidades $p\left(\boldsymbol{x}_{0}\right)$ e $p(\boldsymbol{B}, \boldsymbol{\Sigma})$, respectivamente. Se $p(\boldsymbol{B}, \boldsymbol{\Sigma}) \propto|\boldsymbol{\Sigma}|^{-\frac{(q+1)}{2}}$, então

$$
p\left(\boldsymbol{x}_{0} \mid \boldsymbol{y}_{0}, \boldsymbol{Y}, \boldsymbol{X}\right) \propto p\left(\boldsymbol{x}_{0}\right) L\left(x_{0}\right),
$$


onde

$$
L\left(\boldsymbol{x}_{0}\right)=\frac{\left(\sigma^{2}\left(\boldsymbol{x}_{0}\right)\right)^{\frac{v}{2}}}{\left[\sigma^{2}\left(\boldsymbol{x}_{0}\right)+\left(\boldsymbol{y}_{0}-\hat{\boldsymbol{B}}^{t} \boldsymbol{x}_{0}\right)^{t} \boldsymbol{S}^{-1}\left(\boldsymbol{y}_{0}-\hat{\boldsymbol{B}}^{t} \boldsymbol{x}_{0}\right)\right]^{\frac{(v+p)}{2}}},
$$

com $v=n+1-p-q$ e sendo

$$
\begin{aligned}
& \hat{\boldsymbol{B}}=\left(\boldsymbol{X}^{t} \boldsymbol{X}\right)^{-1} \boldsymbol{X}^{t} \boldsymbol{Y} \text { o e.m.v. de } \boldsymbol{B}, \\
& \boldsymbol{S}=(\boldsymbol{Y}-\boldsymbol{X} \hat{\boldsymbol{B}})^{t}(\boldsymbol{Y}-\boldsymbol{X} \hat{\boldsymbol{B}}) \text { a matriz dos resíduos, } e \\
& \sigma^{2}\left(\boldsymbol{x}_{\mathbf{0}}\right)=1+\boldsymbol{x}_{\mathbf{0}}{ }^{t}\left(\boldsymbol{X}^{t} \boldsymbol{X}\right)^{-1} \boldsymbol{x}_{\mathbf{0}} .
\end{aligned}
$$

$L\left(\boldsymbol{x}_{\mathbf{0}}\right)$ é integrável para $q>1$. Quando $q=1$ esta expressão coincide com a dada por Hoadley e como comentado anteriormente não é integrável.

Prova: Utilizando-se as propriedades usuais de probabilidade, temos que

$$
p\left(\boldsymbol{x}_{0} \mid \boldsymbol{y}_{0}, \boldsymbol{Y}, \boldsymbol{X}\right) \propto p\left(\boldsymbol{x}_{0} \mid \boldsymbol{X}\right) p\left(\boldsymbol{Y}_{*} \mid \boldsymbol{X}_{*}\right) .
$$

Mas, $p\left(\boldsymbol{Y}_{*} \mid \boldsymbol{X}_{*}\right)$ é a densidade preditiva ou marginal de $\boldsymbol{Y}_{*}$.

Sob a hipótese $p(\boldsymbol{B}, \boldsymbol{\Sigma}) \propto|\boldsymbol{\Sigma}|^{-\frac{(q+1)}{2}}$, temos que

$$
p\left(\boldsymbol{Y}_{*} \mid \boldsymbol{X}_{*}\right) \propto \int_{\boldsymbol{B}} \int_{\boldsymbol{\Sigma}}|\boldsymbol{\Sigma}|^{-\frac{n+1+q+1}{2}} e^{-\frac{1}{2} t r \boldsymbol{\Sigma}^{-1} \boldsymbol{A}} d \boldsymbol{\Sigma} d \boldsymbol{B}
$$

onde

$$
\boldsymbol{A}=\left(\boldsymbol{Y}_{*}-\boldsymbol{B} \boldsymbol{X}_{*}\right)^{t}\left(\boldsymbol{Y}_{*}-\boldsymbol{B} \boldsymbol{X}_{*}\right)
$$

Inicialmente vamos integrar 2.14 em relação a $\Sigma$. Para tal, observe que o integrando de 2.14 é o núcleo de uma distribuição Wishart (ver capítulo 4), portanto

$$
\int_{\Sigma}|\Sigma|^{-\frac{n+1+q+1}{2}} e^{-\frac{1}{2} t r \Sigma^{-1} A} d \Sigma \propto|A|^{\frac{n+1}{2}}
$$

Logo,

$$
p\left(\boldsymbol{Y}_{*} \mid \boldsymbol{X}_{*}\right) \propto \int_{\boldsymbol{B}}|\boldsymbol{A}|^{\frac{n+1}{2}} d \boldsymbol{B} \propto \int_{\boldsymbol{B}}\left|C_{3}+\left(B-C_{1}\right)^{t} C_{2}\left(B-C_{1}\right)\right|^{-\frac{n+1}{2}} d B
$$


onde

$$
\begin{aligned}
& C_{1}=\left[\hat{B}\left(\boldsymbol{X}^{t} \boldsymbol{X}\right)+\boldsymbol{y}_{0} \boldsymbol{x}_{0}^{t}\right]\left[\boldsymbol{X}^{t} \boldsymbol{X}+\boldsymbol{x}_{0} \boldsymbol{x}_{0}^{t}\right]^{-1}, \\
& C_{2}=\boldsymbol{X}^{t} \boldsymbol{X}+\boldsymbol{x}_{0} \boldsymbol{x}_{0}^{t} \quad \mathrm{e} \\
& C_{3}=S+\frac{\left(\boldsymbol{y}_{0}-\hat{\boldsymbol{B}} \boldsymbol{x}_{0}\right)\left(\boldsymbol{y}_{0}-\hat{\boldsymbol{B}} \boldsymbol{x}_{0}\right)^{t}}{1+\boldsymbol{x}_{0}^{t}\left(\boldsymbol{X}^{t} \boldsymbol{X}\right)^{-1} \boldsymbol{x}_{0}}
\end{aligned}
$$

Deste modo, o integrando de 2.15 pode ser identificado como o núcleo da densidade de uma matriz $t$-Student (ver capítulo 4), resultando que

$$
p\left(\boldsymbol{Y}_{*} \mid \boldsymbol{X}_{*}\right) \propto\left|\boldsymbol{C}_{2}\right|^{-\frac{p+1}{2}}\left|\boldsymbol{C}_{3}\right|^{-\frac{n+1-q}{2}} .
$$

Mas, podemos reescrever $\boldsymbol{C}_{2}$ e $\boldsymbol{C}_{3}$ como

$$
\begin{gathered}
C_{2}=\left|1+\boldsymbol{x}_{0}^{t}\left(\boldsymbol{X}^{t} \boldsymbol{X}\right)^{-1} \boldsymbol{x}_{0}\right|^{-\frac{(p+1)}{2}}\left|\boldsymbol{X}^{t} \boldsymbol{X}\right|^{-\frac{(p+1)}{2}} \\
C_{3}=\left|\nu+\frac{\left(\boldsymbol{y}_{0}-\hat{B} \boldsymbol{x}_{0}\right) \nu S^{-1}\left(\boldsymbol{y}_{0}-\hat{B} \boldsymbol{x}_{0}\right)^{t}}{1+\boldsymbol{x}_{0}^{t}\left(\boldsymbol{X}^{t} \boldsymbol{X}\right)^{-1} \boldsymbol{x}_{0}}\right|^{-\frac{(n+1-q)}{2}},
\end{gathered}
$$

onde $\nu=n-p-q$. Portanto,

$$
p\left(\boldsymbol{Y}_{*} \mid \boldsymbol{X}_{*}\right) \propto\left|\sigma^{2}\left(\boldsymbol{x}_{0}\right)\right|^{-\frac{(p+1)}{2}}\left|\frac{1+\left(\boldsymbol{y}_{0}-\hat{\boldsymbol{B}} \boldsymbol{x}_{0}\right)^{t} \boldsymbol{S}^{-1}\left(\boldsymbol{y}_{0}-\hat{\boldsymbol{B}} \boldsymbol{x}_{0}\right)}{\sigma^{2}\left(\boldsymbol{x}_{0}\right)}\right|^{-\frac{(n+1-p)}{2}} .
$$

Consequentemente,

$$
p\left(\boldsymbol{Y}_{*} \mid \boldsymbol{X}_{*}\right) \propto \frac{\left|\sigma^{2}\left(\boldsymbol{x}_{0}\right)\right|^{\frac{(n+1-p-q)}{2}}}{\left|\sigma^{2}\left(\boldsymbol{x}_{0}\right)+\left(\boldsymbol{y}_{0}-\hat{\boldsymbol{B}} \boldsymbol{x}_{0}\right)^{t} \boldsymbol{S}^{-1}\left(\boldsymbol{y}_{0}-\hat{\boldsymbol{B}} \boldsymbol{x}_{0}\right)\right|^{\frac{(n+1-q)}{2}}} .
$$

Recentemente, Kubokawa and Robert (1994) discutiram em termos da teoria da decisão a admissibilidade desses estimadores. Eles mostram que o estimador clássico não é admissível e que o estimador inverso é admissível para $p+q \geq 4$. A admissibilidade do estimador inverso provêm do fato dele ser um estimador de Bayes com risco finito. Eles propoẽm um estimador alternativo, que é Bayes quando utiliza-se a priori de referência de Bernardo (1979), que na sua contrução distingue os parâmetros de disturbio (nuisance) do parâmetro de interesse. Uma das vantagens desta distribuição a priori é que não se tem a dependência do tamanho da amostra, como na proposta por Brown. Entretanto, tal 
estimador não é obtido analiticamente mesmo tendo-se uma expressão analítica para a distribuição a posteriori. Para compará-lo com $\hat{X}_{C}$ e $\hat{X}_{I}$ eles fazem um estudo de simulação via Monte Carlo para os respectivos riscos. Numa situação particular, eles concluem que o estimador proposto é melhor que $\hat{X}_{I}$, em quase todo o espaço paramétrico. No entanto, a comparação com $\hat{X}_{C}$ é mais incerta, e parece que o método proposto tem vantagens para valores grandes de $\boldsymbol{x}_{0}$.

Outro trabalho recente em calibração bayesiana multivariada é o artigo de du Plessis and van der Merwe (1994), eles supõem a existência de um ponto $k$ no tempo a partir do qual há uma mudança na matriz de covariância. Esta solução é interessante para situações onde a suposição de homocedasticidade não é razoável, isto é, a variância não se mantem constante para os diversos valores de $x$. Os resultados obtidos também não são analíticos e a distibuição a posteriori é traçada com o auxílio de um algoritmo estocástico conhecido como amostrador de Gibbs(ver apêndice).

Observamos que mesmo sob as suposições de linearidade e normalidade tem-se dificuldades na obtenção de resultados analíticos no problema de calibração. Por isso, apesar da questão ser bastante antiga ainda desperta interesse, especialmente no campo multivariado. Alguns trabalhos com propostas clássicas tem sido desenvolvidos nesta campo, Brown and Sundberg (1987), Lieftinck-Koeijers (1987), Oman (1988), Mathew and Kasala (1994). Essas soluções são extremamente complicadas e acreditamos ser possível com a inferência bayesiana apresentar soluções mais simples para o problema de modo a permitir soluções também para situações onde as hipóteses de normalidade e linearidade não são satisfeitas.

No próximo capítulo buscamos soluções mantendo a suposição de linearidade e considerendo os erros na classe das distribuições elípticas, uma classe mais ampla do que a normal. 


\section{Capítulo 3}

\section{Calibração linear no modelo elíptico}

\subsection{Introdução}

No capítulo anterior apresentamos uma breve revisão da literatura para o problema de calibração controlada. Observamos que em todos esses procedimentos considera-se a suposição de normalidade para os erros. Um contexto interessante de ser estudado é o que ocorre quando a suposição de normalidade não é razoável. Por exemplo, como é sabido a distribuição normal é vulnerável a observações aberrantes (outliers). Várias técnicas estatísticas tem sido estudadas com o objetivo de diminuir este problema, denominadas técnicas robustas. Mesmo que em muitas delas a suposição de normalidade seja mantida, busca-se alternativas em técnica de contrução de estimadores que não sejam tão influenciados por estas observações aberrantes. Por outro lado, alguns autores propoẽm usar distribuições que apesar de preservarem a simetria da distribuição normal são mais resistentes a influência de dados aberrantes. Neste sentido, Lange, Little and Taylor (1989) sugerem um método de inferência robusta que consiste em substituir a suposição de normalidade pela distribuição t-Student. Como a distribuição t-Student possue caudas mais pesadas que a distribuição normal, ela permite a acomodação de valores discrepantes. Desse modo os outliers não são totalmente desconsiderados, como sugerem alguns métodos, mas entrariam na análise com um peso pequeno em relação às outras observações. Além disso, considerar a família de distribuições t-Student com $\nu$ graus de liberdades, $\nu \geq 1$, inclue também a distribuição normal se considerarmos o caso limite de $\nu \rightarrow \infty$. Portanto, o modelo normal estaria contemplado como um caso particular.

Como sugerido em Lange et al. (1989) o problema pode ser estendido para a família 
das distribuições elípticas. As distribuições elípticas formam uma classe generalizada de famílias paramétricas de distribuições probabilísticas que preservam a estrutura simétrica das distribuições normais, a família t-Student é um elemento dessa classe. Essa classe de distribuições tem despertado um crescente interesse nas últimas décadas com o objetivo de estudar a robustez dos procedimentos de inferência sob normalidade. Um trabalho recente que contem uma revisão detalhada das distribuições elípticas e suas propriedades é Arellano-Valle (1994).

Na seção 3.2 apresentamos uma breve revisão das distribuições elípticas, com alguns exemplos e as principais propriedades. Na seção 3.3 definimos o modelo de regressão linear elíptico, considerando-se dois casos: o modelo dependente e o modelo independente. Para o modelo elíptico dependente obtivemos, no teorema 3.1 , que a classe conjugada associada ao parâmetro de regressão $\beta$ é a classe das distribuições elípticas, supondo-se a variância conhecida. Além disso, a média a posteriori é uma combinação linear dos dados e não depende da especificação do modelo. Nas seções 3.4 e 3.5 tratamos do problema de calibração nos modelos elípticos dependentes e independentes, respectivamente. Finalmente, na seção 3.5 apresentamos uma aplicação, onde consideramos o modelo $t$-Student independente.

\subsection{Distribuições elípticas}

Neste capítulo vamos nos limitar a trabalhar com distribuições elípticas dominadas pela medida de Lesbegue, isto é, aquelas que possuem densidade.

Definição 3.1 Um vetor aleatório n-variado $\boldsymbol{y}$, absolutamente contínuo, tem distribuição elíptica com vetor de locação $\boldsymbol{\mu}$ e matriz de dispersão $\boldsymbol{\Sigma}$, se sua função densidade de probablidades é dada por

$$
f(\boldsymbol{y} \mid \boldsymbol{\mu}, \boldsymbol{\Sigma})=|\boldsymbol{\Sigma}|^{-\frac{1}{2}} g\left[(\boldsymbol{y}-\boldsymbol{\mu})^{t} \boldsymbol{\Sigma}^{-1}(\boldsymbol{y}-\boldsymbol{\mu})\right], \quad \boldsymbol{y} \in \mathbb{R}^{n}
$$

para alguma função $g$, tal que, $g(u) \geq 0$ para $u \geq 0$, $e$

$$
\int_{0}^{\infty} u^{\frac{n+1}{2}-1} g(u) d u=\frac{\Gamma\left(\frac{n+1}{2}\right)}{\pi^{\frac{n+1}{2}}} .
$$


A função $g$ é denominada função geradora da distribuição elíptica e a notação utilizada é $\boldsymbol{y} \sim E l_{n}(\boldsymbol{\mu}, \boldsymbol{\Sigma} ; g)$.

A seguir apresentamos alguns exemplos, de famílias de distribuições que pertencem a classe das distribuições elípticas.

Exemplo 3.1. Distribuição normal multivariada: considerando a função geradora

$$
g(u)=(2 \pi)^{\frac{-n}{2}} e^{\frac{-u}{2}}
$$

temos que,

$$
f(\boldsymbol{y} \mid \boldsymbol{\mu}, \boldsymbol{\Sigma})=|\boldsymbol{\Sigma}|^{-\frac{1}{2}}(2 \pi)^{\frac{-n}{2}} e^{\frac{-1}{2}(\boldsymbol{y}-\boldsymbol{\mu})^{\imath} \boldsymbol{\Sigma}^{-1}(\boldsymbol{y}-\boldsymbol{\mu})}, \quad \boldsymbol{y} \in \mathbb{R}^{n}
$$

que é a densidade de uma normal n-variada, denotada por $N_{n}(\boldsymbol{\mu}, \boldsymbol{\Sigma})$.

Exemplo 3.2. Distribuição t-multivariada : considerando a função geradora

$$
g(u)=\frac{\Gamma\left(\frac{(\nu+n)}{2}\right) \nu^{\frac{\nu}{2}}}{\Gamma\left(\frac{\nu}{2}\right) \pi^{\frac{n}{2}}}[\nu+u]^{\frac{-(\nu+n)}{2}}
$$

temos que, $\boldsymbol{y} \in \mathbb{R}^{n}, \nu \geq 0$

$$
f(\boldsymbol{y} \mid \boldsymbol{\mu}, \boldsymbol{\Sigma})=|\boldsymbol{\Sigma}|^{-\frac{1}{2}} \frac{\Gamma\left(\frac{(\nu+n)}{2}\right) \nu^{\frac{\nu}{2}}}{\Gamma\left(\frac{\nu}{2}\right) \pi^{\frac{n}{2}}}\left[\nu+(\boldsymbol{y}-\boldsymbol{\mu})^{t} \boldsymbol{\Sigma}^{-1}(\boldsymbol{y}-\boldsymbol{\mu})\right]^{\frac{-(\nu+n)}{2}}, \quad \boldsymbol{y} \in \mathbb{R}^{n}
$$

que é a densidade de uma t-Student n-variada $\operatorname{com} \nu$ graus de liberdades, denotada por $T_{n}(\boldsymbol{\mu}, \Sigma ; \nu)$.

Uma classe um pouco mais geral do que a t-Student é construída com a introdução de um novo parâmetro $\lambda$ e é denominada t-Student generalizada; sua densidade é dada por

$$
f(\boldsymbol{y} \mid \boldsymbol{\mu}, \boldsymbol{\Sigma})=|\boldsymbol{\Sigma}|^{-\frac{1}{2}} \frac{\Gamma\left(\frac{(\nu+n)}{2}\right) \lambda^{\frac{\nu}{2}}}{\Gamma\left(\frac{\nu}{2}\right) \pi^{\frac{n}{2}}}\left[\lambda+(\boldsymbol{y}-\boldsymbol{\mu})^{t} \boldsymbol{\Sigma}^{-1}(\boldsymbol{y}-\boldsymbol{\mu})\right]^{\frac{-(\nu+n)}{2}}
$$

e será denotada por $T_{n}(\boldsymbol{\mu}, \Sigma ; \lambda, \nu)$. Quando $\lambda=\nu$ temos a família da t-Student multivariada.

Exemplo 3.3. Distribuição normal contaminada: considerando a função geradora $g(u)=\xi g_{1}(u)+(1-\xi) g_{1}(\tau u) \quad, 0 \leq \xi \leq 1, \tau \geq 0$ onde $g_{1}$ é a função geradora de uma distribuição $N_{n}(\boldsymbol{\mu}, \boldsymbol{\Sigma})$. Então, temos que 


$$
f(\boldsymbol{y} \mid \boldsymbol{\mu}, \boldsymbol{\Sigma}, \xi, \tau)=\xi f_{1}(\boldsymbol{y} \mid \nu, \boldsymbol{\Sigma})+(1-\xi) f_{1}(\tau \boldsymbol{y} \mid \boldsymbol{\mu}, \boldsymbol{\Sigma})
$$

onde $f_{1}$ é a densidade de uma distribuição normal. Utilizaremos a notação $N C_{n}(\boldsymbol{\mu}, \boldsymbol{\Sigma} ; \xi, \tau)$.

Exemplo 3.4. Distribuição normal composta: considere a função geradora

$$
g(u)=\int_{0}^{\infty}(2 \pi v)^{\frac{-1}{2}} e^{-\frac{u}{2 v}} d G(v)
$$

onde $G$ é uma função de distribuição de probabilidades tal que, $G(0)=0$. Então, temos que

$$
f(\boldsymbol{y} \mid \boldsymbol{\mu}, \boldsymbol{\Sigma})=|\boldsymbol{\Sigma}|^{-\frac{1}{2}} \int_{0}^{\infty}(2 \pi v)^{\frac{-1}{2}} e^{-\frac{(\boldsymbol{y}-\boldsymbol{\mu})^{t} \boldsymbol{\Sigma}^{-1}(\boldsymbol{y}-\boldsymbol{\mu})}{2 v}} d G(v),
$$

que é a densidade de uma normal composta, também denominada de mistura de normais, cuja medida misturadora é $G$, e será denotada por $M N_{n}(\boldsymbol{\mu}, \Sigma ; G)$.

Exemplo 3.5. Distribuição de Pearson tipo II: considere a função geradora

$$
g(u)=\frac{\Gamma\left(\frac{n}{2}+m+1\right)}{\Gamma(m+1) \pi^{\frac{n}{2}}}(1-u)^{m}, \quad 0 \leq u \leq 1, m>-1
$$

temos que,

$$
f(\boldsymbol{y} \mid \boldsymbol{\mu}, \boldsymbol{\Sigma}, m)=|\boldsymbol{\Sigma}|^{-\frac{1}{2}} \frac{\Gamma\left(\frac{n}{2}+m+1\right)}{\Gamma(m+1) \pi^{\frac{n}{2}}}\left[1-(\boldsymbol{y}-\boldsymbol{\mu})^{t} \boldsymbol{\Sigma}^{-1}(\boldsymbol{y}-\boldsymbol{\mu})\right]^{m} .
$$

Exemplo 3.6. Distribuição logística: considere a função geradora

$$
g(u)=\frac{e^{-u}}{\left[1+e^{-u}\right]^{2}}, \quad u \geq 0
$$

temos que,

$$
f(\boldsymbol{y} \mid \boldsymbol{\mu}, \boldsymbol{\Sigma}, m)=|\boldsymbol{\Sigma}|^{-\frac{1}{2}} \frac{e^{-(\boldsymbol{y}-\boldsymbol{\mu})^{t} \boldsymbol{\Sigma}^{-1}(\boldsymbol{y}-\boldsymbol{\mu})}}{\left[1+e^{\left.-(\boldsymbol{y}-\boldsymbol{\mu})^{t} \boldsymbol{\Sigma}^{-1}(\boldsymbol{y}-\boldsymbol{\mu})\right]^{2}}\right.} .
$$




\section{- Principais Propriedades}

Considere $\boldsymbol{y} \sim E l_{n}(\boldsymbol{\mu}, \Sigma ; g)$, então as seguintes propriedades são válidas:

1) $O$ valor esperado e a matriz de covariâncias de $\boldsymbol{y}$, se existirem, são dados, respectivamente, por $E[\boldsymbol{y}]=\boldsymbol{\mu}$ e $V[\boldsymbol{y}]=\alpha_{g} \boldsymbol{\Sigma}$, onde $\alpha_{g}$ é uma constante positiva que depende da função geradora $g$.Por exemplo, no modelo Normal $\alpha_{g}=1$ e para o modelo t-Student $\operatorname{com} \nu$ graus de liberdades, $\alpha_{g}=\frac{\nu}{\nu-2}$, para $\nu>2$. No caso de $\Sigma=I_{n}, \alpha_{g}$ é a variância de cada distribuição marginal.

2) Se $\boldsymbol{z}=\boldsymbol{\mu}_{2}+\boldsymbol{\Sigma}_{2} \boldsymbol{y}$ então $\boldsymbol{z} \sim E l_{n}\left(\boldsymbol{\Sigma}_{2} \boldsymbol{\mu}+\boldsymbol{\mu}_{2}, \boldsymbol{\Sigma}_{2} \boldsymbol{\Sigma} \boldsymbol{\Sigma}_{2}^{t} ; g\right)$.

3) Suponha que $\boldsymbol{y}, \boldsymbol{\mu}$ e $\boldsymbol{\Sigma}$ são particionadas como segue

$$
\boldsymbol{y}=\left[\begin{array}{l}
\boldsymbol{y}_{A} \\
\boldsymbol{y}_{B}
\end{array}\right], \boldsymbol{\mu}=\left[\begin{array}{l}
\boldsymbol{\mu}_{A} \\
\boldsymbol{\mu}_{B}
\end{array}\right], \boldsymbol{\Sigma}=\left[\begin{array}{cc}
\boldsymbol{\Sigma}_{A 1} & \boldsymbol{\Sigma}_{A 2} \\
\boldsymbol{\Sigma}_{B 1} & \boldsymbol{\Sigma}_{B 2}
\end{array}\right]
$$

onde $\boldsymbol{y}_{A}$ e $\boldsymbol{\mu}_{A}$ são vetores $(m \times 1)$ e $\boldsymbol{\Sigma}_{A 1}$ é uma matriz $(m \times m), 1 \leq m<n$. Então,

$$
\boldsymbol{y}_{A} \sim E l\left(\boldsymbol{\mu}_{A}, \Sigma_{A 1} ; g^{*}\right), \quad \operatorname{com} g^{*}(u)=\frac{2 \pi^{\frac{m}{2}}}{\Gamma\left(\frac{m}{2}\right)} \int_{0}^{\infty} g\left(r^{2}+u\right) r^{m-1} d r, \quad u \geq 0
$$

e,

$$
\boldsymbol{y}_{A} \mid \boldsymbol{y}_{B} \sim E l\left(\boldsymbol{\mu}_{A}\left(\boldsymbol{y}_{B}\right), \boldsymbol{\Sigma}_{A 1 . B} ; g_{q\left(y_{B}\right)}\right),
$$

onde

$$
\begin{aligned}
& \boldsymbol{\mu}_{A}\left(\boldsymbol{y}_{B}\right)=\boldsymbol{\mu}_{A}+\boldsymbol{\Sigma}_{A 2} \Sigma_{B 2}^{-1}\left(\boldsymbol{y}_{B}-\boldsymbol{\mu}_{B}\right) \text { e } \quad \boldsymbol{\Sigma}_{A 1 . B}=\boldsymbol{\Sigma}_{A 1}-\boldsymbol{\Sigma}_{A 2} \boldsymbol{\Sigma}_{B 2}^{-1} \boldsymbol{\Sigma}_{B 1}, \\
& g_{a}(u)=\frac{g(u+a)}{\frac{\frac{m}{2}}{\Gamma\left(\frac{m}{2}\right)} \int_{0}^{\infty} g\left(r^{2}+a\right) r^{m-1} d r}, \quad u \geq 0, \quad q\left(\boldsymbol{y}_{B}\right)=\left(\boldsymbol{y}_{B}-\boldsymbol{\mu}_{B}\right)^{t} \boldsymbol{\Sigma}_{B 2}^{-1}\left(\boldsymbol{y}_{B}-\boldsymbol{\mu}_{B}\right) .
\end{aligned}
$$

4) Se $\boldsymbol{\Sigma}$ é diagonal, então $\boldsymbol{y}_{1}, \ldots, \boldsymbol{y}_{\boldsymbol{n}}$ são independentes se, e somente se, $\boldsymbol{y} \sim N_{n}(\boldsymbol{\mu}, \boldsymbol{\Sigma})$. 
Para mais detalhes e outras caracterizações na classe das distribuições elípticas ver Arellano-Valle (1994).

Exemplo 3.7. Seja $\boldsymbol{y} \sim T_{n}(\boldsymbol{\mu}, \boldsymbol{\Sigma} ; \lambda, \nu)$, então as distribuições marginais e condicionais são também t-Student generalizada. Considerando as partições de 3.3, temos que

$$
\boldsymbol{y}_{A} \sim T_{m}\left(\boldsymbol{\mu}_{A}, \boldsymbol{\Sigma}_{A 1} ; \lambda, \nu\right) \text { e } \boldsymbol{y}_{A} \mid \boldsymbol{y}_{B} \sim T_{m}\left(\boldsymbol{\mu}_{A}\left(\boldsymbol{y}_{B}\right), \boldsymbol{\Sigma}_{A 1 . B} ; \lambda_{q\left(\boldsymbol{y}_{B}\right)}, \nu_{m}\right),
$$
onde

$\nu_{m}=\nu+n-m, \lambda_{a}=\lambda+a \quad \mathrm{e}$

$\boldsymbol{\mu}_{A}\left(\boldsymbol{y}_{B}\right), \boldsymbol{\Sigma}_{A 1 . B}, q\left(\boldsymbol{y}_{B}\right)$ foram definidos em 3.4

Definição $3.2 U m$ vetor aleatório elíptico, $\boldsymbol{y} \sim E l_{n}(\boldsymbol{\mu}, \boldsymbol{\Sigma} ; g)$, é representável se

$$
g(u)=\int_{0}^{\infty}(2 \pi v)^{\frac{-1}{2}} e^{-\frac{u}{2 v}} d G(v),
$$

para alguma f.d. $G$, tal que $G(0)=0$.

A representabilidade de $g$ caracteriza uma subclasse das distribuições elípticas que será bastante útil no decorrer deste trabalho. Essa subclasse é a família das distribuições normais compostas (exemplo 3.4) ou mistura de normais.

Recentemente, Kano (1994) estabeleceu a equivalência entre a representabilidade de uma função geradora e o que ele chamou de consistência da família de distribuições. Essa propriedade de consistência refere-se a característica que algumas famílias de distribuições multivariadas possuem de que todas as suas densidades marginais estão na mesma família de distribuições, diferenciado-se da densidade conjunta original apenas por uma questão de dimensão. Mais precisamente, denotado-se por $g(u \mid n)$ a função geradora de um vetor $n$-dimensional, diremos que $g$ é consistente se

$$
\int_{-\infty}^{\infty} g\left(\sum_{j=1}^{n+1} x_{j}^{2} \mid n+1\right) d x_{n+1}=g\left(\sum_{j=1}^{n} x_{j}^{2} \mid n\right) .
$$

As distribuições normais, t-Student e normal contaminada possuem essa propriedade de consistência, enquanto que as distribuições logística e Pearson II não à possuem. 


\subsection{O modelo de regressão linear elíptico}

Considere o modelo de regressão linear

$$
\boldsymbol{y}=\boldsymbol{X} \boldsymbol{\beta}+e,
$$

onde $\boldsymbol{y}=\left(y_{1}, . ., y_{n}\right)^{t}$ é um vetor aleatório $n \times 1, \boldsymbol{X}=\left[x_{i j}\right]$ é uma matriz de dimensão $n \times k$ de constantes conhecidas, de posto $k<n, \boldsymbol{\beta}=\left(\beta_{1}, \ldots, \beta_{k}\right)^{t}$ é um vetor $k \times 1$ de parâmetros desconhecidos e $e=\left(e_{1}, . ., e_{n}\right)^{t}$ é um vetor de erros aleatórios.

De um modo geral serão consideradas as seguintes suposições a respeito dos erros: $E(\boldsymbol{e})=0$ e $\operatorname{Cov}(\boldsymbol{e})=\sigma^{2} \boldsymbol{I}_{\boldsymbol{n}}$; as quais são respeitadas se uma das duas hipóteses a seguir é acrescentada

(i) $\boldsymbol{e} \sim E l_{n}\left(\mathbf{0}, \sigma^{2} \boldsymbol{I}_{\boldsymbol{n}} ; g\right)$,

(ii) $e_{i} \stackrel{\text { ind }}{\sim} E l_{1}\left(0, \sigma^{2} ; g\right), \quad i=1,2, \ldots, n$.

As especificações $(i)$ e (ii) serão denominadas modelo elíptico dependente e modelo elíptico independente, respectivamente. Tais especificações têm sido consideradas por despertar interesse no estudo de robustez. No caso $(i)$ têm sido obtidos resultados interessantes tanto no contexto clássico como bayesiano. Zellner (1976) desenvolve um paralelo entre os contextos clássico e bayesiano sob o modelo $t$-dependente, isto é, supondo que $\boldsymbol{e} \sim T_{n}\left(0, \sigma^{2} \boldsymbol{I}_{n} ; \nu\right)$. No contexto clássico Zellner (1976) mostra que o estimador de máxima verossimilhança de $\boldsymbol{\beta}$ coincide com o respectivo estimador de máxima verossimilhança sob normalidade, $\hat{\boldsymbol{\beta}}=\left(\boldsymbol{X}^{t} \boldsymbol{X}\right)^{-1} \boldsymbol{X}^{t} \boldsymbol{y}$. Além disso, as inferências sob $\boldsymbol{\beta}$ baseadas tanto na estatística $t$ quanto na estatística $F$ são robustas (distribucionalmente robustas). No contexto bayesiano, Zellner (1976) mostra que considerando-se a distribuição a priori de referência usual para $\left(\boldsymbol{\beta}, \sigma^{2}\right)$, as inferências a posteriori sobre $\boldsymbol{\beta}$ são as mesmas que sob a suposição de normalidade. Entretanto, o mesmo não acontece com as inferências a posteriori sobre $\sigma^{2}$. Esses resultados tem sido estendidos para a classe geral das distribuições elípticas por diversos autores, ver por exemplo, Fang and Anderson (1990).

Lema 3.1 Sob o modelo de regressão linear elíptico dependente, se $p\left(\boldsymbol{\beta}, \sigma^{2}\right) \propto\left(1 / \sigma^{2}\right) p(\boldsymbol{\beta})$, então $p(\boldsymbol{\beta} \mid \boldsymbol{y})$ não depende da função geradora $g$.

Prova: ver Osiewalski and Steel (1993) 
No caso (ii) o tratamento analítico do modelo é em geral mais complicado. No contexto clássico a obtenção do estimador de máxima verossimilhança depende de algoritmos numéricos e as inferências são baseadas na teoria assintótica, ver por exemplo, Lange et al. (1989). No contexto bayesiano temos conhecimento do trabalho de Geweke (1993) que sob o modelo $t$-independente obtem a distribuição a posteriori aproximada para $\beta$ e $\sigma^{2}$ através do algoritmo numérico conhecido como amostrador de Gibbs (ver apêndice), considerando a distribuição a priori de referência usual para estes parâmetros. No problema de calibração não temos conhecimento de estudos considerando o modelo elíptico.

Os modelos $(i)$ e (ii) são equivalentes se e somente se, estivermos na família das distribuições normais, isto é, $g$ for a função geradora da distribuição normal.

Em inferência bayesiana muitas vezes é interessante considerar classes conjugadas de distribuições. Vamos denotar genericamente por $\theta$ o parâmetro de interesse e por $\boldsymbol{y} \mid \theta$ o modelo estatístico. Se as distribuições a priori e a posteriori de $\theta$ pertencem a uma mesma família de distribuições de probabilidades, $\mathcal{F}$, (de modo que elas irão diferir apenas em relação ao valor dos parâmetros que especificam essa distribuição) esta família é denominada classe conjugada ao modelo especificado para as observações, $\boldsymbol{y} \mid \theta$. Por exemplo, no modelo de regressão usual com erros normais a classe conjugada associada ao parâmetro $\beta$ é a família da distribuição normal. Neste sentido, vamos mostrar que para o problema de regressão linear simples, quando o parâmetro de interesse é $\boldsymbol{\beta}$, supondo-se $\sigma^{2}$ conhecida, a classe conjugada do modelo de regressão elíptico dependente é a classe das elípticas, a menos de algumas restrições sobre as funções geradoras do modelo e da distribuição a priori.

Teorema 3.1 Sejam $\boldsymbol{y} \mid \boldsymbol{\beta}, \sigma^{2} \sim E l_{n}\left(\boldsymbol{X} \boldsymbol{\beta}, \sigma^{2} \boldsymbol{I}_{\boldsymbol{n}} ; g\right)$ e $\boldsymbol{\beta} \mid \sigma^{2} \sim E l_{k}\left(\boldsymbol{b}_{0}, \sigma^{2} \boldsymbol{B}_{\mathbf{0}} ; h_{0}\right)$ tais que, $g$ e $h_{0}$ estão relacionadas através de uma função geradora $f$ como segue,

$$
\begin{array}{ll}
h_{0}(u)=\frac{\pi^{\frac{n}{2}}}{\Gamma\left(\frac{n}{2}\right)} \int_{0}^{\infty} r^{\frac{n-1}{2}} f(r+u) d r, & \\
g(u)=\frac{f(u+q(\boldsymbol{\beta}))}{h_{0}(q(\boldsymbol{\beta}))}, & q(\boldsymbol{\beta})=\frac{\left(\boldsymbol{\beta}-\boldsymbol{b}_{0}\right)^{\prime} \boldsymbol{B}_{0}^{-1}\left(\boldsymbol{\beta}-\boldsymbol{b}_{0}\right)}{\sigma^{2}} .
\end{array}
$$

Entâo

$$
\begin{aligned}
& \text { (i) } \boldsymbol{\beta} \mid \sigma^{2}, \boldsymbol{y} \sim E l_{k}\left(\boldsymbol{b}_{\mathbf{1}}, \sigma^{2} \boldsymbol{B}_{\mathbf{1}} ; h_{1}\right) \\
& \text { (ii) } \boldsymbol{y} \mid \sigma^{2} \sim E l_{n}\left(\boldsymbol{X} \boldsymbol{b}_{\mathbf{0}}, \sigma^{2}\left(\boldsymbol{I}_{n}+\boldsymbol{X} \boldsymbol{B}_{0} \boldsymbol{X}^{t}\right) ; g^{*}\right),
\end{aligned}
$$


onde

$$
\begin{gathered}
\boldsymbol{b}_{1}=\boldsymbol{b}_{0}+\boldsymbol{B}_{0} \boldsymbol{X}^{t} \boldsymbol{X}\left[\boldsymbol{I}_{k}-\boldsymbol{B}_{0}\left(\boldsymbol{I}_{k}+\boldsymbol{X}^{t} \boldsymbol{X}\right) \boldsymbol{B}_{0}\right]^{-1}\left(\hat{\boldsymbol{\beta}}-\boldsymbol{b}_{0}\right), \hat{\boldsymbol{\beta}}=\left(\boldsymbol{X}^{t} \boldsymbol{X}\right)^{-1} \boldsymbol{X} \boldsymbol{y}, \\
\boldsymbol{B}_{1}=\boldsymbol{B}_{0}-\boldsymbol{B}_{0} \boldsymbol{X}^{t}\left(\boldsymbol{I}_{n}+\boldsymbol{X} \boldsymbol{B}_{0} \boldsymbol{X}^{t}\right)^{-1} \boldsymbol{X} \boldsymbol{B}_{0}, \\
g^{*}(u)=\frac{\pi^{\frac{k}{2}}}{\Gamma\left(\frac{k}{2}\right)} \int_{0}^{\infty} r^{\frac{k-1}{2}} f(r+u) d r, u \geq 0 e \\
h_{1}(u)=\frac{f(u+q(\boldsymbol{y}))}{g^{*}(q(\boldsymbol{y}))}, \quad \operatorname{com} q(\boldsymbol{y})=\frac{\left(\boldsymbol{y}-\boldsymbol{X} \boldsymbol{b}_{0}\right)^{t}\left(\boldsymbol{I}_{n}+\boldsymbol{X} \boldsymbol{B}_{0} \boldsymbol{X}^{t}\right)^{-1}\left(\boldsymbol{y}-\boldsymbol{X} \boldsymbol{b}_{0}\right)}{\sigma^{2}} .
\end{gathered}
$$

\section{Prova :}

Vamos mostrar inicialmente que $(\boldsymbol{y}, \boldsymbol{\beta})$, condicionalmente a $\sigma^{2}$, tem uma distribuição conjunta elíptica $(n+k)$ variada e então, utilizando-se as propriedades das distribuições elípticas, verificamos os resultados $(i)$ e (ii).

$$
\begin{gathered}
p\left(\boldsymbol{y}, \boldsymbol{\beta} \mid \sigma^{2}\right)=p\left(\boldsymbol{y} \mid \boldsymbol{\beta}, \sigma^{2}\right) p\left(\boldsymbol{\beta} \mid \sigma^{2}\right)= \\
=\left(\sigma^{2}\right)^{-1} n^{-\frac{1}{2}}\left|\boldsymbol{B}_{0}\right|^{-\frac{1}{2}} g\left[\frac{(\boldsymbol{y}-\boldsymbol{X} \boldsymbol{\beta})^{t}(\boldsymbol{y}-\boldsymbol{X} \boldsymbol{\beta})}{\sigma^{2}}\right] h_{0}[q(\boldsymbol{\beta})] .
\end{gathered}
$$

Considerando as relações 3.7 , temos que

$$
p\left(\boldsymbol{y}, \boldsymbol{\beta} \mid \sigma^{2}\right) \propto f\left[\frac{(\boldsymbol{y}-\boldsymbol{X} \boldsymbol{\beta})^{t}(\boldsymbol{y}-\boldsymbol{X} \boldsymbol{\beta})+\left(\boldsymbol{\beta}-\boldsymbol{b}_{0}\right)^{t} \boldsymbol{B}_{0}^{-1}\left(\boldsymbol{\beta}-\boldsymbol{b}_{0}\right)}{\sigma^{2}}\right]
$$

Vamos denotar por

$$
\boldsymbol{Q}=(\boldsymbol{y}-\boldsymbol{X} \boldsymbol{\beta})^{t}(\boldsymbol{y}-\boldsymbol{X} \boldsymbol{\beta})+\left(\boldsymbol{\beta}-\boldsymbol{b}_{0}\right)^{t} \boldsymbol{B}_{0}^{-1}\left(\boldsymbol{\beta}-\boldsymbol{b}_{0}\right),
$$

então

$$
\begin{gathered}
Q=\left(\boldsymbol{y}-\boldsymbol{X} \boldsymbol{b}_{0}+\boldsymbol{X} \boldsymbol{b}_{0}-\boldsymbol{X} \boldsymbol{\beta}\right)^{t}\left(\boldsymbol{y}-\boldsymbol{X} \boldsymbol{b}_{0}+\boldsymbol{X} \boldsymbol{b}_{0}-\boldsymbol{X} \boldsymbol{\beta}\right)+\sigma^{2} q(\boldsymbol{\beta})= \\
=\left(\boldsymbol{y}-\boldsymbol{X} \boldsymbol{b}_{0}\right)^{t}\left(\boldsymbol{y}-\boldsymbol{X} \boldsymbol{b}_{0}\right)+\left(\boldsymbol{y}-\boldsymbol{X} \boldsymbol{b}_{0}\right)^{t}\left(\boldsymbol{X} \boldsymbol{b}_{0}-\boldsymbol{X} \boldsymbol{\beta}\right)+\left(\boldsymbol{X} \boldsymbol{b}_{0}-\boldsymbol{X} \boldsymbol{\beta}\right)^{t}\left(\boldsymbol{y}-\boldsymbol{X} \boldsymbol{b}_{0}\right)+
\end{gathered}
$$




$$
\begin{gathered}
+\left(\boldsymbol{X} \boldsymbol{b}_{0}-\boldsymbol{X} \boldsymbol{\beta}\right)^{t}\left(\boldsymbol{X} \boldsymbol{b}_{0}-\boldsymbol{X} \boldsymbol{\beta}\right)+\sigma^{2} q(\boldsymbol{\beta})= \\
=\left(\boldsymbol{y}-\boldsymbol{X} \boldsymbol{b}_{0}\right)^{t}\left(\boldsymbol{y}-\boldsymbol{X} \boldsymbol{b}_{0}\right)+2\left(\boldsymbol{y}-\boldsymbol{X} \boldsymbol{b}_{0}\right)^{t}\left(\boldsymbol{X} \boldsymbol{b}_{0}-\boldsymbol{X} \boldsymbol{\beta}\right)+\left(\boldsymbol{\beta}-\boldsymbol{b}_{0}\right)^{t}\left(\boldsymbol{X}^{t} \boldsymbol{X}\right)\left(\boldsymbol{\beta}-\boldsymbol{b}_{0}\right)+\sigma^{2} q(\boldsymbol{\beta})= \\
=\left(\boldsymbol{y}-\boldsymbol{X} \boldsymbol{b}_{0}\right)^{t}\left(\boldsymbol{y}-\boldsymbol{X} \boldsymbol{b}_{0}\right)+2\left(\boldsymbol{y}-\boldsymbol{X} \boldsymbol{b}_{0}\right)^{t}\left(\boldsymbol{X} \boldsymbol{b}_{0}-\boldsymbol{X} \boldsymbol{\beta}\right)+\left(\boldsymbol{\beta}-\boldsymbol{b}_{0}\right)^{t}\left[\left(\boldsymbol{X}^{t} \boldsymbol{X}\right)+\boldsymbol{B}_{0}^{-1}\right]\left(\boldsymbol{\beta}-\boldsymbol{b}_{0}\right)= \\
=\left(\begin{array}{c}
\boldsymbol{y}-\boldsymbol{X} \boldsymbol{b}_{0} \\
\boldsymbol{\beta}-\boldsymbol{b}_{0}
\end{array}\right)^{t}\left[\begin{array}{cc}
\boldsymbol{I}_{n} & \boldsymbol{X}^{t} \\
\boldsymbol{X} & \left(\boldsymbol{X}^{t} \boldsymbol{X}\right)+\boldsymbol{B}_{0}^{-1}
\end{array}\right]\left(\begin{array}{c}
\boldsymbol{y}-\boldsymbol{X} \boldsymbol{b}_{0} \\
\boldsymbol{\beta}-\boldsymbol{b}_{0}
\end{array}\right)= \\
=\left(\begin{array}{c}
\boldsymbol{y}-\boldsymbol{X} \boldsymbol{b}_{0} \\
\boldsymbol{\beta}-\boldsymbol{b}_{0}
\end{array}\right)^{t}\left[\begin{array}{cc}
\boldsymbol{I}_{n}+\boldsymbol{X}_{\boldsymbol{B}_{0} \boldsymbol{X}^{t}} & \boldsymbol{X} \boldsymbol{B}_{0} \\
\boldsymbol{B}_{0} \boldsymbol{X}^{t} & \boldsymbol{B}_{0}
\end{array}\right]^{-1}\left(\begin{array}{c}
\boldsymbol{y}-\boldsymbol{X} \boldsymbol{b}_{0} \\
\boldsymbol{\beta}-\boldsymbol{b}_{0}
\end{array}\right) .
\end{gathered}
$$

Consequentemente

$$
\left(\boldsymbol{y}, \boldsymbol{\beta} \mid \sigma^{2}\right) \sim E l_{n+k}\left(\boldsymbol{\mu}, \sigma^{2} \boldsymbol{\Sigma} ; f\right),
$$

onde

$$
\boldsymbol{\mu}=\left(\begin{array}{c}
\boldsymbol{X} \boldsymbol{b}_{0} \\
\boldsymbol{b}_{0}
\end{array}\right) \text { e } \boldsymbol{\Sigma}=\left[\begin{array}{cc}
\boldsymbol{I}_{n}+\boldsymbol{X} \boldsymbol{B}_{0} \boldsymbol{X}^{t} & \boldsymbol{X} \boldsymbol{B}_{0} \\
\boldsymbol{B}_{0} \boldsymbol{X}^{t} & \boldsymbol{B}_{0}
\end{array}\right]
$$

Utilizando-se a propriedade 3 das distribuições elípticas, obtemos que

$$
\boldsymbol{y} \mid \sigma^{2} \sim E l_{n}\left(\boldsymbol{X} \boldsymbol{b}_{0}, \boldsymbol{I}_{n}+\boldsymbol{X} \boldsymbol{B}_{0} \boldsymbol{X}^{t} ; g\right) \text { e } \boldsymbol{\beta} \mid \boldsymbol{y}, \sigma^{2} \sim E l_{k}\left(\boldsymbol{\mu}(\boldsymbol{y}), \boldsymbol{\Sigma}_{A 1 . B} ; g_{q(y)}\right)
$$

com

$$
\begin{gathered}
\boldsymbol{\mu}(\boldsymbol{y})=\boldsymbol{b}_{0}+\boldsymbol{B}_{0} \boldsymbol{X}^{t}\left[\boldsymbol{I}_{n}+\boldsymbol{X} \boldsymbol{B}_{0} \boldsymbol{X}^{t}\right]^{-1}\left(\boldsymbol{y}-\boldsymbol{X} \boldsymbol{b}_{0}\right) \\
\boldsymbol{\Sigma}_{A 1 . B}=\sigma^{2}\left[\boldsymbol{B}_{0}-\boldsymbol{B}_{0} \boldsymbol{X}^{t}\left[\boldsymbol{I}_{n}+\boldsymbol{X} \boldsymbol{B}_{0} \boldsymbol{X}^{t}\right]^{-1} \boldsymbol{X} \boldsymbol{B}_{0}\right] \mathrm{e} \\
q(\boldsymbol{y})=\left(\boldsymbol{y}-\boldsymbol{X} \boldsymbol{b}_{0}\right)^{t}\left[\boldsymbol{I}_{n}+\boldsymbol{X} \boldsymbol{B}_{0} \boldsymbol{X}^{t}\right]^{-1}\left(\boldsymbol{y}-\boldsymbol{X} \boldsymbol{b}_{0}\right)
\end{gathered}
$$


Manipulando um pouco obtemos $\boldsymbol{\mu}(\boldsymbol{y})=\boldsymbol{b}_{1}$. $\diamond$

O resultado (ii) do teorema 3.1 nos diz que, se a variância é conhecida, a distribuição preditiva também é elíptica. Este resultado será fundamental na obtenção da distribuição a posteriori do problema de calibração, apresentada no teorema 3.3., da próxima seção.

Em relação ao resultado $(i)$, observamos que após algumas simplificações $\boldsymbol{b}_{1}$ pode ser escrito como

$\boldsymbol{b}_{1}=\boldsymbol{B}_{0} \boldsymbol{X}^{t} \boldsymbol{X}\left[\boldsymbol{I}_{p}-\boldsymbol{B}_{0}\left(\boldsymbol{I}_{p}+\boldsymbol{X}^{t} \boldsymbol{X}\right) \boldsymbol{B}_{0}\right]^{-1} \hat{\boldsymbol{\beta}}+\left[\boldsymbol{I}_{n}-\boldsymbol{B}_{0} \boldsymbol{X}^{t} \boldsymbol{X}\left[\boldsymbol{I}_{p}-\boldsymbol{B}_{0}\left(\boldsymbol{I}_{p}+\boldsymbol{X}^{t} \boldsymbol{X}\right) \boldsymbol{B}_{0}\right]^{-1}\right] \boldsymbol{b}_{0}$

Portanto, $\boldsymbol{b}_{1}$ é uma combinação linear convexa de $\boldsymbol{b}_{0}$ ( a média a priori de $\boldsymbol{\beta}$ ) e de $\hat{\boldsymbol{\beta}}$ ( o estimador de máxima verossimilhança sob normalidade). Além disso, $\boldsymbol{b}_{1}$ não depende de $\sigma^{2}$ e nem da função geradora $f$. Resultando que, sob a perda quadrática, o estimador de Bayes para $\boldsymbol{\beta}$ é $b_{1}\left(E[\boldsymbol{\beta} \mid \boldsymbol{y}]=\boldsymbol{b}_{1}\right)$ independente do modelo especificado. Deste modo, a estimação pontual de $\boldsymbol{\beta}$ no modelo elíptico dependente será a mesma obtida sob normalidade. Observe que este resultado independe da distribuição a priori escolhida para $\sigma^{2}$. As diferenças em termos de estimação estão refletidas nas variâncias a posteriori, que serão dadas por

$$
V\left[\beta_{i} \mid \boldsymbol{y}, g\right]=E\left[V\left[\beta_{i} \mid \boldsymbol{y}, \sigma^{2}, g\right]\right]=\alpha_{h_{1}} b_{i i} E\left[\sigma^{2} \mid \boldsymbol{y}, g\right], \quad i=1,2, \ldots, k
$$

onde $\alpha_{h_{1}}=-2 \varphi_{h 1}^{\prime}(0)$ é a função característica associada a $h_{1}$ e a última esperança é calculada em relação à distribuição a posteriori de $\sigma^{2}$. A notação $E[X \mid g]$ indica que o modelo elíptico considerado tem função geradora $g$, analogamente para $V[\boldsymbol{X} \mid g]$. No caso particular do modelo normal substituimos $g$ por $\phi$. Como consequência deste resultado obtemos o seguinte corolário.

Corolário 3.1 Se $p\left(\sigma^{2}\right) \propto 1 / \sigma^{2}$ então

$$
E\left[\sigma^{2} \mid \boldsymbol{y}, f\right]=b_{11}^{-1} \frac{V\left[\beta_{1} \mid \boldsymbol{y}, \phi\right]}{\alpha_{h_{1}}} .
$$

Prova: Pelo Lema 1.1 sabemos que $V[\boldsymbol{\beta} \mid \boldsymbol{y}, g]$ não depende de $g$. Logo,

$$
V[\boldsymbol{\beta} \mid \boldsymbol{y}, g]=V[\boldsymbol{\beta} \mid \boldsymbol{y}, \phi]
$$

Considerendo-se a relação 3.8 obtemos o resultado desejado. 
O resultado deste corolário é bastante interessante pois nos permite obter uma expressão analítica para um estimador pontual da variância. Como já foi dito anteriormente, no modelo elíptico dependente a distribuição a posteriori de $\sigma^{2}$, utilizando-se uma distribuição a priori imprópria, depende da função geradora $g$ e pode ser extremamente difícil de obtê-la.

Exemplo 3.8. O modelo t-Student multivariado.

Considere $\boldsymbol{y} \mid \boldsymbol{\beta}, \sigma^{2} \sim T_{n}\left(\boldsymbol{X} \boldsymbol{\beta}, \sigma^{2} \boldsymbol{I}_{n} ; \nu+q(\boldsymbol{\beta}) ; \nu+k\right)$ e $\boldsymbol{\beta} \mid \sigma^{2} \sim T_{k}\left(\boldsymbol{b}_{0}, \sigma^{2} \boldsymbol{B}_{0} ; \nu\right)$. Neste caso, existe uma função geradora $f$ que satisfaz as condições 3.7 do teorema 3.1 , que é dada por

$$
f(u)=\frac{\Gamma\left(\frac{\nu+n+k}{2}\right) \nu^{\frac{\nu}{2}}}{\Gamma\left(\frac{\nu}{2}\right) \pi^{\frac{n+k}{2}}}[\nu+u]^{-\frac{\nu+n+k}{2}} .
$$

Para verificar isto devemos mostrar que

$$
g(u)=\frac{f(u+q(\boldsymbol{\beta}))}{h_{0}(q(\boldsymbol{\beta}))},
$$

onde $h_{0}$ é a função geradora de uma distribuição t-Student k-variada com $\nu$ graus de liberdades, e $g$ é a função geradora de uma distribuição t-Student generalizada n-variada com parâmetros $\nu+q(\boldsymbol{\beta})$ e $\nu+k$.

Utilizando-se a função geradora de uma distribuição t-Student generalizada, temos que

$$
h_{0}(q(\boldsymbol{\beta}))=\frac{\Gamma\left(\frac{\nu+k}{2}\right) \nu^{\frac{\nu}{2}}}{\Gamma\left(\frac{\nu}{2}\right) \pi^{\frac{k}{2}}}[\nu+q(\boldsymbol{\beta})]^{-\frac{\nu+k}{2}},
$$

mas

$$
f(u+q(\boldsymbol{\beta}))=\frac{\Gamma\left(\frac{\nu+k+n}{2}\right)}{\Gamma\left(\frac{\nu}{2}\right) \pi^{\frac{n+k}{2}}} \nu^{\frac{\nu}{2}}[\nu+q(\boldsymbol{\beta})+u]^{-\frac{\nu+k+n}{2}},
$$

portanto

$$
\frac{f(u+q(\boldsymbol{\beta}))}{h_{0}(q(\boldsymbol{\beta}))}=\frac{\Gamma\left(\frac{\nu+k+n}{2}\right)[\nu+q(\boldsymbol{\beta})]^{-\frac{\nu+k}{2}}}{\pi^{\frac{n}{2}} \Gamma\left(\frac{\nu+k}{2}\right)}[\nu+q(\boldsymbol{\beta})+u]^{-\frac{(\nu+k+n)}{2}}=g(u) .
$$

Logo, a relação 3.9 é verificada.

Aplicando-se o teorema 3.1 , obtemos que

$$
\boldsymbol{y} \mid \sigma^{2} \sim T_{n}\left(\boldsymbol{X} \boldsymbol{b}_{0}, \sigma^{2}\left(\boldsymbol{I}_{n}+\boldsymbol{X} \boldsymbol{B}_{0} \boldsymbol{X}^{t}\right) ; \nu\right) \text { e }
$$




$$
\boldsymbol{\beta} \mid \sigma^{2}, \boldsymbol{y} \sim T_{k}\left(\boldsymbol{b}_{1}, \sigma^{2} \boldsymbol{B}_{1} ; \lambda_{1}, r_{1}\right)
$$

onde $\lambda_{1}=\nu+q(\boldsymbol{y})$ e $\quad r_{1}=\nu+n$.

Nas próximas seções vamos estudar os modelos elípticos dependente e independente no contexto do problema de calibração.

\subsection{Calibração no modelo elíptico dependente}

Nesta seção vamos considerar o modelo elíptico dependente para estudar o problema de calibração linear. Por simplicidade e com o propósito de entender melhor as idéias principais, iniciamos com o modelo de calibração linear simples. Nesse contexto mostramos, em primeiro lugar, que considerando-se a distribuição a priori imprópria para o parâmetro $\sigma^{2}$, os resultados obtidos sob normalidade permanecem válidos. Este tipo de robustez é similar ao que ocorre para o parâmetro de regressão, cujo resultado foi apresentado no Lema 1.1. Logo a seguir, trabalhamos com a classe conjugada elíptica para o parâmetro $\boldsymbol{\beta}$ e uma distribuição a priori arbitrária para $\sigma^{2}$.

Considere o modelo de calibração linear simples

$$
y_{i}=\beta_{1}+\beta_{2} x_{i}+e_{i}, \quad i=0,1,2, . ., n,
$$

onde $x_{0}$ é uma quantidade desconhecida, mas $x_{1}, x_{2}, \ldots, x_{n}$ são quantidades conhecidas (previamente fixadas). Como antes, o parâmetro de interesse aqui é $x_{0}$ e portanto, $\beta_{1} \mathrm{e}$ $\beta_{2}$ são parâmetros perturbadores. $\mathrm{O}$ mesmo acontece com os parâmetros associados com a distribuição dos erros.

Com o objetivo de simplificar os resultados adotamos a notação $\boldsymbol{w}_{*}=\left(w_{0}, w_{1}, \ldots, w_{n}\right)$ para representar um vetor $n+1$ dimensional e $\boldsymbol{w}=\left(w_{1}, w_{2}, \ldots, w_{n}\right)$ para um vetor $n$ dimensional. Assim, $\boldsymbol{y}_{*}=\left(y_{0}, y_{1}, \ldots, y_{n}\right)^{t}, \boldsymbol{x}_{*}=\left(x_{0}, x_{1}, \ldots, x_{n}\right)^{t}$ e $\boldsymbol{X}_{*}=\left[\mathbf{1}_{n+1}, \boldsymbol{x}_{*}\right]$. onde $\mathbf{1}_{k}$ é um vetor de uns k-dimensional.

Desta forma, o modelo 3.10 pode ser escrito como

$$
\boldsymbol{y}_{*}=\boldsymbol{X}_{*} \boldsymbol{\beta}+e_{*},
$$

onde $\boldsymbol{\beta}=\left(\beta_{1}, \beta_{2}\right)^{t}$. 
Nesta seção vamos supor que $e_{*} \sim E l_{n}\left(0, \sigma^{2} \boldsymbol{I}_{n+1} ; g\right)$, isto é,

$$
p\left(e_{0}, . ., e_{n} \mid \sigma^{2}\right)=\left(\sigma^{2}\right)^{-\frac{(n+1)}{2}} g\left(\sum_{i=0}^{n} \frac{e_{i}^{2}}{\sigma^{2}}\right) .
$$

Consequentemente

$$
\boldsymbol{y}_{*} \mid \boldsymbol{X}_{*}, \boldsymbol{\beta}, \sigma^{2} \sim E l_{n+1}\left(\boldsymbol{X}_{*} \boldsymbol{\beta}, \sigma^{2} \boldsymbol{I}_{n+1} ; g\right),
$$

onde estamos supondo que $e_{*}$ é independente de $x_{0}$. Utilizando 3.11 e 3.12 temos que a função de verossimilhança do modelo elíptico dependente é dada por

$$
V_{\boldsymbol{y}_{*}}\left(\boldsymbol{\beta}, \sigma^{2}, x_{0}\right)=\left(\sigma^{2}\right)^{\frac{-(n+1)}{2}} g\left[\frac{\left(\boldsymbol{y}_{*}-\boldsymbol{X}_{*} \boldsymbol{\beta}\right)^{t}\left(\boldsymbol{y}_{*}-\boldsymbol{X}_{*} \boldsymbol{\beta}\right)}{\sigma^{2}}\right] .
$$

Note que 3.13 pode ser expressa em termos de

$$
\begin{gathered}
\hat{\boldsymbol{\beta}}_{*}=\left(\boldsymbol{X}_{*}{ }^{t} \boldsymbol{X}_{*}\right)^{-1} \boldsymbol{X}_{*}{ }^{t} \boldsymbol{y}_{*} \text { e } S_{*}^{2}=\frac{\left(\boldsymbol{y}_{*}-\boldsymbol{X}_{*} \hat{\boldsymbol{\beta}} \cdot\right)^{t}\left(\boldsymbol{y}_{*}-\boldsymbol{X}_{*} \hat{\boldsymbol{\beta}} *\right)}{(n-1)}, \text { uma vez que } \\
\left(\boldsymbol{y}_{*}-\boldsymbol{X}_{*} \boldsymbol{\beta}\right)^{t}\left(\boldsymbol{y}_{*}-\boldsymbol{X}_{*} \boldsymbol{\beta}\right)=\left(\boldsymbol{y}_{*}-\boldsymbol{X}_{*} \hat{\boldsymbol{\beta}}_{*}\right)^{t}\left(\boldsymbol{y}_{*}-\boldsymbol{X}_{*} \hat{\boldsymbol{\beta}}_{*}\right)+\left(\boldsymbol{\beta}-\hat{\boldsymbol{\beta}_{*}}\right)^{t}\left(\boldsymbol{X}_{*}{ }^{-1} \boldsymbol{X}_{*}\right)\left(\boldsymbol{\beta}-\hat{\boldsymbol{\beta}}_{*}\right)
\end{gathered}
$$

A seguir iremos considerar duas especificações para a distribuição a priori. A primeira considera uma distribuição a priori imprópria para $\sigma^{2}$ e a segunda considera a classe conjugada para $\boldsymbol{\beta}$.

Teorema 3.2 Sob o modelo de calibraçâo elíptico dependente, se $p\left(\boldsymbol{\beta}, \sigma^{2} \mid \boldsymbol{x}_{*}\right) \propto \frac{1}{\sigma^{2}} p(\boldsymbol{\beta})$ e $p\left(x_{0} \mid \boldsymbol{x}\right)$ é uma densidade própria, então

$$
p\left(x_{\mathbf{0}} \mid \boldsymbol{x}, \boldsymbol{y}_{*}\right) \propto p\left(x_{0} \mid \boldsymbol{x}\right) \int_{\boldsymbol{\beta}}\left(S_{*}^{2}\right)^{\frac{-(n+1)}{2}}\left[1+\frac{\left(\boldsymbol{\beta}-\hat{\boldsymbol{\beta}_{*}}\right)^{t} \boldsymbol{X}_{*}{ }^{t} \boldsymbol{X}_{*}\left(\boldsymbol{\beta}-\hat{\boldsymbol{\beta}_{*}}\right)}{(n-1) S_{*}^{2}}\right]^{-\frac{(n+1)}{2}} p(\boldsymbol{\beta}) d \boldsymbol{\beta}
$$

para qualquer função geradora da distribuição elíptica.

Prova: Sabemos que

$$
p\left(x_{0} \mid \boldsymbol{x}, \boldsymbol{y}_{*}\right) \propto p\left(\boldsymbol{y}_{*} \mid \boldsymbol{x}_{*}\right) p\left(x_{0} \mid \boldsymbol{x}\right) .
$$


Como $p\left(x_{0} \mid \boldsymbol{x}\right)$, a distribuição a priori de $x_{0}$, não depende do modelo proposto, a prova consiste em mostrar que $p\left(\boldsymbol{y}_{*} \mid \boldsymbol{x}_{*}\right)$ não depende da função geradora $g$.

Mas,

$$
p\left(\boldsymbol{y}_{*} \mid \boldsymbol{x}_{*}\right) \propto \int_{\left(\boldsymbol{\beta}, \sigma^{2}\right)} V_{\boldsymbol{y}_{*}}\left(\boldsymbol{\beta}, \sigma^{2}, x_{0}\right) p\left(\boldsymbol{\beta}, \sigma^{2} \mid \boldsymbol{x}_{*}\right) d \boldsymbol{\beta} d \sigma^{2} .
$$

De 3.13 e considerando a hipótese de priori imprópria para $\sigma^{2}$, temos que

$$
p\left(\boldsymbol{y}_{*} \mid \boldsymbol{x}_{*}\right) \propto \int_{\boldsymbol{\beta}} \int_{\sigma^{2}}\left(\sigma^{2}\right)^{\frac{-(n+1)}{2}} g\left[\frac{\left(\boldsymbol{y}_{*}-\boldsymbol{X}_{*} \boldsymbol{\beta}\right)^{t}\left(\boldsymbol{y}_{*}-\boldsymbol{X}_{*} \boldsymbol{\beta}\right)}{\sigma^{2}}\right] \frac{d \sigma^{2}}{\sigma^{2}} p(\boldsymbol{\beta}) d \boldsymbol{\beta} .
$$

Fazendo

$$
u=\frac{\left(\boldsymbol{y}_{*}-\boldsymbol{X}_{*} \boldsymbol{\beta}\right)^{t}\left(\boldsymbol{y}_{*}-\boldsymbol{X}_{*} \boldsymbol{\beta}\right)}{\sigma^{2}}
$$

obtemos para a integral mais interna, aplicando condição 3.2 , que

$$
\int_{u}\left[\frac{\left(\boldsymbol{y}_{*}-\boldsymbol{X}_{*} \boldsymbol{\beta}\right)^{t}\left(\boldsymbol{y}_{*}-\boldsymbol{X}_{*} \boldsymbol{\beta}\right)}{u}\right]^{\frac{-(n+1)}{2}} g(u) d u=\frac{\Gamma\left(\frac{n+1}{2}\right)}{\pi^{\frac{n+1}{2}}}\left[\left(\boldsymbol{y}_{*}-\boldsymbol{X}_{*} \boldsymbol{\beta}\right)^{t}\left(\boldsymbol{y}_{*}-\boldsymbol{X}_{*} \boldsymbol{\beta}\right)\right]^{\frac{-(n+1)}{2}},
$$

qualquer que seja a função geradora da distribuição elíptica. Finalmente, para obtermos a expressão 3.15 utilizamos a decomposição dada em 3.14 .

Portanto, a distribuição a posteriori de $x_{0}$ não depende da especificação do modelo e as inferências bayesianas sobre $x_{0}$ permanecem constantes sobre a classe das distribuições elípticas. Isso nos permite utilizar todos os resultados obtidos na literatura para o problema de calibração sob o modelo normal, mesmo que se esteja trabalhando com a hipótese de elipticidade conjunta.Deste modo, o próximo corolário é imediato do teorema e do resultado obtido por Hoadley (1970).

Corolário 3.2 Sob as condiçôes do teorema 3.2, se $p(\boldsymbol{\beta}) \propto C$ (constante) $e$ $x_{0} \mid \boldsymbol{x} \sim T_{1}\left(\bar{x}, s_{x}^{2}\left(\frac{n+1}{n-3}\right) ; n-3\right), n>3$, onde $s_{x}^{2}=\sum_{i=1}^{n} \frac{\left(x_{i}-\bar{x}\right)^{2}}{n}$ e $\bar{x}=\frac{1}{n} \sum_{i=1}^{n} x_{i}$, então

$$
x_{0} \mid \boldsymbol{x}, \boldsymbol{y}_{*} \sim T_{1}\left(\hat{x}_{I},\left[n+1+\frac{\left(1+\frac{n+1}{F}\right)\left(\hat{x}_{I}\right)^{2}}{F+n-2}\right] ; n-2\right),
$$

onde 


$$
\begin{aligned}
& \hat{x}_{I}=\bar{x}+\left(y_{0}-\bar{y}\right) \frac{s_{x}^{2} \hat{\beta}_{2}}{s_{y}^{2}} \text { é o estimador inverso para } x_{0}, \\
& \hat{\beta}_{2}=\frac{\sum_{i=1}^{n}\left(x_{i}-\bar{x}\right)\left(y_{i}-\bar{y}\right)}{n s_{x}^{2}} \text { é o estimador de máxima verossimilhança de } \beta_{2} e \\
& F=s_{x}^{2} \frac{\hat{\beta}_{2}^{2}}{S^{2}} \quad \operatorname{com} \quad S^{2}=\frac{(\boldsymbol{y}-\boldsymbol{X} \hat{\boldsymbol{\beta}})^{t}(\boldsymbol{y}-\boldsymbol{X} \hat{\boldsymbol{\beta}})}{n-2} .
\end{aligned}
$$

Assim, podemos concluir que o estimador inverso é um estimador de Bayes o qual é robusto na classe das distribuições elípticas. No entanto, salientamos que sua aplicação é restrita as suposições das distribuições a priori e todos os comentários feito no capítulo anterior sobre essa distribuição são mantidos. Relembramos que a suposição a priori sobre $x_{0}$ indica simetria em torno de $\bar{x}$ (média dos valores fixados para a calibração) e que $V\left(x_{0} \mid \boldsymbol{x}\right)=\frac{S_{x}^{2}(n+1)}{(n-5)}, n>5$.

Os resultados dados por Hunter and Lamboy (1981) e Kubokawa and Robert (1994) (ver capítulo1) sob suposição de normalidade continuam válidos na classe das distribuições elípticas dependentes, desde que seja considerada uma distribuição a priori imprópria para $\sigma^{2}$, do tipo $p\left(\sigma^{2}\right) \propto 1 / \sigma^{2}$.

Teorema 3.3 Sob o modelo de calibração elíptico dependente, $\operatorname{com}\left(\boldsymbol{\beta}, \sigma^{2}\right)$ independente de $x_{0}$ e $p\left(x_{0} \mid \boldsymbol{x}\right)$ uma densidade própria. Se $\boldsymbol{\beta} \mid \sigma^{2}, \boldsymbol{x} \sim E l_{2}\left(b_{0}, \sigma^{2} \boldsymbol{B}_{0}, h_{0}\right)$, onde $g$ e $h_{0}$ estão relacionadas como especificado em 3.7 , entâo

$$
p\left(x_{0} \mid \boldsymbol{x}, \boldsymbol{y}_{*}\right) \propto p\left(x_{0} \mid \boldsymbol{x}\right) \int_{0}^{\infty} e l_{n+1}\left(\boldsymbol{y}_{*} ; \boldsymbol{X}_{*} \boldsymbol{b}_{0}, \sigma^{2}\left(\boldsymbol{I}_{n+1}+\boldsymbol{X}_{*} \boldsymbol{B}_{0} \boldsymbol{X}_{*}{ }^{t}\right) ; g^{*}\right) p\left(\sigma^{2} \mid \boldsymbol{x}\right) d \sigma^{2},
$$

onde $l_{n}(z ; \mu, \Sigma ; g)$ denota a função densidade de probabilidade de uma elíptica n-variada com parâmetros de locação $\boldsymbol{\mu}$, matriz de dispersão $\boldsymbol{\Sigma}$ e função geradora $\mathrm{g}$.

Prova: A prova é imediata do resultado (ii) do teorema (3.1), basta considerar

$$
p\left(x_{0} \mid \boldsymbol{y}_{*}\right) \propto p\left(x_{0}\right) \int_{0}^{\infty} p\left(\boldsymbol{y}_{*} \mid x_{0}, \sigma^{2}\right) p\left(\sigma^{2}\right) d \sigma^{2}
$$

e observar que $\boldsymbol{y}_{*} \mid x_{0}, \sigma^{2}$ é a distribuição preditiva, quando $\sigma^{2}$ e $x_{0}$ são conhecidos, para $n+1$ observações.

A utilização deste resultado para obtenção de inferências sob $x_{0}$ depende da especificação do modelo, isto é, da forma fixada para as funções geradoras $g$ e $h_{0}$. 


\subsection{Calibração no modelo elíptico independente}

Nesta seção iremos trabalhar com o modelo de calibração linear simples definido em 3.10, sob o modelo elíptico independente. Suponha que

$$
e_{i} \mid \sigma^{2} \stackrel{i . i . d}{\sim} E l_{1}\left(0, \sigma^{2} ; g\right), \quad i=0,1, . ., n
$$

isto é,

$$
p\left(e_{0}, e_{1}, \ldots, e_{n} \mid \sigma^{2}\right)=\left(\sigma^{2}\right)^{-\frac{(n+1)}{2}} \prod_{i=0}^{n} g\left(\frac{e_{i}^{2}}{\sigma^{2}}\right) .
$$

Como estamos supondo que o vetor de erros $e_{*}=\left(e_{0}, . ., e_{n}\right)^{t}$ é independente de $\boldsymbol{x}_{*}=$ $\left(x_{0}, \ldots, x_{n}\right)^{t}$ e $\boldsymbol{\beta}=\left(\beta_{1}, \beta_{2}\right)^{t}$, de 3.10 segue que

$$
y_{i} \mid x_{i}, \boldsymbol{\beta}, \sigma^{2} \stackrel{i n d}{\sim} E l_{1}\left(\beta_{1}+\beta_{2} x_{i}, \sigma^{2} ; g\right) .
$$

Desta maneira a função de verossimilhança é dada por

$$
V_{\boldsymbol{y}_{*}}\left(\boldsymbol{\beta}, \sigma^{2}, x_{0}\right)=\left(\sigma^{2}\right)^{-\frac{(n+1)}{2}} \prod_{i=0}^{n} g\left[\frac{\left(y_{i}-\beta_{1}-\beta_{2} x_{i}\right)^{2}}{\sigma^{2}}\right] .
$$

Suponha que a função $g$ é representável. Neste caso o modelo apresentado em 3.17 pode ser considerado, de forma equivalente, em duas etapas:

(i) $y_{i} \mid \boldsymbol{\beta}, \sigma^{2}, x_{i}, \lambda_{i} \stackrel{i n d}{\sim} N\left(\beta_{1}+\beta_{2} x_{i} ; \lambda_{i} \sigma^{2}\right), \quad i=0,1, . ., n$, onde

(ii) $\lambda_{0}, \lambda_{1}, \ldots, \lambda_{n}$ são variáveis aleatórias não negativas i.i.d.com f.d.a G.

A condição de representabilidade de $g$ estabelece uma correspondência biunívoca entre a classe de funções $g$ representáveis e a classe das f.d. $G$ tais que $G(0)=0$. Deste modo, a análise bayesiana do problema de calibração sob o modelo elíptico independente é equivalente ao estudo deste problema num modelo normal heteroscedástico o qual é definido de forma hierárquica em $(i)$ e $(i i)$. Portanto, como veremos a seguir, uma vez definidas as distribuições a priori para $x_{0}, \boldsymbol{\beta}$ e $\sigma^{2}$, a etapa $(i)$ nos permite utilizar os resultados do modelo normal e sua inerente tratabilidade analítica. Para a etapa (ii) o problema se reduz a escolha de uma f.d.a. $G$ para os pesos $\lambda_{i}^{\prime} s$ e a um eventual problema de integração numérica. 
De fato, sob as condições $(i)$ e $(i i)$, a densidade preditiva $p\left(\boldsymbol{y}_{*} \mid \boldsymbol{x}_{*}\right)$ é dada por

$$
p\left(\boldsymbol{y}_{*} \mid \boldsymbol{x}_{*}\right)=\int_{\boldsymbol{\lambda}_{*}} p\left(\boldsymbol{y}_{*} \mid \boldsymbol{x}_{*}, \boldsymbol{\lambda}_{*}\right) d G_{*}\left(\boldsymbol{\lambda}_{*}\right),
$$

onde $\lambda_{*}=\left(\lambda_{0}, \lambda_{1}, \ldots, \lambda_{n}\right)^{t}, G_{*}\left(\boldsymbol{\lambda}_{*}\right)=\prod_{i=0}^{n} G\left(\lambda_{i}\right)$ e $p\left(\boldsymbol{y}_{*} \mid \boldsymbol{x}_{*}, \boldsymbol{\lambda}_{*}\right)$ é a densidade preditiva de um modelo normal heteroscedástico com pesos conhecidos $\lambda_{i}^{\prime} s$.

Considerando a densidade a priori $p\left(\boldsymbol{\beta}, \sigma^{2} \mid \boldsymbol{x}_{*}\right)$, temos que

$$
\begin{gathered}
p\left(\boldsymbol{y}_{*} \mid \boldsymbol{x}_{*}, \boldsymbol{\lambda}_{*}\right)=\int_{\left(\boldsymbol{\beta}, \sigma^{2}\right)} p\left(\boldsymbol{y}_{*} \mid \boldsymbol{\beta}, \sigma^{2}, \boldsymbol{x}_{*}, \boldsymbol{\lambda}_{*}\right) p\left(\boldsymbol{\beta}, \sigma^{2} \mid x_{*}\right) d\left(\boldsymbol{\beta}, \sigma^{2}\right)= \\
=\int_{\left(\boldsymbol{\beta}, \sigma^{2}\right)}\left|\boldsymbol{\Lambda}_{*}\right|^{\frac{-1}{2}}\left(2 \pi \sigma^{2}\right)^{\frac{-(n+1)}{2}} e^{-\frac{1}{2 \sigma^{2}}\left(\boldsymbol{y}_{*}-\boldsymbol{X}_{*} \boldsymbol{\beta}\right)^{t} \boldsymbol{\Lambda}_{*}\left(\boldsymbol{y}_{*}-\boldsymbol{X}_{*} \boldsymbol{\beta}\right)} p\left(\boldsymbol{\beta}, \sigma^{2} \mid \boldsymbol{x}_{*}\right) d\left(\boldsymbol{\beta}, \sigma^{2}\right),
\end{gathered}
$$

onde $\boldsymbol{\Lambda}_{*}=\operatorname{Diag}\left(\boldsymbol{\lambda}_{*}\right)$, com $\operatorname{Diag}(\boldsymbol{w})$ denotando a matriz diagonal cujos elementos da diagonal são os elementos do vetor $\boldsymbol{w}$ e $\boldsymbol{X}_{*}$ é como antes.

Note que, quando a distribuição a posteriori de $\boldsymbol{\beta}, \sigma^{2} \mid \boldsymbol{x}_{*}, \boldsymbol{y}_{*}, \boldsymbol{\lambda}_{*}$ é conhecida, podemos obter 3.20 a partir da relação

$$
p\left(\boldsymbol{y}_{*} \mid \boldsymbol{x}_{*}, \boldsymbol{\lambda}_{*}\right)=\frac{p\left(\boldsymbol{y}_{*} \mid \boldsymbol{\beta}, \sigma^{2}, \boldsymbol{x}_{*}, \boldsymbol{\lambda}_{*}\right) p\left(\boldsymbol{\beta}, \sigma^{2} \mid \boldsymbol{x}_{*}\right)}{p\left(\boldsymbol{\beta}, \sigma^{2} \mid \boldsymbol{x}_{*}, \boldsymbol{y}_{*}, \boldsymbol{\lambda}_{*}\right)} .
$$

No caso de $p\left(\boldsymbol{\beta}, \sigma^{2} \mid \boldsymbol{x}_{*}\right)$ ser uma distribuição imprópria, isto é, não ser uma função densidade de probabilidades, a distribuição a posteriori $p\left(x_{0} \mid \boldsymbol{x}, \boldsymbol{y}_{*}\right)$ pode também não ser uma função densidade de probabilidades. Neste caso devemos estudar condições sob as quais esta distribuição é própria.

A seguir vamos considerar duas particulares especificações para $p\left(\boldsymbol{\beta}, \sigma^{2} \mid \boldsymbol{x}_{*}\right)$, ambas motivadas pelo usual problema de regressão; a primeira considerando a usual priori de referência, que é imprópria, e a segunda a classe conjugada.

- Caso 1: A distribuição a priori de referência

Proposição 3.1 Seja $p\left(x_{0} \mid \boldsymbol{x}\right)$ uma densidade a priori própria e $p\left(\boldsymbol{\beta}, \sigma^{2} \mid \boldsymbol{x}_{*}\right) \propto 1 / \sigma^{2}$. Suponha que para cada $\left(\boldsymbol{x}_{*}, \boldsymbol{y}_{*}\right)$ existe $K\left(\boldsymbol{x}, \boldsymbol{y}_{*}\right)$ em $\mathbb{R}$ tal que

$$
E_{G \cdot}\left[q\left(\boldsymbol{\lambda}_{*} ; \boldsymbol{x}_{*}, \boldsymbol{y}_{*}\right)\right]<K\left(\boldsymbol{x}, \boldsymbol{y}_{*}\right)
$$


onde

$$
\begin{aligned}
& q\left(\boldsymbol{\lambda}_{*} ; \boldsymbol{x}_{*}, \boldsymbol{y}_{*}\right)=\left[S_{*}^{2}\left(\boldsymbol{\lambda}_{*}\right)\right]^{\frac{-(n-1)}{2}}\left|\boldsymbol{\Lambda}_{*}\left(\boldsymbol{X}_{*}{ }^{t} \boldsymbol{\Lambda}_{*}^{-1} \boldsymbol{X}_{*}\right)\right|^{\frac{-1}{2}}, \\
& (n-1) S_{*}^{2}\left(\boldsymbol{\lambda}_{*}\right)=\left[\boldsymbol{y}_{*}-\boldsymbol{X}_{*} \hat{\boldsymbol{\beta}}_{*}\left(\boldsymbol{\lambda}_{*}\right)\right]^{t} \boldsymbol{\Lambda}_{*}^{-1}\left[\boldsymbol{y}_{*}-\boldsymbol{X}_{*} \hat{\boldsymbol{\beta}}_{*}\left(\boldsymbol{\lambda}_{*}\right)\right] e \\
& \hat{\boldsymbol{\beta}}_{*}\left(\boldsymbol{\lambda}_{*}\right)=\left(\boldsymbol{X}_{*}{ }^{t} \boldsymbol{\Lambda}_{*}^{-1} \boldsymbol{X}_{*}\right)^{-1} \boldsymbol{X}_{*}{ }^{-1} \boldsymbol{\Lambda}_{*}^{-1} \boldsymbol{y}_{*} .
\end{aligned}
$$

Então $p\left(x_{0} \mid \boldsymbol{x}, \boldsymbol{y}_{*}\right)$ é uma densidade de probabilidades tal que,

$$
p\left(x_{0} \mid \boldsymbol{x}, \boldsymbol{y}_{*}\right) \propto p\left(x_{0} \mid \boldsymbol{x}\right) \int_{\boldsymbol{\lambda}_{*}} q\left(\boldsymbol{\lambda}_{*} ; \boldsymbol{x}_{*}, \boldsymbol{y}_{*}\right) d G_{*}\left(\boldsymbol{\lambda}_{*}\right) .
$$

Prova: De 3.16 e 3.20, segue que é necessário somente avaliar a integral

$$
\int_{\boldsymbol{\beta}, \sigma^{2}} p\left(\boldsymbol{y}_{*} \mid \boldsymbol{\beta}, \sigma^{2}, \boldsymbol{\lambda}_{*}, \boldsymbol{x}_{*}\right) \frac{1}{\sigma^{2}} d \sigma^{2} d \boldsymbol{\beta} .
$$

Integrando primeiro em relação a $\sigma^{2}$, obtemos

$$
\begin{gathered}
\int_{\sigma^{2}} p\left(\boldsymbol{y}_{*} \mid \boldsymbol{\beta}, \sigma^{2}, \boldsymbol{\lambda}_{*}, \boldsymbol{x}_{*}\right) d \sigma^{2}=\frac{\left|\boldsymbol{\Lambda}_{*}\right|^{\frac{-1}{2}}}{(2 \pi)^{\frac{n+1}{2}}} \int_{\sigma^{2}}\left(\sigma^{2}\right)^{\frac{-(n+1)}{2}} e^{\frac{-1}{2 \sigma^{2}}\left(\boldsymbol{y}_{*}-\boldsymbol{X} * \boldsymbol{\beta}\right)^{t} \boldsymbol{\Lambda}_{*}^{-1}\left(\boldsymbol{y}_{*}-\boldsymbol{X}_{*} \boldsymbol{\beta}\right)} \frac{d \sigma^{2}}{\sigma^{2}} \propto \\
\propto\left|\boldsymbol{\Lambda}_{*}\right|^{\frac{-1}{2}}\left[S_{*}^{2}\left(\boldsymbol{\lambda}_{*}\right)\right]^{\frac{-(n+1)}{2}}\left\{1+\frac{\left[\boldsymbol{\beta}-\hat{\boldsymbol{\beta}}_{*}\left(\boldsymbol{\lambda}_{*}\right)\right]^{t} \boldsymbol{X}_{*}{ }^{t} \boldsymbol{\Lambda}_{*}^{-1} \boldsymbol{X}_{*}\left[\boldsymbol{\beta}-\hat{\boldsymbol{\beta}}_{*}(\boldsymbol{\lambda} *)\right]}{(n-1) S_{*}^{2}\left(\boldsymbol{\lambda}_{*}\right)}\right\}^{\frac{-(n+1)}{2}} .
\end{gathered}
$$

A última expressão é o núcleo de uma densidade bivariada t-Student para $\boldsymbol{\beta}$, supondo conhecidos $\lambda_{*}$ e $\boldsymbol{x}_{*}$. Integrando esta em relação a $\boldsymbol{\beta}$ obtemos

$$
\left[S_{*}^{2}\left(\boldsymbol{\lambda}_{*}\right)\right]^{\frac{-(n-1)}{2}}\left|\boldsymbol{\Lambda}_{*}\left(\boldsymbol{X}_{*}{ }^{t} \boldsymbol{\Lambda}_{*}^{-1} \boldsymbol{X}_{*}\right)\right|^{\frac{-1}{2}}=q\left(\boldsymbol{\lambda}_{*} ; \boldsymbol{x}_{*}, \boldsymbol{y}_{*}\right)
$$

de modo que

$$
p\left(x_{0} \mid \boldsymbol{x}, \boldsymbol{y}_{*}\right) \propto p\left(x_{0} \mid \boldsymbol{x}\right) \int q\left(\boldsymbol{\lambda}_{*} ; \boldsymbol{x}_{*}, \boldsymbol{y}_{*}\right) d G_{*}\left(\boldsymbol{\lambda}_{*}\right)=p\left(x_{0} \mid \boldsymbol{x}\right) E_{G_{*}}\left(q\left(\boldsymbol{\lambda}_{*} ; \boldsymbol{x}_{*}, \boldsymbol{y}_{*}\right)\right) .
$$

A condição 3.22 garante que esta distribuição é própria e que

$$
\int_{x_{0}} p\left(x_{0} \mid \boldsymbol{x}\right) E_{G_{*}}\left(q\left(\boldsymbol{\lambda}_{*} ; \boldsymbol{x}_{*}, \boldsymbol{y}_{*}\right)\right) d x_{0}<K\left(\boldsymbol{x}, \boldsymbol{y}_{*}\right) . \diamond
$$


Geweke (1993) trabalhando com as mesmas condições a priori especificadas para a proposição 3.1. e sob o modelo de regressão linear t-independente, mostrou que a densidade a posteriori conjunta para $\boldsymbol{\beta}, \sigma^{2}$ é própria. Se o resultado de Geweke (1993) fosse válido não seria difícil verificar que a distribuição a posteriori para o problema de calibração é própria, sem a imposição da condição 3.22. Entretanto, há um erro neste resultado como veremos a seguir.

Como solução para a integral dada por 3.24, Geweke (1993)(pag.S24) obtem o seguinte resultado

$$
\left[S_{*}^{2}\left(\boldsymbol{\lambda}_{*}\right)\right]^{\frac{1}{2}}\left|\boldsymbol{\Lambda}_{*}\left(\boldsymbol{X}_{*}{ }^{t} \boldsymbol{\Lambda}_{*}^{-1} \boldsymbol{X}_{*}\right)\right|^{\frac{-1}{2}},
$$

onde deveria ter obtido o resultado de 3.25. Com base nesse resultado ele consegue um limite superior para a expressão 3.26 , concluindo que a integral 3.24 é finita.

- Caso 2: A distribuição a priori normal Gama-invertida (O’Hagan 1994). Sejam

$$
\begin{aligned}
& \boldsymbol{\beta} \mid \sigma^{2}, \boldsymbol{x}_{*} \sim N_{2}\left(\boldsymbol{b}_{0}, \sigma^{2} \boldsymbol{M}_{0}\right) e \\
& \sigma^{2} \mid \boldsymbol{x}_{*} \sim G I\left(\frac{n_{0}}{2}, \frac{r_{0}}{2}\right),
\end{aligned}
$$

onde $G I(a, b)$ representa a distribuição Gama invertida cuja função densidade é dada por

$$
f\left(\sigma^{2}\right)=\frac{r_{0}^{\frac{n_{0}}{2}}}{\Gamma\left(\frac{n_{0}}{2}\right)}\left(\sigma^{2}\right)^{-\frac{n_{0}}{2}} e^{-\frac{r_{0}}{2 \sigma^{2}}}
$$

Portanto,

$$
p\left(\boldsymbol{\beta}, \sigma^{2} \mid \boldsymbol{x}_{*}\right) \propto\left(\sigma^{2}\right)^{-\frac{n_{0}}{2}+1} e^{\frac{-1}{2 \sigma^{2}}\left[\left(\boldsymbol{\beta}-\boldsymbol{b}_{0}\right)^{t} \boldsymbol{M}_{0}^{-1}\left(\boldsymbol{\beta}-\boldsymbol{b}_{0}\right)+r_{0}\right]} .
$$

Utilizamos a notação $\left(\boldsymbol{\beta}, \sigma^{2}\right) \mid \boldsymbol{x}_{*} \sim N G I\left(\boldsymbol{b}_{0}, \boldsymbol{M}_{0}, n_{0}, r_{0}\right)$ para as especificações dadas em 3.27.

Na próxima proposição estabelecemos uma forma geral para a distribuição a posteriori de $x_{0}$ sob a especificação 3.27 .

Proposição 3.2 Sob o modelo 3.5, sejam $\left(\boldsymbol{\beta}, \sigma^{2}\right) \mid \boldsymbol{x}_{*} \sim N G I\left(\boldsymbol{b}_{0}, \boldsymbol{M}_{0}, n_{0}, r_{0}\right)$ e $p\left(x_{0} \mid \boldsymbol{x}\right)$ uma densidade a priori própria. Então

$$
p\left(x_{0} \mid \boldsymbol{x}, \boldsymbol{y}_{*}\right) \propto p\left(x_{0} \mid \boldsymbol{x}\right) \int_{\boldsymbol{\lambda}_{*}} t_{n+1}\left(\boldsymbol{y}_{*} ; \boldsymbol{X}_{*} \boldsymbol{b}_{0}, \frac{r_{0}}{n_{0}}\left(\boldsymbol{\Lambda}_{*}+\boldsymbol{X}_{*} \boldsymbol{M}_{0} \boldsymbol{X}_{*}{ }^{t}\right), n_{0}\right) d G_{*},\left(\boldsymbol{\lambda}_{*}\right)
$$


onde

$t_{p}(z ; \boldsymbol{\mu}, \boldsymbol{\Sigma}, d)$ denota a densidade de uma distribuiçâo t-Student p-variada com parâmetros de locação $\boldsymbol{\mu}$, matriz de dispersão $\boldsymbol{\Sigma}$ ed graus de liberdades.

Prova: Defina $Z_{*}=\Lambda_{*}^{-\frac{1}{2}} \boldsymbol{y}_{*}$ e $\boldsymbol{V}_{*}=\Lambda_{*}^{-\frac{1}{2}} \boldsymbol{X}_{*}$. Então,

$$
\boldsymbol{Z}_{*} \mid \boldsymbol{\beta}, \sigma^{2}, \boldsymbol{\lambda}_{*}, \boldsymbol{x}_{*} \sim N_{n+1}\left(\boldsymbol{V}_{*} \boldsymbol{\beta}, \sigma^{2} I_{n+1}\right) .
$$

Como $\left(\boldsymbol{\beta}, \sigma^{2}\right) \mid \boldsymbol{x}_{*} \sim N G I\left(\boldsymbol{b}_{0}, \boldsymbol{M}_{0}, n_{0}, r_{0}\right)$ temos, ver O'Hagan (1994) (cap.9,pag.258), que

$$
\boldsymbol{z}_{*} \mid \boldsymbol{\lambda}_{*}, \boldsymbol{x}_{*} \sim T_{n+1}\left(\boldsymbol{V}_{*} \boldsymbol{b}_{0}, \frac{r_{0}}{n_{0}}\left(\boldsymbol{I}_{n+1}+\boldsymbol{V}_{*} \boldsymbol{M}_{0} \boldsymbol{V}_{*}^{t}\right), n_{0}\right)
$$

Portanto,

$$
\boldsymbol{y}_{*} \mid \boldsymbol{\lambda}_{*}, \boldsymbol{x}_{*} \sim T_{n+1}\left(\boldsymbol{X}_{*} \boldsymbol{b}_{0}, \frac{r_{0}}{n_{0}}\left(\boldsymbol{\Lambda}_{*}+\boldsymbol{X}_{*} \boldsymbol{M}_{0} \boldsymbol{X}_{*}^{t}\right), n_{0}\right)
$$

Utilizando nossa notação, escrevemos

$$
p\left(\boldsymbol{y}_{*} \mid \boldsymbol{\lambda}_{*}, \boldsymbol{x}_{*}\right)=t_{n+1}\left(\boldsymbol{y}_{*} ; \boldsymbol{X}_{*} \boldsymbol{b}_{0}, \frac{r_{0}}{n_{0}}\left(\boldsymbol{\Lambda}_{*}+\boldsymbol{X}_{*} \boldsymbol{M}_{0} \boldsymbol{X}_{*}{ }^{t}\right), n_{0}\right),
$$

de onde segue que

$$
p\left(\boldsymbol{y}_{*} \mid \boldsymbol{x}_{*}\right)=\int_{\boldsymbol{\lambda}} t_{n+1}\left(\boldsymbol{y}_{*} ; \boldsymbol{X}_{*} \boldsymbol{b}_{0}, \frac{r_{0}}{n_{0}}\left(\boldsymbol{\Lambda}_{*}+\boldsymbol{X}_{*} \boldsymbol{M}_{0} \boldsymbol{X}_{*}{ }^{t}\right), n_{0}\right) d G_{*}\left(\boldsymbol{\lambda}_{*}\right) .
$$

Finalmente de 3.16 obtemos o resultado desejado. $\diamond$

No caso de $G_{*}$ ser degenerada no ponto $\boldsymbol{\lambda}_{*}=(1,1, . ., 1)$, temos o caso particular de erros normais e a distribuição a posteriori para $x_{0}$ pode ser escrita como

$$
p\left(x_{0} \mid \boldsymbol{x}, \boldsymbol{y}_{*}\right) \propto p\left(x_{0} \mid \boldsymbol{x}\right) t_{n+1}\left(\boldsymbol{y}_{*} ; \boldsymbol{X}_{*} \boldsymbol{b}_{0}, r_{0}\left(I_{n+1}+\boldsymbol{X}_{*} \boldsymbol{M}_{0} \boldsymbol{X}_{*}{ }^{t}\right), n_{0}\right) .
$$

No próximo corolário apresentamos uma versão analítica para esta distribuição a posteriori. Esse resultado é uma extensão do trabalho de Hoadley na medida em que admite uma classe de distribuições a priori próprias para os parâmetros $\left(\boldsymbol{\beta}, \sigma^{2}\right)$. 
Corolário 3.3 Sejam $e_{i} \mid \sigma^{2} \stackrel{\text { i.i.d. }}{\sim} N\left(0, \sigma^{2}\right)$ e $\left(\boldsymbol{\beta}, \sigma^{2}\right) \mid \boldsymbol{x} \sim N G I\left(\boldsymbol{b}_{0}, \boldsymbol{M}_{0}, n_{0}, r_{0}\right)$, $i=1,2, . ., n$. Então,

$$
p\left(x_{0} \mid \boldsymbol{x}, \boldsymbol{y}_{*}\right) \propto p\left(x_{0} \mid \boldsymbol{x}\right) L\left(x_{0}\right)
$$

onde

$$
L\left(x_{0}\right) \propto\left(1+\boldsymbol{x}_{0} \boldsymbol{M}_{2} \boldsymbol{x}_{0}{ }^{t}\right)^{\frac{-1}{2}}\left\{\lambda_{q(\boldsymbol{y})}+\frac{\left(y_{0}-\boldsymbol{x}_{0} \boldsymbol{M}_{1}\right)^{2}}{\left(1+\boldsymbol{x}_{0} \boldsymbol{M}_{2} \boldsymbol{x}_{0}{ }^{t}\right)}\right\}^{-\frac{\left(a_{0}+n+1\right)}{2}}
$$

$$
\begin{aligned}
& \text { com } \quad \boldsymbol{x}_{0}=\left(1, x_{0}\right) \quad \text { e } \quad \lambda_{q(y)}=n_{0}+\left(\boldsymbol{y}-\boldsymbol{X} \boldsymbol{b}_{0}\right)^{t}\left(\boldsymbol{I}_{n}+\boldsymbol{X} \boldsymbol{M}_{0} \boldsymbol{X}^{t}\right)^{-1}\left(\boldsymbol{y}-\boldsymbol{X} \boldsymbol{b}_{0}\right), \\
& M_{1}=\boldsymbol{b}_{0}+M_{0} \boldsymbol{X}^{t}\left(\boldsymbol{I}_{n}+\boldsymbol{X} M_{0} \boldsymbol{X}^{t}\right)^{-1}\left(\boldsymbol{y}-\boldsymbol{X} \boldsymbol{b}_{0}\right) e \\
& M_{2}=M_{0}-M_{0} \boldsymbol{X}^{t}\left(\boldsymbol{I}_{n}+\boldsymbol{X} M_{0} \boldsymbol{X}^{t}\right)^{-1} \boldsymbol{X} M_{0} .
\end{aligned}
$$

Prova: A equação 3.16 pode ser escrita como

$$
p\left(x_{0} \mid \boldsymbol{x}, \boldsymbol{y}_{*}\right) \propto p\left(x_{0} \mid \boldsymbol{x}\right) p\left(y_{0} \mid \boldsymbol{x}_{*}, \boldsymbol{y}\right) p\left(\boldsymbol{y} \mid \boldsymbol{x}_{*}\right)
$$

Mas, $p\left(\boldsymbol{y} \mid \boldsymbol{x}_{*}\right)=p(\boldsymbol{y} \mid \boldsymbol{x})$ não depende de $x_{0}$. Portanto,

$$
p\left(x_{0} \mid \boldsymbol{x}, \boldsymbol{y}_{*}\right) \propto p\left(x_{0} \mid \boldsymbol{x}\right) p\left(y_{0} \mid \boldsymbol{x}_{*}, \boldsymbol{y}\right) .
$$

Da Proposição 3.1 temos que $\boldsymbol{y}_{*} \mid \boldsymbol{x}_{*} \sim T_{n+1}\left(\boldsymbol{X}_{*} \boldsymbol{b}_{0}, \frac{r_{0}}{n_{0}}\left(\boldsymbol{I}_{n+1}+\boldsymbol{X}_{*} \boldsymbol{M}_{\mathbf{0}} \boldsymbol{X}_{*}{ }^{t}\right), n_{0}\right)$. Como $\boldsymbol{y}_{*}=\left(y_{0}, \boldsymbol{y}^{t}\right)^{t}$, das propriedades da distribuição t-Student generalizada (comentadas no exemplo 4) segue que a distribuição condicional de $y_{0}$ dado $\boldsymbol{y}, \boldsymbol{x}_{*}$ é também uma distribuição t-Student generalizada univariada. Assim, considerando-se as seguintes partições para o vetor de médias e a matriz de dispersão

$$
\boldsymbol{X}_{*} \boldsymbol{b}_{0}=\left(\begin{array}{c}
\boldsymbol{x}_{0} \boldsymbol{b}_{0} \\
\boldsymbol{X} \boldsymbol{b}_{0}
\end{array}\right), \quad \boldsymbol{I}_{n+1}+\boldsymbol{X}_{*} \boldsymbol{M}_{0} \boldsymbol{X}_{*}{ }^{t}=\left[\begin{array}{cc}
1+\boldsymbol{x}_{0} M_{0} \boldsymbol{x}_{0}{ }^{t} & \boldsymbol{x}_{0} \boldsymbol{M}_{0} \boldsymbol{X}^{t} \\
X M_{0} \boldsymbol{x}_{0}{ }^{t} & \boldsymbol{I}_{n}+\boldsymbol{X} M_{0} \boldsymbol{X}^{t}
\end{array}\right]
$$

obtemos

$$
y_{0} \mid \boldsymbol{x}_{*}, \boldsymbol{y} \sim T_{1}\left(\mu_{1}(\boldsymbol{y}), \boldsymbol{\Sigma}_{2} ; \lambda_{q(}(\boldsymbol{y}), \nu_{1}\right)
$$

onde

$$
\mu_{1}(\boldsymbol{y})=\boldsymbol{x}_{0} b_{0}+\boldsymbol{x}_{0} M_{0} \boldsymbol{X}^{t}\left(\boldsymbol{I}_{n}+\boldsymbol{X} M_{0} \boldsymbol{X}^{t}\right)^{-1}\left(\boldsymbol{y}-\boldsymbol{X} \boldsymbol{b}_{0}\right),
$$




$$
\begin{aligned}
& \boldsymbol{\Sigma}_{2}=1+\boldsymbol{x}_{0}\left[\boldsymbol{M}_{0}-\boldsymbol{M}_{0} \boldsymbol{X}^{t}\left(\boldsymbol{I}_{n}+\boldsymbol{X} \boldsymbol{M}_{0} \boldsymbol{X}^{t}\right)^{-1} \boldsymbol{X} \boldsymbol{M}_{0}\right] \boldsymbol{x}_{0}{ }^{t}, \\
& v_{1}=n_{0}+n \mathrm{e} \\
& \lambda_{q(\boldsymbol{y})}=n_{0}+\left(\boldsymbol{y}-\boldsymbol{X} \boldsymbol{B}_{0}\right)^{t}\left(\boldsymbol{I}_{n}+\boldsymbol{X} \boldsymbol{M}_{0} X^{t}\right)^{-1}\left(\boldsymbol{y}-\boldsymbol{X} \boldsymbol{B}_{0}\right) .
\end{aligned}
$$

Portanto,

$$
p\left(y_{0} \mid \boldsymbol{x}_{*}, \boldsymbol{y}\right) \propto L\left(x_{0}\right) .
$$

Finalmente, utilizando 3.31 obtemos o resultado desejado.

Exemplo 3.9. O modelo de calibração com erros normais contaminados.

Considere

$$
y_{i} \mid \boldsymbol{\beta}, \sigma^{2}, x_{i}, \tau, \xi \sim N C\left(\beta_{1}+\beta_{2} x_{i}, \sigma^{2} ; \tau, \xi\right), \quad i=0,1, . ., n .
$$

A primeira observação a ser feita é que a normal contaminada é representável e sua medida misturadora é dada por,

$$
G(\lambda)=\left\{\begin{array}{l}
\xi, \quad \text { se } \lambda=1 \\
1-\xi, \quad \text { se } \lambda=\tau .
\end{array}\right.
$$

Portanto, considerar a suposição de erros normais contaminados é equivalente à suposição de erros normais heterocedásticos com pesos $\lambda_{i}^{\prime} s$, onde os $\lambda_{i}^{\prime} s$ são i.i.d. com distribuição $G$. Neste caso, $G_{*}\left(\lambda_{*}\right)=\prod_{i=0}^{n} G\left(\lambda_{i}\right)=\xi^{r}(1-\xi)^{n+1-r}$, onde $r$ é o número de 1 's em $\lambda_{*}$. Utilizando-se a proposição 3.1 , temos que

$$
p\left(x_{0} \mid \boldsymbol{x}, \boldsymbol{y}_{*}\right) \propto p\left(x_{0} \mid \boldsymbol{x}\right) \sum_{r=0}^{n} \xi^{r}(1-\xi)^{n+1-r} t_{n+1}\left(\boldsymbol{y}_{*} ; \boldsymbol{X}_{*} \boldsymbol{b}_{0}, \frac{r_{0}}{n_{0}}\left(\boldsymbol{\Lambda}_{*}+\boldsymbol{X}_{*} \boldsymbol{M}_{0} \boldsymbol{X}_{*}{ }^{t}\right), n_{0}\right)
$$

Os resultandos das últimas duas proposições são interessantes pois mostram que a distribuição a posteriori de $x_{0}$ para o problema de calibração depende da forma da função geradora $g$ apenas através de $G_{*}$. Portanto, pesquisadores com distintos modelos e consequentemente distintas verossimilhanças, devem ter sua inferência sobre o valor desconhecido $x_{0}$ diferenciada apenas pelo valor esperado de uma particular função de $\boldsymbol{\lambda}_{\mathbf{*}}$, desde que tenham estabelecido as mesmas distribuições a priori.

Um problema com estes resultados é que em geral é difícil obter expressões analíticas fechadas da distribuição a posteriori de $x_{0}$, além do caso normal. Procedimentos do tipo 
Monte Carlo baseados em Cadeias de Markov (MCCM) têm sido muito utilizados nos últimos tempos para resolver este tipo de problema, Smith and Roberts (1993). Eles nos permitem obter uma estimativa bastante precisa da densidade desejada. Para a implementação do algoritmo conhecido como amostrador de Gibbs (ver apêndice) é necessário especificar todas as distribuições condicionais a posteriori de um parâmetro em relação aos demais. Vamos mostrar que sob certas especificações a priori as distribuições a posteriori condicionais são distribuições de probabilidades conhecidas. Para tal, observe que trabalhar com o modelo normal heteroscedástico especificado em 3.5 tem claras vantagens.

Proposição 3.3 Sob o modelo 3.5, sejam $\left(\boldsymbol{\beta}, \sigma^{2}\right) \mid \boldsymbol{x} \sim N G I\left(\boldsymbol{b}_{0}, \boldsymbol{\Sigma}_{0}, n_{0}, r_{0}\right)$ e $x_{0} \mid \boldsymbol{x} \sim$ $N\left(m_{0}, v_{0}\right), \operatorname{com}\left(\boldsymbol{\beta}, \sigma^{2}\right)$ independente de $x_{0}$. Então,

(i) $\boldsymbol{\beta} \mid \sigma^{2}, \boldsymbol{x}_{*}, \boldsymbol{\lambda}_{*}, \boldsymbol{y}_{*} \sim N_{2}\left(\boldsymbol{b}_{1}, \sigma^{2} \boldsymbol{\Sigma}_{1}\right)$,

onde

$\Sigma_{1}=\left(\Sigma^{-1}+\Sigma_{0}^{-1}\right)^{-1}, \quad b_{1}=\Sigma_{1}^{-1}\left(\Sigma_{0} b_{0}+\Sigma \hat{\beta}\right)$,

$\hat{\boldsymbol{\beta}}=\left(\hat{\beta}_{1}, \hat{\beta}_{2}\right)^{t}, \quad \boldsymbol{\Sigma}=\left[\begin{array}{cc}\hat{\lambda}+\frac{\hat{x}}{S_{x x}^{\lambda}} & \frac{-\hat{x}}{S_{x x}^{\lambda}} \\ \frac{-\hat{x}}{S_{x x}^{\lambda}} & \frac{1}{S_{x x}^{\lambda}}\end{array}\right] e$

$\hat{\beta}_{1}=\hat{y}-\hat{\beta}_{2} \hat{x} \quad, \quad \hat{\beta}_{2}=\frac{S_{x y}^{\lambda}}{S_{x x}^{\lambda}}$ são os estimadores de máxima verossimilhança.

$S_{x x}^{\lambda}=\sum_{i=0}^{n} \frac{\left(x_{i}-\hat{x}\right)^{2}}{\lambda_{i}}, S_{x y}^{\lambda}=\sum_{i=0}^{n} \frac{\left(x_{i}-\hat{x}\right)\left(y_{i}-\hat{y}\right)}{\lambda_{i}}$,

$\hat{x}=\left(\sum_{i=0}^{n} \frac{x_{i}}{\lambda_{i}}\right) \hat{\lambda}, \quad \hat{y}=\left(\sum_{i=0}^{n} \frac{y_{i}}{\lambda_{i}}\right) \hat{\lambda} e$

$\hat{\lambda}=\left(\sum_{i=0}^{n} \frac{1}{\lambda_{i}}\right)^{-1}$.

(ii) $\boldsymbol{\sigma}^{2} \mid \boldsymbol{\beta}, \boldsymbol{x}_{*}, \boldsymbol{\lambda}_{*}, \boldsymbol{y}_{*} \sim I G\left(n_{1}, r_{1}\right)$,

onde

$r_{1}=\sum_{i=0}^{n} \frac{\left[y_{i}-\beta_{1}-\beta_{2} x_{i}\right]^{2}}{\lambda_{i}}+r_{0} e n_{1}=n_{0}+n$.

(iii) $x_{0} \mid \boldsymbol{\beta}, \sigma^{2}, \boldsymbol{\lambda}_{*}, \boldsymbol{x}, \boldsymbol{y}_{*} \sim N\left(m_{1}, v_{1}\right)$,

onde

$m_{1}=v_{1}\left[\frac{m_{0}}{v_{0}}+\frac{\beta_{2}\left(y_{0}-\beta_{1}\right)}{\sigma^{2} \lambda_{0}}\right]$ e $v_{1}=\left[v_{0}^{-1}+\frac{\beta_{2}^{2}}{\sigma^{2} \lambda_{0}}\right]^{-1}$. 
Prova : (i) Vamos considerar a tranformação $\eta=\beta_{1}+\beta_{2} \hat{x}$ e mostrar que utilizando uma distribuição a priori imprópria para $\left(\eta, \beta_{2}\right),\left(\eta, \beta_{2}\right) \propto C$, as distribuições a posteriori de $\eta$ e $\beta_{2}$ são normais independentes. A função de verossimilhança associada ao problema, considerando o modelo normal heterocedástico é dada por

$$
V \boldsymbol{y}_{*}\left(\boldsymbol{\beta}, \sigma^{2}, \lambda_{*}, x_{0}\right) \propto\left(\sigma^{2}\right)^{\frac{-n}{2}}\left(\prod_{i=0}^{n} \lambda_{i}^{\frac{1}{2}}\right) e^{-\sum_{i=0}^{n} \frac{\left(y_{i}-\beta_{1}-\beta_{2} x_{i}\right)^{2}}{2 \sigma^{2} \lambda_{i}}} .
$$

Portanto,

$$
\begin{gathered}
p\left(\eta, \beta_{2} \mid \sigma^{2}, \boldsymbol{x}_{*}, \boldsymbol{\lambda}_{*}, \boldsymbol{y}_{*}\right) \propto e^{-\sum_{i=0}^{n} \frac{\left(y_{i}-\eta-\beta_{2}\left(x_{i}-\hat{x}\right)\right)^{2}}{2 \sigma^{2} \lambda_{i}}} \propto e^{-\frac{1}{2 \sigma^{2}} \sum_{i=0}^{n}\left(y_{i}^{2}-2 y_{i}\left(\eta+\beta_{2}\left(x_{i}-\hat{x}\right)\right)+\left(\eta+\beta_{2}\left(x_{i}-\hat{x}\right)\right)^{2} / \lambda_{i}\right.} \\
\propto e^{-\frac{1}{2 \sigma^{2}}\left[-2 \eta\left(\sum_{i=0}^{n} \frac{y_{i}}{\lambda_{i}}+\eta^{2} \sum_{i=0}^{n} \frac{1}{\lambda_{i}}-2 \beta_{2} \sum_{i=0}^{n} \frac{y_{i}\left(x_{i}-\hat{x}\right)^{2}}{\lambda_{i}}+2 \eta \beta_{2} \sum_{i=0}^{n} \frac{\left(x_{i}-\hat{x}\right)}{\lambda_{i}}+\beta_{2}^{2} \sum_{i=0}^{n} \frac{\left(x_{i}-\hat{x}\right)^{2}}{\lambda_{i}}\right]\right.} \propto \\
\propto e^{-\frac{1}{2 \sigma^{2}}\left[\sum_{i=0}^{n} \frac{1}{\lambda_{i}}(\eta-\hat{y})^{2}+\sum_{i=0}^{n} \frac{\left(x_{i}-\hat{x}\right)^{2}}{\lambda_{i}}\left(\beta_{2}-\hat{\beta}_{2}\right)^{2}\right]} .
\end{gathered}
$$

Logo, $\eta$ e $\beta_{2}$ são independentes com

$\eta \mid \sigma^{2}, \boldsymbol{x}_{*}, \boldsymbol{\lambda}_{*}, \boldsymbol{y}_{*} \sim N\left(\hat{y}, \sigma^{2}\left[\sum_{i=0}^{n} \frac{1}{\lambda_{i}}\right]^{-1}\right) \mathrm{e}$

$\beta_{2} \mid \sigma^{2}, \boldsymbol{x}_{*}, \boldsymbol{\lambda}_{*}, \boldsymbol{y}_{*} \sim N\left(\hat{\beta}_{2}, \frac{\sigma^{2}}{S_{\boldsymbol{x} x}^{\lambda}}\right)$.

Observe que considerar a distribuição a priori $\pi\left(\eta, \beta_{2}\right) \propto C$ implica que a distribuição a priori para $\left(\beta_{1}, \beta_{2}\right)$ também é uma distribuição imprópria, isto é, proporcional a uma constante. Além disso, do resultado obtido para $\left(\eta, \beta_{2}\right)$ não é difícil derivar, através das propriedades da esperança, variância e covariância de variáveis aleatórias, que

$$
\begin{aligned}
& E\left[\beta_{1} \mid \sigma^{2}, \boldsymbol{x}_{*}, \boldsymbol{\lambda}_{*}, \boldsymbol{y}_{*}\right]=\hat{y}-\frac{S_{x y}^{\lambda}}{S_{x x}^{\lambda}} \hat{x}, \\
& \operatorname{Var}\left[\beta_{1} \mid \sigma^{2}, \boldsymbol{x}_{*}, \boldsymbol{\lambda}_{*}, \boldsymbol{y}_{*}\right]=\sigma^{2}\left[\left(\sum_{i=0}^{n} \frac{1}{\lambda_{i}}\right)^{-1}+\frac{\hat{x}^{2}}{S_{\boldsymbol{x} x}^{\lambda}}\right] \mathrm{e} \\
& \operatorname{cov}\left[\beta_{1}, \beta_{2} \mid \sigma^{2}, \boldsymbol{x}_{*}, \boldsymbol{\lambda}_{*}, \boldsymbol{y}_{*}\right]=-\sigma^{2} \frac{\hat{x}}{S_{x x}^{\lambda}} .
\end{aligned}
$$

Deste modo, podemos dizer que sob a distribuição a priori imprópria,

$$
\left(\beta_{1}, \beta_{2}\right) \mid \sigma^{2}, \boldsymbol{x}_{*}, \boldsymbol{\lambda}_{*}, \boldsymbol{y}_{*} \sim N_{2}(\hat{\boldsymbol{\beta}}, \boldsymbol{\Sigma})
$$


A próxima etapa é considerar a distribuição a priori $N_{2}\left(b_{0}, \sigma^{2} \Sigma_{0}\right)$ para $\beta=\left(\beta_{1}, \beta_{2}\right)$. Vamos denotar por $p_{\pi}\left(\boldsymbol{\beta} \mid \sigma^{2}, \boldsymbol{x}_{*}, \boldsymbol{\lambda}_{*}, \boldsymbol{y}_{*}\right)$ a distribuição a posteriori condicional, quando considerada a distribuição a priori normal bivariada, e por $p_{C}\left(\boldsymbol{\beta} \mid \sigma^{2}, \boldsymbol{x}_{*}, \boldsymbol{\lambda}_{*}, \boldsymbol{y}_{*}\right)$ a distribuição a posteriori condicional considerando a distribuição a priori imprópria. Então, podemos escrever

$$
p_{\pi}\left(\boldsymbol{\beta} \mid \sigma^{2}, \boldsymbol{x}_{*}, \boldsymbol{\lambda}_{*}, \boldsymbol{y}_{*}\right) \propto \pi(\boldsymbol{\beta}) p_{C}\left(\boldsymbol{\beta} \mid \sigma^{2}, \boldsymbol{x}_{*}, \boldsymbol{\lambda}_{*}, \boldsymbol{y}_{*}\right)
$$

utilizando as simplificações usuais da classe conjugada normal obtemos o resultado desejado.

Os resultados (ii) e (iii) são obtidos facilmente se considerada a fórmula de bayes, as funções densidades a priori para $\left(\boldsymbol{\beta}, \sigma^{2}\right)$ e $x_{0}$, e a expressão para verossimilhança dada em 3.33 .

A seguir apresentamos dois exemplos onde uma distribuição $G$ é especificada de modo a obtermos os modelos $t$-Student e normal contaminado.

Exemplo 3.10. Considere o modelo de calibração com erros normais contaminados, especificado no exemplo 3.9. Para a implementação do amostrador de Gibbs, além das distribuições condicionais obtidas na proposição 3.3., devemos estabelecer a distribuição $p\left(\boldsymbol{\lambda}_{*} \mid \boldsymbol{\beta}, \sigma^{2}, \boldsymbol{x}_{*}, \boldsymbol{y}_{*}\right)$, supondo conhecidos $\tau$ e $\xi$. Considerando-se a função de verossimilhança 3.33 e $G_{*}$, temos que

$$
\begin{gathered}
p\left(\boldsymbol{\lambda}_{*} \mid \boldsymbol{\beta}, \sigma^{2}, \boldsymbol{x}_{*}, \boldsymbol{y}_{*}\right) \propto \prod_{i=0}^{n} G\left(\lambda_{i}\right) \lambda_{i}^{-\frac{1}{2}} e^{-\frac{\left(y_{i}-\beta_{1}-\beta_{2} x_{i}\right)^{2}}{2 \sigma^{2} \lambda_{i}}}= \\
=\prod_{i=0}^{n}\left[\xi e^{-\frac{\left(y_{i}-\beta_{1}-\beta_{2} x_{i}\right)^{2}}{2 \sigma^{2}}} \boldsymbol{I}_{\left(\lambda_{i}=1\right)}+(1-\xi) \tau^{-\frac{1}{2}} e^{-\frac{\left(y_{i}-\beta_{1}-\beta_{2} x_{i}\right)^{2}}{2 \sigma^{2} \tau}} \boldsymbol{I}_{\left(\lambda_{i}=\tau\right)}\right] .
\end{gathered}
$$

Nesta caso os $\lambda_{i}^{\prime} s$ são independentes com distribuição

$$
p\left(\lambda_{i} \mid \boldsymbol{\beta}, \sigma^{2}, \boldsymbol{x}_{*}, \boldsymbol{y}_{*}\right)=\frac{\xi \phi\left(\frac{y_{i}-\beta_{1}-\beta_{2} x_{i}}{\sigma}\right)}{K^{\sigma}} \boldsymbol{I}_{\left(\lambda_{i}=1\right)}+\frac{(1-\xi) \phi\left(\frac{y_{i}-\beta_{1}-\beta_{2} x_{i}}{\tau \sigma}\right)}{K} \boldsymbol{I}_{\left(\lambda_{i}=\tau\right)}
$$

onde $\phi$ é a densidade da distribuição normal padrão e

$$
K=\xi \phi\left(\frac{y_{i}-\beta_{1}-\beta_{2} x_{i}}{\sigma}\right)+(1-\xi) \phi\left(\frac{y_{i}-\beta_{1}-\beta_{2} x_{i}}{\tau \sigma}\right) .
$$


Exemplo 3.11. O modelo de calibração com erros $t$-Student.

Considere

$$
y_{i} \mid \boldsymbol{\beta}, \sigma^{2}, x_{i}, \nu \sim T\left(\beta_{1}+\beta_{2} x_{i}, \sigma^{2} ; \nu\right) .
$$

Então, $f\left(y_{i} \mid \boldsymbol{\beta}, \sigma^{2}, \nu\right)$ é representável e sua mediada misturadora $G$ é uma $I G\left(\frac{\nu}{2}, \frac{\nu}{2}\right)$. De fato, da f.d.p. da gama-invertida derivamos a seguinte igualdade

$$
\int_{0}^{\infty} s^{-a-1} e^{\frac{-b}{s}} d s=\Gamma(a) b^{-a} .
$$

Considerando $a=\frac{(\nu+n)}{2}, b=\frac{(u+\nu)}{2}$ e a função geradora da distribuição t-Student (ver exemplo 3.2 ), temos que

$$
g(u)=\frac{\nu^{\frac{\nu}{2}}}{\Gamma\left(\frac{\nu}{2}\right) \pi^{\frac{n}{2}}} \int_{0}^{\infty} s^{-\frac{(\nu+n)}{2}-1} e^{\frac{-(u+\nu)}{2 s}} d s
$$

que é equivalente a

$$
g(u)=\int_{0}^{\infty}(\pi s)^{\frac{-n}{2}} e^{\frac{-u}{2 s}} f(s) d s
$$

Onde $f(s)=\frac{\nu \frac{\nu}{2}}{\Gamma\left(\frac{\nu}{2}\right)} s^{\frac{-\nu}{2}-1} e^{\frac{-\nu}{2 s}}$ é a função densidade de uma gama inversa . Portanto,

$$
g(u)=\int_{0}^{\infty}(\pi s)^{\frac{-n}{2}} e^{\frac{-u}{2 s}} d G(s)
$$

onde $G$ é uma $I G\left(\frac{\nu}{2}, \frac{\nu}{2}\right)$.

Assim, considerar a suposição de erros $t$-Student independentes é equivalente à suposição, de forma hierárquica, de erros normais heteroscedásticos, com a seguinte distribuição a priori para os pesos $\lambda_{i}^{\prime} s, \frac{\nu}{\lambda_{i}} \sim \chi_{\nu}^{2}$ (qui-quadrado com $\nu$ graus de liberdades). Esta observação é feita em Geweke (1993) para o problema de estimação dos parâmetros de regressão. Para a implementação do amostrador de Gibbs no modelo t-Student devemos utilizar os resultados da proposição 3.3 e especificar a distribuição condicional a posteriori de $\lambda_{*}$, que será dada por

$$
p\left(\boldsymbol{\lambda}_{*} \mid \boldsymbol{\beta}, \sigma^{2}, \boldsymbol{x}_{*}, \boldsymbol{y}_{*}\right)=\prod_{i=0}^{n} p\left(\lambda_{i} \mid \boldsymbol{\beta}, \sigma^{2}, \boldsymbol{x}_{*}, \boldsymbol{y}_{*}\right),
$$


onde

$\lambda_{i} \mid \boldsymbol{\beta}, \sigma^{2}, \boldsymbol{x}_{*}, \boldsymbol{y}_{*} \sim I G\left(\frac{\nu+1}{2}, \frac{S_{i}}{2}\right), \quad S_{i}=\frac{\left(y_{i}-\beta_{1}-\beta_{2} x_{i}\right)^{2}}{\sigma^{2}}+\nu, i=0,1, . ., n$.

Uma discussão específica a respeito do modelo $t$-Student independente é apresentada em Branco, Bofarine and Iglesias (1996), onde são feitos alguns estudos de simulação e apresentada a aplicação que será discutida na próxima seção.

\subsection{Medindo o comprimento de uma maratona}

Nesta aplicação reanalizamos o conjunto de dados estudado por Smith and Corbett (1987). Um percurso utilizado para uma prova de maratona é subdividido em 25 trechos, incluindo trechos cujos comprimentos são precisamente conhecidos, denominados "baselines". Treze ciclistas, utilizando bicicletas com um contador que mede o número de voltas da roda diateira, percorrem todo o percurso, anotando as medições para cada trecho. O número de trechos cujo verdadeiro comprimento é conhecido são 12, restando 13 trechos cujos comprimentos devem ser estimados. Considere as seguintes suposições:

$$
y_{i j}=\beta_{j} z_{i}+e_{i j 1}, \quad i=1,2, \ldots, 12 ; j=1,2, \ldots, 13,
$$

$\operatorname{com} e_{i j 1} \sim N\left(0, s_{i j}^{-1} \sigma^{2}\right)$ e

$$
y_{k j 2}=\beta_{j} x_{k}+e_{k j 2}, \quad k=1,2, . ., 13
$$

com $e_{k j 2} \sim N\left(0, s_{k j}^{-1} \sigma^{2}\right)$, onde $y_{i j 1}$ and $y_{k j 2}$ são as medidas obtidas pelo j-ésimo ciclista, associada ao i-ésimo intervalo de calibração( "baseline") ou ao k-ésimo intervalo desconhecido, respectivamente. Os verdadeiros comprimentos conhecidos são denotados por $z_{i}$ e os desconhecidos por $x_{k}$. O interesse é estimar o comprimento total desconhecido, $T=\sum_{k=1}^{13} x_{k}$.

A principal diferença entre esta situação e o desenvolvimento feito durante todo o capítulo é que temos um vetor $\boldsymbol{\beta}=\left(\beta_{1}, \beta_{2}, \ldots, \beta_{13}\right)$, onde $\beta_{j}$ esta associado ao j-ésimo ciclista e um vetor $\boldsymbol{x}=\left(x_{1}, x_{2}, . ., x_{13}\right)$, que devem ser estimados. A extensão dos resultados obtidos na proposição 3.3 , é imediata para esta situação se considerarmos distribuições a priori independentes para os $\beta_{j}^{\prime} s$ e os $x_{k}^{\prime} s$.

Considere

$$
\bar{y}_{j 2}=\beta_{j} \bar{x}+e_{j 2}^{*},
$$


onde $e_{j 2}^{*} \sim N\left(0, \bar{s}_{j}^{-1} \sigma^{2}\right)$, e ainda, que $s_{i j}$ e $s_{k j}$ não dependem do cilista, então $s_{i j}^{-1}=\lambda_{i}, \quad i=$ $1,2, . ., 13$ e $\bar{s}_{j}=\lambda_{0}$ e $\lambda_{*}=\left(\lambda_{0}, \lambda_{1}, \ldots, \lambda_{12}\right)$.

A análise do modelo foi implementada no BUGS, ver Spiegelhalter, Thomas, Best and Gilks (1994), considerando-se as seguintes distribuições a priori:

(i) cada elemento do vetor $\lambda_{*}$ tem distribuição $I G\left(\frac{\nu}{2}, \frac{\nu}{2}\right)$, independente dos demais;

(ii) $\beta_{j} \sim N\left(0,10^{4}\right), j=1,2, . ., 13$ independentes $\mathrm{e}$

(iii) $\bar{x} \sim N\left(1000,10^{6}\right)$.

O procedimento utilizado pelo BUGS para gerar uma amostra de tamanho $m$ da distribuição a posteriori conjunta, considera uma única cadeia de Markov. A partir de um número $t$ de iterações considera-se que a cadeia convergiu e então os valores obtidos a partir daí formam uma amostra da distribuição de interesse. A análise dessa convergência foi verificada a partir do procedimento proposto por Geweke (ver apêndice), baseado em técnicas de séries temporais.

Os resultados obtidos são apresentados nas tabelas 3.1 e 3.2. Na tabela 3.1, além das estimativas para a média e desvio padrão a posteriori de $\bar{x}$, apresentamos os valores de $t$, número de iterações iniciais desconsideradas e da estatística $z$, que tem distribuição aproximadamente normal e testa a hipótese de convergência da cadeia.

Tabela 3.1: Média e desvio padrão a posteriori de $\bar{x}, t$ e $z$

\begin{tabular}{cccccccc}
\hline & & & & & & & \\
$\nu$ & 3 & 4 & 5 & 10 & 30 & 60 & 100 \\
\hline & & & & & & & \\
$\hat{x}$ & 2376.4 & 2376.5 & 2376.5 & 2376.8 & 2377.6 & 2377.7 & 2377.8 \\
$\mathrm{sd}$ & 0.6308 & 0.6215 & 0.5715 & 0.5369 & 0.4061 & 0.3646 & 0.3415 \\
$\mathrm{t}$ & 1200 & 1200 & 1200 & 900 & 700 & 700 & 600 \\
$\mathrm{z}$ & -0.69 & -0.90 & -0.91 & -0.91 & -0.08 & -0.42 & -0.57 \\
\hline
\end{tabular}

A figura 3.1, representa uma saída típica do programa BUGS e apresenta as densidades a posteriori dos parâmetros do modelo, onde beta.bar denota a média dos $\beta_{1}, \ldots, \beta_{13} ; \tau$ denota $1 / \sigma^{2}, \mathrm{wN}=\lambda_{0}$ e $\mathrm{xN}=\bar{x}$. Além disso, nós fixamos $\nu=100, t=600$ (o tamanho da cadeia) e $m=1000$ (o tamanho da amostra). 
Figura 3.1: Densidade a Posterior (Gibbs)

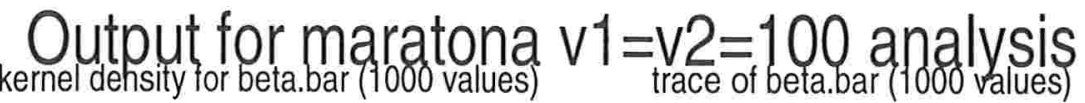
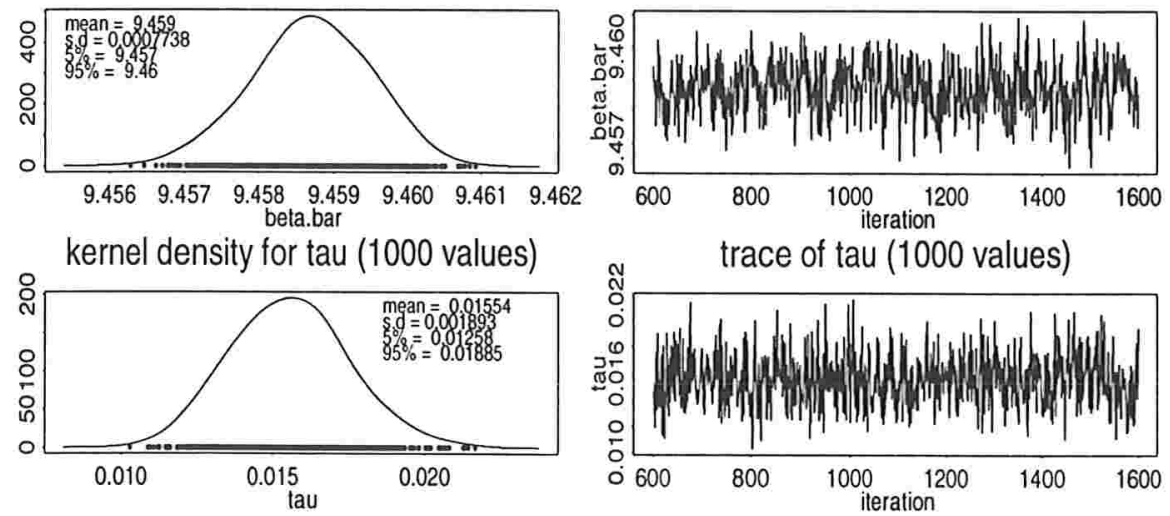

kernel density for wN (1000 values) trace of WN (1000 values)
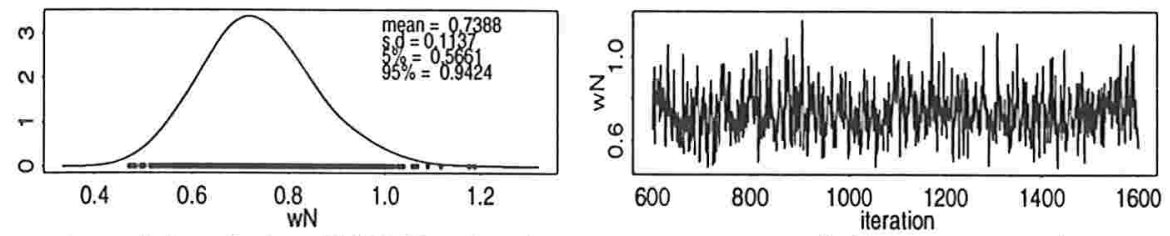

kernel density for $\mathrm{xN}$ (1000 values)

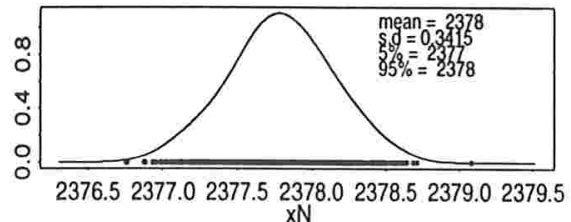

trace of $\mathrm{xN}$ (1000 values)

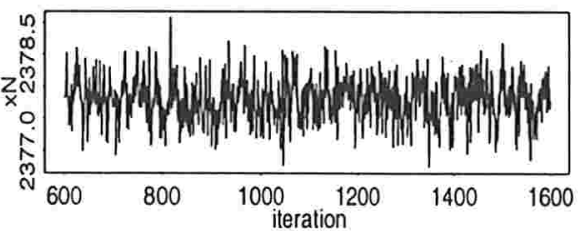

A Tabela 2 mostra valores estimados para a média e desvio padrão a posteriori de $T$ (o comprimento total desconhecido do percurso), para vários valores de $\nu$, $\operatorname{com} \nu=\nu_{1}=\nu_{2}$.

Tabela 3.2: Média e desvio padrão a posteriori de $T_{N}$

\begin{tabular}{cccccccc}
$\nu$ & 3 & 4 & 5 & 10 & 30 & 60 & 100 \\
\hline & & & & & & & \\
$\hat{T}$ & 30894 & 30894 & 30895 & 30898 & 30909 & 30911 & 30911 \\
$\mathrm{sd}$ & 8.20 & 8.08 & 7.43 & 6.98 & 5.28 & 4.74 & 4.44 \\
\hline
\end{tabular}

Os resultados da tabela indicam que a estimativa pontual do valor desconhecido varia de $30894 \mathrm{~m}$ a $30911 \mathrm{~m}$, com desvio padrão entre $8.20 \mathrm{~m}(\nu=3)$ e $4.44 \mathrm{~m}(\nu=100)$. 
Os valores obtidos por Smith and Corbett (1987) considerando o modelo normal homocedástico e aproximação normal para distribuição a posteriori são $30904 \mathrm{~m}$ (média) e 2 m (desvio padrão). O desvio padrão encontrado por Smith and Corbett é menor do que o obtido considerando-se o modelo $t$-Student. Entretanto, isso depende das suposições de normalidade assumidas.

Ainda que nosso modelo, considerando erros $t$-Student, seja mais geral e inclua o modelo normal como caso particular, ele requer a especificação dos graus de liberdades, $\nu$. Uma análise mais completa deste problema deveria incorporar no modelo uma distribuição a priori para o parâmetro $\nu$. Entretanto, um tratamento mais simples e imediato pode ser dado explorando-se a análise dos gráficos de quantis, $Q-Q$-plots. Vários gráficos representando os $Q-Q$-plots dos valores dos resíduos, sob o modelo considerado, e a distribuição teórica, são apresentados na figura 3.2. Após a análise dos gráficos concluímos que o modelo com maior valor para $\nu$, é o mais adequado. Então, a hipótese de normalidade parece ser justificada para o problema em questão.

Figura 3.2: $Q-Q$-plots ( $t$-modelo $\mathrm{x}$ resíduos)
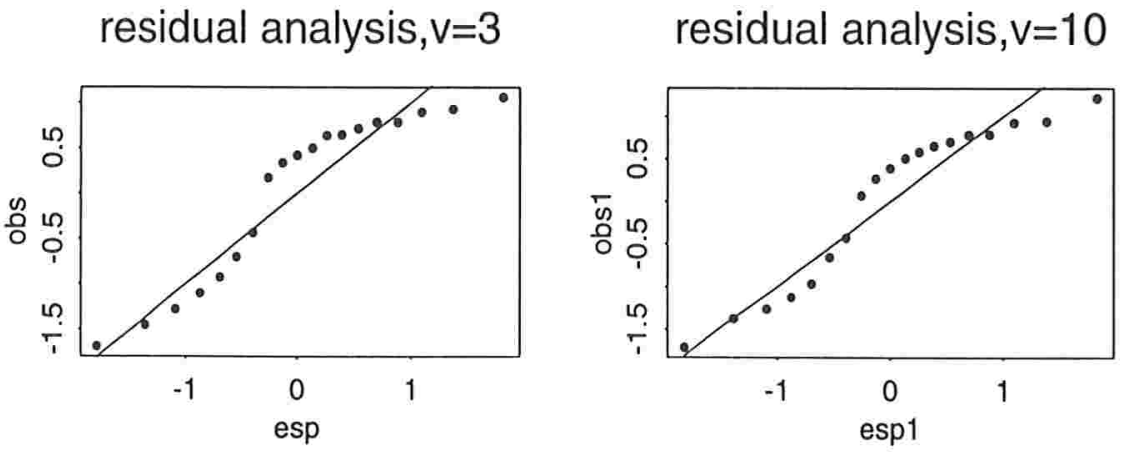

residual analysis, $\mathrm{v}=30$

residual analisis, $\mathrm{v}=100$
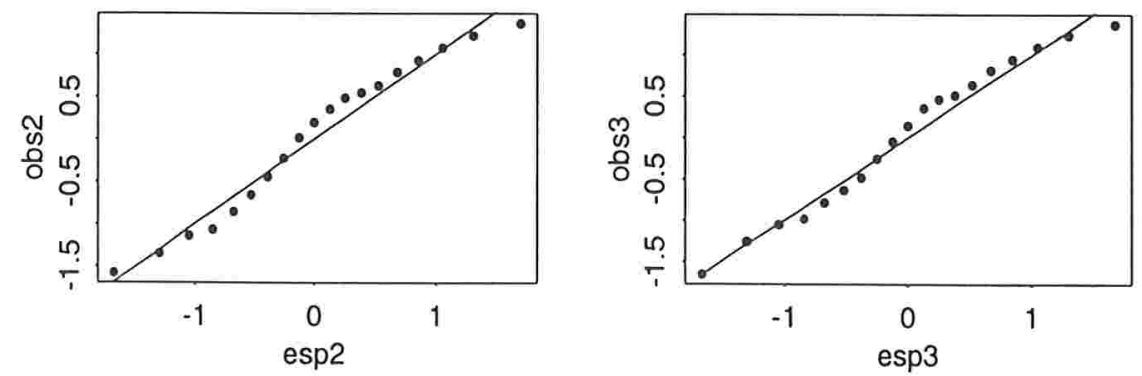


\section{Capítulo 4}

\section{Extensões multivariadas para o modelo de calibração elíptico}

\subsection{Introdução}

Neste capítulo consideramos que tanto a resposta $\boldsymbol{y}_{0}$, quanto a quantidade de interesse $\boldsymbol{x}_{0}$, são vetores. Nosso objetivo é encontrar uma densidade a posteriori multivariada para um vetor desconhecido $\boldsymbol{x}_{0}$ após a observação de um vetor de respostas $\boldsymbol{y}_{0}$ e do experimento de calibração.

Mantendo-se a suposição de linearidade temos que

$$
\boldsymbol{y}_{i}=\boldsymbol{B}^{t} \boldsymbol{x}_{i}+e_{i}, \quad i=0,1, . ., n
$$

onde $\boldsymbol{y}_{i}$ e $\boldsymbol{e}_{i}$ são vetores $q \times 1, \boldsymbol{x}_{i}$ é um vetor $p$ x1 e $\boldsymbol{B}$ é uma matriz $p \times q$. Reescrevendo o modelo em forma matricial, temos

$$
Y_{*}=X_{*} B+E_{*},
$$

onde $\boldsymbol{Y}_{*}=\left(\boldsymbol{y}_{0}^{t}, \boldsymbol{y}_{1}^{t}, \ldots, \boldsymbol{y}_{n}^{t}\right)^{t}$ e $\boldsymbol{E}_{*}=\left(e_{0}^{t}, e_{1}^{t}, \ldots, e_{n}^{t}\right)^{t}$ são matrizes $(n+1) \times q$ e $\boldsymbol{X}_{*}=\left(\boldsymbol{x}_{0}^{t}, \boldsymbol{x}_{1}^{t}, \ldots, \boldsymbol{x}_{n}^{t}\right)^{t}$ é uma matriz $(n+1) \times p$.

Usualmente no modelo de regressão linear multivariado considera-se a independência entre os erros, isto é, se supõe que os $e_{i}^{\prime} s$ são i.i.d. com distribuição normal $q$-variada com vetor de médias zero e matriz de covariância comum, $\Sigma$. Mas, se considerarmos uma suposição análoga à do capítulo 3 , onde a independência é substituída por correlaçâo nula, teremos que pensar numa distribuição conjunta para a matriz dos erros $\boldsymbol{E}$. Nossa idéia é 
trabalhar com uma matriz elíptica. Para tal o conceito de matriz elíptica será apresentado na próxima seção.

Nas seções 4.3 e 4.4 desenvolvemos resultados análogos aos obtidos no capítulo anterior. Assim, por exemplo, no caso do modelo dependente mostramos que o resultado obtido por Brown (1982) sob normalidade é mantido quando os erros são elípticos, desde que seja considerada uma distribuição a priori adequada (imprópria) para a matriz de dispersão.

\subsection{As matrizes elípticas}

Nesta seção introduzimos as distribuições elípticas de uma maneira diferente da apresentada no capítulo 3. A condição para que um vetor aleatório seja elíptico apresentada a seguir é mais geral do que a apresentada anteriormente. Além disso, é mais adequada à extensão do conceito de elipticidade para uma matriz.

Em primeiro lugar é conviniente definirmos um vetor aleatório esférico, que é um caso particular do vetor elíptico. Para isso, introduzimos a seguinte notação:

$\boldsymbol{Y} \stackrel{d}{=} \boldsymbol{X}$ significa que as quantidades aleatórias $\boldsymbol{Y}$ e $\boldsymbol{X}$ tem a mesma distribuição de probabilidades;

$\mathcal{O}_{n}=\left\{\boldsymbol{\Gamma}_{n \mathrm{X} n} \mid \Gamma \boldsymbol{\Gamma}^{t}=\boldsymbol{I}\right\}$ é o conjunto das matrizes de dimensão $n \times \mathrm{x} n$ que são ortogonais;

$\psi_{\boldsymbol{x}}(\boldsymbol{s})=E\left[e^{i \boldsymbol{S}^{t} \boldsymbol{x}}\right], \boldsymbol{s} \in \mathbb{R}^{n}, i=\sqrt{-1}$, é a função característica associada ao vetor aleatório $x$;

$$
\operatorname{vec}(\boldsymbol{X})=\left[\begin{array}{c}
\boldsymbol{x}_{1} \\
\boldsymbol{x}_{2} \\
: \\
\boldsymbol{x}_{n}
\end{array}\right] \text { é um vetor aleatório }(n q) \times 1, \text { onde } \boldsymbol{X}=\left(\boldsymbol{x}_{1}, \boldsymbol{x}_{2}, \ldots, \boldsymbol{x}_{n}\right) \text { é uma matriz }
$$

$q \times n$ e cada $\boldsymbol{x}_{i}$ é um vetor $q \times 1$;

$\boldsymbol{D}>0$ significa que a matriz é definida positiva e $\boldsymbol{C} \odot \boldsymbol{D}$ é o produto de Kronecker entre matrizes (ver, por exemplo, Fang and Zhang (1990)). 
Definição 4.1 Seja $z$ um vetor aleatório de dimensão $n \times 1$. $z$ tem distribuição esférica, se

$$
z \stackrel{d}{=} \Gamma z, \quad \forall \Gamma \in \mathcal{O}_{n}
$$

Em palavras, as distribuições esféricas são aquelas com a propriedade de invariância sob transformações ortogonais.

Uma consequência importante da definição 4.1. é que, se $\psi_{\boldsymbol{z}}$ é a função característica associada a um vetor esférico $z$ então, existe uma função caracteristica $\varphi$, associada a um escalar, tal que $\psi_{Z}(s)=\varphi\left(s^{t} s\right), \quad s \in \mathbb{R}^{n}$. A função $\varphi$ será denominada gerador característico e utilizamos a notação $z \sim S_{n}(\varphi)$ para dizer que um vetor $\boldsymbol{z}$ tem distribuição esférica.

Definição 4.2 Sejam $\boldsymbol{z}$ e $\boldsymbol{y}$ vetores aleatórios de dimensões $k \times 1$ e $n \times 1$, respectivamente, $\boldsymbol{y}$ tem distribuição elíptica com parâmetros $\boldsymbol{\mu}_{n \times 1}$ e $\boldsymbol{\Sigma}_{n \times n}$, se

$$
y=\mu+A z
$$

onde $\boldsymbol{z} \sim S_{k}(\varphi)$ e $\boldsymbol{A} \boldsymbol{A}^{t}=\boldsymbol{\Sigma}$ com Posto $(\boldsymbol{\Sigma})=k$.

Os parâmetros $\boldsymbol{\mu}$ e $\boldsymbol{\Sigma}$ representam, respectivamente, os parâmetros de locação e de dispersão e utilizamos a notação $\boldsymbol{y} \sim E l_{n}(\boldsymbol{\mu}, \boldsymbol{\Sigma} ; \varphi)$. A diferença desta notação, em relação a que vinha sendo utilizada até agora, está em substituir a função geradora $g$ pelo gerador característico $\varphi$. Isto deve-se ao fato de que, mesmo que não exista a função densidade de probabilidade de um vetor aleatório, sua função característica sempre existe e é possível definir elipticidade através desta. Observe que um vetor esférico é um caso particular de uma distribuição elíptica $\operatorname{com} \boldsymbol{\mu}=0$ e $\boldsymbol{\Sigma}$ é diagonal.

Não é difícil verificar que a definição 4.2 implica na definição de distribuição elíptica que vinha sendo utilizada até agora. De fato, no caso de existir a função densidade de um vetor aleatório esférico, esta deve ser da forma $g\left(s^{t} s\right), s \in \mathbb{R}^{n}$. Se considerarmos a relação $\boldsymbol{y}=\boldsymbol{\mu}+\boldsymbol{A} \boldsymbol{z}, \operatorname{com} k=n$, então existe $\boldsymbol{\Sigma}^{-1} \mathrm{e}$

$$
z=\Sigma^{-\frac{1}{2}}(y-\mu)
$$

O jacobiano da transformação é $|\Sigma|^{-\frac{1}{2}}$ e portanto,

$$
f(\boldsymbol{y} \mid \boldsymbol{\mu}, \boldsymbol{\Sigma})=|\Sigma|^{-\frac{1}{2}} g\left((\boldsymbol{y}-\boldsymbol{\mu})^{t} \Sigma^{-1}(\boldsymbol{y}-\boldsymbol{\mu})\right)
$$


Vamos considerar agora $Z$ uma matriz aleatória de dimensão $n \times q$, de modo que $Z=\left(z_{(1)}, z_{(2)}, \ldots, z_{(q)}\right)$ e $z_{(i)}$ é um vetor $n \times 1$, para $i=1,2, . ., n$. A definição de matriz esférica, aqui apresentada, será similar a definição 1.1.. Esse tipo de construção foi dada por Dawid (1977) e a distribuição é denominada esférica pela esquerda. Existe mais de uma maneira de definir esfericidade para uma matriz, e estas distintas definições levam a diferentes classes de matrizes elípticas, ver Fang and Zhang (1990). Neste trabalho vamos considerar apenas esfericidade pela esquerda, desse modo quando nos referirmos a uma matriz esférica estará implicito que definição de esfericidade estamos utilizando.

Definição 4.3 Seja $Z$ uma matriz aleatória $n \times q$, então $Z$ tem distribuição esférica se

$$
Z \stackrel{d}{=} \Gamma Z, \quad \forall \Gamma \in \mathcal{O}_{n}
$$

Se $Z$ é uma matriz esférica, sua função característica é da forma $\varphi\left(S^{t} S\right)$, onde $S$ é uma matriz $n \times q$. Utilizamos a notação $Z \sim S_{n x q}(\varphi)$. No caso de existir a função densidade para $Z$ ela tem a forma $\boldsymbol{g}\left(\boldsymbol{S}^{t} \boldsymbol{S}\right)$, onde $g$ é uma função da classe das matrizes $q \times q$ em $\mathbb{R}$.

Definição 4.4 Sejam $\boldsymbol{Z}$ e $\boldsymbol{Y}$ matrizes aleatórias de dimensões $n \times p$ e $n \times q$, respectivamente, então $\boldsymbol{Y}$ tem distribuição elíptica, se

$$
\boldsymbol{Y}=\boldsymbol{M}+Z \boldsymbol{A}, \quad \boldsymbol{A} \boldsymbol{A}^{t}=\Sigma, Z \sim S_{n \times p}(\varphi)
$$

para $\boldsymbol{M}$ e $\boldsymbol{A}$ matrizes $n \times q$ e $p \times q$, respectivamente.

Da última definição derivamos que a função densidade de probabilidades para a matriz $\boldsymbol{Y}$, quando existir, é da forma

$$
f(\boldsymbol{Y} \mid M, \boldsymbol{\Sigma})=|\Sigma|^{-\frac{n}{2}} \boldsymbol{g}\left(\boldsymbol{\Sigma}^{-\frac{1}{2}}(\boldsymbol{Y}-M)^{t}(\boldsymbol{Y}-\boldsymbol{M}) \boldsymbol{\Sigma}^{-\frac{1}{2}}\right) .
$$

Nesse caso, denotamos $\boldsymbol{Y} \sim E l_{n x q}(\boldsymbol{\mu}, \boldsymbol{\Sigma} ; \boldsymbol{g})$.

Uma propriedade útil que os vetores elípticos possuem e é mantida para as matrizes elípticas é a de que sob uma transformação linear a elipticidade se mantem. Isto é, se $\boldsymbol{Y} \sim E l_{n x q}(M, \Sigma ; \varphi)$ entào, $M_{1}+D_{1} \boldsymbol{Y} \sim E l_{m x q}\left(M_{1}+D_{1} M, D_{1} \Sigma D_{1}^{t} ; \varphi\right)$, onde $M_{1}$ e $D_{1}$ são matrizes $m \times q$ e $m \times n$, respectivamente. 
Exemplo 4.1 A matriz-variada normal. Seja $\boldsymbol{X}$ uma matriz $n \times q$, tal que

$$
\operatorname{Vec}\left(\boldsymbol{X}^{t}\right) \sim N_{n q}(\boldsymbol{\mu}, C \otimes \boldsymbol{D})
$$

onde $\boldsymbol{\mu}=\operatorname{Vec}(\boldsymbol{M}), \boldsymbol{M}, \boldsymbol{C}, \boldsymbol{D}$, são matrizes $n \times q, n \times n$ e $q \times q$, respectivamente, $\operatorname{com} C>0$ e $\boldsymbol{D}>0$. Então, $\boldsymbol{X}$ é uma matriz-variada normal e denotamos por

$$
\boldsymbol{X} \sim N_{n x q}(M, C \otimes D) .
$$

Além disso, a f.d.p. associada a matriz $\boldsymbol{X}$ pode ser escrita como

$$
f(X \mid M, C, D)=(2 \pi)^{-\frac{n q}{2}}|C|^{-\frac{q}{2}}|D|^{-\frac{n}{2}} e^{t r\left[-\frac{1}{2} C^{-1}(X-M) D^{-1}(X-M)^{t}\right]} .
$$

No caso particular em que $C=\boldsymbol{I}_{n}$ e $\boldsymbol{D}=\Sigma$, temos que

$$
\begin{gathered}
f(\boldsymbol{X} \mid \boldsymbol{M}, \boldsymbol{\Sigma})=(2 \pi)^{-\frac{n q}{2}}|\Sigma|^{-\frac{n}{2}} e^{\operatorname{tr}\left[-\frac{1}{2}(\boldsymbol{X}-\boldsymbol{M}) \boldsymbol{\Sigma}^{-1}(\boldsymbol{X}-\boldsymbol{M})^{t}\right]}= \\
=(2 \pi)^{-\frac{n q}{2}}|\Sigma|^{-\frac{n}{2}} e^{\operatorname{tr}\left[-\frac{1}{2} \boldsymbol{\Sigma}^{-\frac{1}{2}}(\boldsymbol{X}-\boldsymbol{M})^{t}(\boldsymbol{X}-\boldsymbol{M}) \boldsymbol{\Sigma}^{-\frac{1}{2}}\right]} .
\end{gathered}
$$

Portanto, a distribuição $N_{n x q}(M, I \otimes \Sigma)$ é uma elíptica, $E l_{n x p}(\boldsymbol{M}, \boldsymbol{\Sigma} ; \boldsymbol{g})$ com

$$
g(u)=(2 \pi)^{-\frac{n q}{2}} e^{-\operatorname{tr}\left(-\frac{u}{2}\right)} .
$$

A construção que estamos utilizando para elipticidade de uma matriz implica que as linhas da matriz não são correlacionadas e a correlação entre as colunas é representada pela matriz $\Sigma$.

Algumas propriedades interessantes das matrizes normais, são apresentadas a seguir, onde $\boldsymbol{X} \sim N_{n x q}(\boldsymbol{M}, \boldsymbol{C} \otimes \boldsymbol{D}), \quad \boldsymbol{X}_{i} \sim N_{n x q}\left(\boldsymbol{M}_{i}, \boldsymbol{\Sigma}_{i}\right), \quad i=1,2, \ldots, k$, independentes, e $\boldsymbol{G}_{m x n}, \boldsymbol{H}_{q x m}, \boldsymbol{A}_{n x q}$ são matrizes de constantes. Então,

1) $G X \sim N_{m x q}\left(G M,\left(G C G^{t}\right) \otimes D\right)$,

2) $\boldsymbol{X} \boldsymbol{H} \sim N_{n x m}\left(\boldsymbol{M} \boldsymbol{H}, \boldsymbol{C} \otimes\left(\boldsymbol{H}^{t} \boldsymbol{D} \boldsymbol{H}\right)\right)$,

3) $\boldsymbol{X}+\boldsymbol{A} \sim N_{n x q}(\boldsymbol{M}+\boldsymbol{A}, \boldsymbol{C} \otimes \boldsymbol{D})$ e

4) $\sum_{i=1}^{k} \boldsymbol{X}_{i} \sim N_{n x q}\left(\sum_{i=1}^{k} M_{i}, \boldsymbol{I} \odot \sum_{i=1}^{k} \Sigma_{i}\right)$ 


\subsection{O modelo elíptico dependente multivariado}

Vamos considerar o modelo de calibração dado em 4.1 acrescentando a seguinte suposição para a matriz de erros,

$$
E \sim E l_{(n+1) x q}(\mathrm{O}, \boldsymbol{\Sigma} ; \boldsymbol{g})
$$

Portanto,

$$
\left.Y_{*} \mid X_{*}, B, \Sigma \sim E l_{(n+1) x q}\left(X_{*} B, \Sigma ; g\right)\right)
$$

De 4.2 , temos que

$$
f\left(\boldsymbol{Y}_{*} \mid \boldsymbol{X}_{*}, \boldsymbol{B}, \boldsymbol{\Sigma}\right)=|\boldsymbol{\Sigma}|^{-\frac{n+1}{2}} g\left(\boldsymbol{\Sigma}^{-\frac{1}{2}}\left(\boldsymbol{Y}_{*}-\boldsymbol{X}_{*} \boldsymbol{B}\right)^{t}\left(\boldsymbol{Y}_{*}-\boldsymbol{X}_{*} \boldsymbol{B}\right) \boldsymbol{\Sigma}^{-\frac{1}{2}}\right),
$$

Lema 4.1 A função geradora $g$, associada a uma matriz aleatória elíptica de dimensão $n \times q$, deve satisfazer a seguinte condição

$$
\int|\boldsymbol{W}|^{\frac{(n-q-1)}{2}} g(\boldsymbol{W}) d \boldsymbol{W}=\frac{\Gamma_{q}\left(\frac{n}{2}\right)}{\pi^{\frac{n q}{2}}},
$$

onde $\boldsymbol{W}$ é uma matriz $q \times q, \Gamma_{p}($.$) é a função gama q-variada, isto é, \Gamma_{q}\left(\frac{n}{2}\right)=\pi^{\frac{q(1-q)}{4}} \prod_{i=1}^{q} \Gamma\left(\frac{n-i+1}{2}\right)$.

A próxima etapa consiste em definir as distribuições a priori para as quantidades desconhecidas $\boldsymbol{x}_{0}, \boldsymbol{B}$ e $\boldsymbol{\Sigma}$. A única distribuição a priori totalmente especificada será a distribuição da matriz de dispersão $\Sigma$. Nesse caso, analogamente a análise univariada, usaremos uma distribuição imprópria. Para as demais quantidades consideramos apenas densidades genericamente denotadas por $p\left(\boldsymbol{x}_{0} \mid \boldsymbol{X}\right)$ e $p(\boldsymbol{B})$. Além disso, estamos supondo independência a priori entre $\boldsymbol{x}_{0}$ e $(\boldsymbol{B}, \boldsymbol{\Sigma})$.

Teorema 4.1 Sob 4.3, se $p(\boldsymbol{B}, \boldsymbol{\Sigma} \mid \boldsymbol{X}) \propto|\boldsymbol{\Sigma}|^{-\frac{q+1}{2}} p(\boldsymbol{B})$, então $p\left(\boldsymbol{x}_{0} \mid \boldsymbol{Y}_{*}\right)$ não depende de $g$ e é dada por

$$
p\left(\boldsymbol{x}_{0} \mid \boldsymbol{Y}_{*}, \boldsymbol{X}\right) \propto p\left(\boldsymbol{x}_{0} \mid \boldsymbol{X}\right) \int_{\boldsymbol{B}}\left|(n-q) \boldsymbol{S}_{*}^{2}+\left(\boldsymbol{B}-\hat{\boldsymbol{B}}_{*}\right)^{t} \boldsymbol{X}_{*}^{t} \boldsymbol{X}_{*}\left(\boldsymbol{B}-\hat{\boldsymbol{B}}_{*}\right)\right|^{-\frac{(n+q)}{2}} p(\boldsymbol{B}) d \boldsymbol{B},
$$

onde

$$
\hat{\boldsymbol{B}}_{*}=\left(\boldsymbol{X}_{*}^{t} \boldsymbol{X}_{*}\right)^{-1} \boldsymbol{X}_{*}^{t} \boldsymbol{Y}_{*} e \boldsymbol{S}_{*}^{2}=\frac{\left(\boldsymbol{Y}_{*}-\boldsymbol{X}_{*} \hat{\boldsymbol{B}}\right)^{t}\left(\boldsymbol{Y}_{*}-\boldsymbol{X}_{*} \hat{\boldsymbol{B}}\right)}{n-q}, n>q .
$$


Prova: Da relação (2.10), capítulo 2, temos que

$$
p\left(\boldsymbol{x}_{0} \mid \boldsymbol{Y}_{*}, \boldsymbol{X}\right) \propto p\left(\boldsymbol{x}_{0} \mid \boldsymbol{X}\right) \underset{(\boldsymbol{B}, \boldsymbol{\Sigma})}{\int} p\left(\boldsymbol{y}_{0} \mid \boldsymbol{B}, \boldsymbol{\Sigma}, \boldsymbol{x}_{0}\right) p(\boldsymbol{B}, \boldsymbol{\Sigma} \mid \boldsymbol{Y}, \boldsymbol{X}) d(\boldsymbol{B}, \boldsymbol{\Sigma}),
$$

onde $\boldsymbol{X}=\left(\boldsymbol{x}_{1}^{t}, \ldots, \boldsymbol{x}_{n}^{t}\right)^{t}$ e $\boldsymbol{Y}=\left(\boldsymbol{y}_{1}^{t}, \ldots, \boldsymbol{y}_{n}^{t}\right)^{t}$. Esta expressão pode ser reescrita como

$$
\begin{gathered}
p\left(\boldsymbol{x}_{0} \mid \boldsymbol{Y}_{*}, \boldsymbol{X}\right) \propto p\left(\boldsymbol{x}_{0} \mid \boldsymbol{X}\right) \int_{\boldsymbol{B}} \int_{\boldsymbol{\Sigma}} V_{\boldsymbol{Y}_{*}}\left(\boldsymbol{B}, \boldsymbol{\Sigma}, \boldsymbol{x}_{0}\right) p(\boldsymbol{B}, \boldsymbol{\Sigma}) d \boldsymbol{\Sigma} d \boldsymbol{B}= \\
=p\left(\boldsymbol{x}_{0} \mid \boldsymbol{X}\right) \int_{\boldsymbol{B}} p(\boldsymbol{B}) \int_{\boldsymbol{\Sigma}} V_{\boldsymbol{Y}_{*}}\left(\boldsymbol{B}, \boldsymbol{\Sigma}, \boldsymbol{x}_{0}\right) p(\boldsymbol{\Sigma}) d \boldsymbol{\Sigma} d \boldsymbol{B} .
\end{gathered}
$$

De 4.5 segue que é suficiente considerar a integral mais interna e mostrar que ela não depende de $\boldsymbol{g}$. Assim,

$$
\int_{\Sigma} V_{Y_{*}}\left(\boldsymbol{B}, \boldsymbol{\Sigma}, \boldsymbol{x}_{0}\right)|\boldsymbol{\Sigma}|^{-\frac{(q+1)}{2}} d \boldsymbol{\Sigma}=\int_{\boldsymbol{\Sigma}}|\boldsymbol{\Sigma}|^{-\frac{(n+q+2)}{2}} g\left[\boldsymbol{\Sigma}^{-\frac{1}{2}}\left(\boldsymbol{Y}_{*}-\boldsymbol{X}_{*} \boldsymbol{B}\right)^{t}\left(\boldsymbol{Y}_{*}-\boldsymbol{X}_{*} \boldsymbol{B}\right) \boldsymbol{\Sigma}^{-\frac{1}{2}}\right] d \boldsymbol{\Sigma}
$$

Considerando a seguinte transformação

$$
W=\Sigma^{-\frac{1}{2}}\left(Y_{*}-X_{*} B\right)^{t}\left(Y_{*}-X_{*} B\right) \Sigma^{-\frac{1}{2}}
$$

e utilizando-se as propriedades de derivação para matrizes, ver Fang and Zhang (1990), temos

$$
d \boldsymbol{W}=|\boldsymbol{\Sigma}|^{-(q+1)}\left|\left(\boldsymbol{Y}_{*}-\boldsymbol{X}_{*} \boldsymbol{B}\right)^{t}\left(\boldsymbol{Y}_{*}-\boldsymbol{X}_{*} \boldsymbol{B}\right)\right|^{q} d \boldsymbol{\Sigma} .
$$

Substituindo-se 4.7 em 4.6, temos que

$$
\int_{\Sigma} V_{Y_{*}}\left(\boldsymbol{B}, \Sigma, \boldsymbol{x}_{0}\right)|\Sigma|^{-\frac{(q+1)}{2}} d \Sigma=\int_{W}|\Sigma|^{-\frac{(n-q)}{2}}\left|\left(\boldsymbol{Y}_{*}-\boldsymbol{X}_{*} \boldsymbol{B}\right)^{t}\left(\boldsymbol{Y}_{*}-\boldsymbol{X}_{*} \boldsymbol{B}\right)\right|^{-q} \boldsymbol{g}(\boldsymbol{W}) d \boldsymbol{W} .
$$

Além disso,

$$
|\boldsymbol{W}|=\left|\Sigma^{-\frac{1}{2}}\right|\left|\left(\boldsymbol{Y}_{*}-\boldsymbol{X}_{*} \boldsymbol{B}\right)^{t}\left(\boldsymbol{Y}_{*}-\boldsymbol{X}_{*} \boldsymbol{B}\right)\right|\left|\Sigma^{-\frac{1}{2}}\right|=\left|\Sigma^{-1}\right|\left|\left(\boldsymbol{Y}_{*}-\boldsymbol{X}_{*} \boldsymbol{B}\right)^{t}\left(\boldsymbol{Y}_{*}-\boldsymbol{X}_{*} \boldsymbol{B}\right)\right| .
$$

Então, 


$$
|\Sigma|^{-1}=|\boldsymbol{W}|\left|\left(\boldsymbol{Y}_{*}-\boldsymbol{X}_{*} \boldsymbol{B}\right)^{t}\left(\boldsymbol{Y}_{*}-\boldsymbol{X}_{*} \boldsymbol{B}\right)\right|^{-1} .
$$

Utilizando 4.9 , temos que 4.8 é igual a

$$
\begin{aligned}
& \int_{W}|\boldsymbol{W}|^{\frac{(n-q)}{2}}\left|\left(\boldsymbol{Y}_{*}-\boldsymbol{X}_{*} \boldsymbol{B}\right)^{t}\left(\boldsymbol{Y}_{*}-\boldsymbol{X}_{*} \boldsymbol{B}\right)\right|^{-\frac{(n+q)}{2}} \boldsymbol{g}(\boldsymbol{W}) d \boldsymbol{W}= \\
& =\left|\left(\boldsymbol{Y}_{*}-\boldsymbol{X}_{*} \boldsymbol{B}\right)^{t}\left(\boldsymbol{Y}_{*}-\boldsymbol{X}_{*} \boldsymbol{B}\right)\right|^{-\frac{(n+q)}{2}} \int_{\boldsymbol{W}}|\boldsymbol{W}|^{\frac{(n-q)}{2}} \boldsymbol{g}(\boldsymbol{W}) d \boldsymbol{W} .
\end{aligned}
$$

Finalmente, utilizando-se o Lema 4.1, obtemos

$$
\int_{\Sigma} V_{Y_{*}}\left(\boldsymbol{B}, \boldsymbol{\Sigma}, \boldsymbol{x}_{0}\right)|\Sigma|^{-\frac{(q+1)}{2}} d \Sigma=\left|\left(\boldsymbol{Y}_{*}-\boldsymbol{X}_{*} \boldsymbol{B}\right)^{t}\left(\boldsymbol{Y}_{*}-\boldsymbol{X}_{*} \boldsymbol{B}\right)\right|^{-\frac{(n+q)}{2}}\left[\frac{\Gamma_{q}\left(\frac{n+2}{2}\right)}{\pi \frac{(n+1)-q}{2}}\right],
$$

que não depende da função geradora $\boldsymbol{g}$. Consequentemente, $p\left(\boldsymbol{x}_{0} \mid \boldsymbol{Y}_{*}, \boldsymbol{X}\right)$ não depende de $\boldsymbol{g}$ e pode ser escrita como

$$
p\left(\boldsymbol{x}_{0} \mid \boldsymbol{Y}_{*}, \boldsymbol{X}\right) \propto p\left(\boldsymbol{x}_{0} \mid \boldsymbol{X}\right) \int_{\boldsymbol{B}}\left|\left(\boldsymbol{Y}_{*}-\boldsymbol{X}_{*} \boldsymbol{B}\right)^{t}\left(\boldsymbol{Y}_{*}-\boldsymbol{X}_{*} \boldsymbol{B}\right)\right|^{-\frac{(n+q)}{2}} p(\boldsymbol{B}) d \boldsymbol{B} .
$$

Utilizando-se a seguinte decomposição

$$
\left(\boldsymbol{Y}_{*}-\boldsymbol{X}_{*} \boldsymbol{B}\right)^{t}\left(\boldsymbol{Y}_{*}-\boldsymbol{X}_{*} \boldsymbol{B}\right)=\left(\boldsymbol{Y}_{*}-\boldsymbol{X}_{*} \hat{\boldsymbol{B}}\right)^{t}\left(\boldsymbol{Y}_{*}-\boldsymbol{X}_{*} \hat{\boldsymbol{B}}\right)+\left(\boldsymbol{B}-\hat{\boldsymbol{B}}_{*}\right)^{t} \boldsymbol{X}_{*}^{t} \boldsymbol{X}_{*}\left(\boldsymbol{B}-\hat{\boldsymbol{B}}_{*}\right)
$$

obtemos o resultado desejado.

\subsection{O modelo elíptico independente multivariado}

Antes de tratarmos do nosso problema de interesse vamos apresentar, brevemente, alguns resultados adicionais sobre distribuições de matrizes aleatórias. Para tal, é necessário lembrar as definições das distribuições Wishart e da matriz T (Press 1984).

Definição 4.5 Uma matriz $\boldsymbol{U}$ de dimensão $q \times q$ tem distribuição Wishart se sua f.d.p. é dada por

$$
p(\boldsymbol{U} \mid Q, d)=\frac{2^{\frac{-d q}{2}}}{\Gamma_{q}\left(\frac{d}{2}\right)} \frac{|Q|^{\frac{(d-q-1)}{2}}}{|\boldsymbol{U}|^{\frac{d}{2}}} e^{-\frac{1}{2} t r\left(\boldsymbol{U} Q^{-\frac{1}{2}}\right)}, \quad \boldsymbol{Q}>0, \boldsymbol{U}>0, d>q .
$$

Utilizamos a seguinte notação $U \sim W_{q}(Q, d)$. 
Lema 4.2 Seja $\boldsymbol{X}$ uma matriz $q \times d$ e $\boldsymbol{U}=\boldsymbol{X} \boldsymbol{X}^{t}$. Se $\boldsymbol{X} \sim N_{q x d}\left(0, \boldsymbol{Q} \otimes \boldsymbol{I}_{d}\right)$, então

$$
\boldsymbol{U} \sim W_{q}(Q, d) \text { e } U>0
$$

Definição 4.6 Uma matriz $\boldsymbol{T}$ de dimensão $q \times p$ tem distribuição T-matriz-variada se sua f.d.p. é dada por

$$
p(\boldsymbol{T} \mid Q, \boldsymbol{P}, \nu)=\frac{\Gamma_{q}\left(\frac{\nu}{2}\right) \pi^{-\frac{p q}{2}}|\boldsymbol{Q}|^{\frac{p}{2}}|\boldsymbol{P}|^{\frac{\nu-q}{2}}}{\Gamma_{q}\left(\frac{\nu-q}{2}\right)\left|\boldsymbol{P}+(\boldsymbol{T}-\boldsymbol{M})^{t} \boldsymbol{Q}(\boldsymbol{T}-\boldsymbol{M})\right|^{\frac{\nu}{2}}}
$$

onde $\boldsymbol{Q}, \boldsymbol{P}$ e $\boldsymbol{M}$ são matrizes de dimensões $q \times q, p \times p e q \times p$, respectivamente, $Q>0$, $\boldsymbol{P}>0$ e $\nu>p+q-1$. Utilizamos a notação $\boldsymbol{T} \sim T_{q x p}(\boldsymbol{M}, \boldsymbol{Q}, \boldsymbol{P} ; \nu)$.

Lema 4.3 (Dickey (1967)) Sejam $\boldsymbol{X}, \boldsymbol{U}$ matrizes $q \times p$ e $q \times q$, respectivamente, com $\boldsymbol{U}>0$ e $\boldsymbol{T}=\boldsymbol{X}^{t} \boldsymbol{U}^{-\frac{1}{2}}$. Se $\boldsymbol{X} \sim N_{q x p}\left(\boldsymbol{M}, \boldsymbol{I}_{q} \otimes \boldsymbol{P}\right), \boldsymbol{U} \sim W_{q}(\boldsymbol{Q}, \nu-p)$ e $\boldsymbol{U}$ é independente de $\boldsymbol{X}$, então

$$
\boldsymbol{T} \sim T_{q x p}(M, Q, P ; \nu), \quad Q>0, P>0, \nu>p+q-1
$$

Lema 4.4 Seja $\boldsymbol{\Sigma}=\boldsymbol{U}^{-1}$, onde $\boldsymbol{U} \sim W_{q}(\boldsymbol{Q}, d)$, então

$$
p(\Sigma \mid Q, d)=\frac{2^{\frac{-d q}{2}}}{\Gamma_{q}\left(\frac{d}{2}\right)}|Q|^{\frac{d}{2}}|\Sigma|^{-\frac{(d+q+1)}{2}} e^{-\frac{1}{2} \operatorname{tr}\left(\Sigma^{-1} Q\right)}, \quad \Sigma>0, Q>0, d>q .
$$

A densidade acima é denominada Wishart-invertida e denotamos por $\Sigma \sim W I_{q}(\boldsymbol{Q}, d)$.

Definição 4.7 Sejam $\boldsymbol{X}$ e $\boldsymbol{\Sigma}$ matrizes $n \times q$ e $q \times q$, respectivamente, se $\Sigma \sim W I_{q}(\boldsymbol{V}, d)$ e $\boldsymbol{X} \mid \boldsymbol{\Sigma} \sim N_{n x q}(\boldsymbol{A}, \boldsymbol{D} \otimes \boldsymbol{\Sigma})$, diremos que a distribuição conjunta de $(\boldsymbol{X}, \boldsymbol{\Sigma})$ é uma normal-Wishart-invertida e sua f.d.p. é dada por

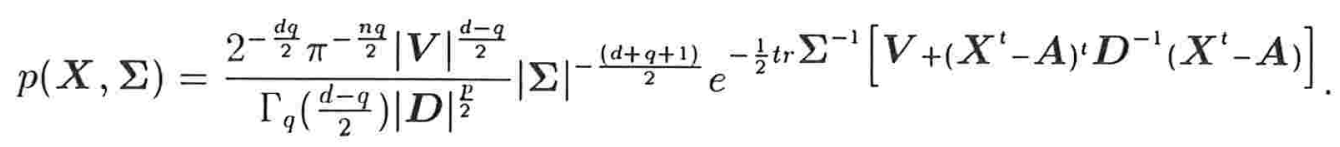

Utilizamos a notação $(\boldsymbol{X}, \boldsymbol{\Sigma}) \sim N W I(\boldsymbol{V}, d, \boldsymbol{A}, \boldsymbol{D})$.

Lema 4.5 Se $(\boldsymbol{X}, \boldsymbol{\Sigma}) \sim N W I(\boldsymbol{V}, d, \boldsymbol{A}, \boldsymbol{D})$, então

$$
\boldsymbol{X} \sim T_{n x q}(\boldsymbol{A}, \boldsymbol{D}, \boldsymbol{V} ; d) .
$$




\section{Prova:}

$$
\begin{gathered}
p(\boldsymbol{X})=\int_{\Sigma} p(\boldsymbol{X}, \boldsymbol{\Sigma}) d \boldsymbol{\Sigma} \propto \int_{\Sigma}|\Sigma|^{-\frac{d+q+1}{2}} e^{-\frac{1}{2} \operatorname{tr} \boldsymbol{\Sigma}^{-1}\left[\boldsymbol{V}+\left(\boldsymbol{X}^{t}-\boldsymbol{A}\right)^{t} \boldsymbol{D}^{-1}\left(\boldsymbol{X}^{t}-\boldsymbol{A}\right)\right]} d \boldsymbol{\Sigma} \propto \\
\propto\left|\boldsymbol{V}+\left(\boldsymbol{X}^{t}-\boldsymbol{A}\right)^{t} \boldsymbol{D}^{-1}\left(\boldsymbol{X}^{t}-\boldsymbol{A}\right)\right|^{-\frac{d}{2}} . \diamond
\end{gathered}
$$

Considere, agora, o modelo de calibração dado em 4.1, acrescentando a seguinte suposição

$$
e_{i} \mid \Sigma \stackrel{i i d}{\sim} E l_{q}(0, \Sigma ; g), \quad i=0,1, . ., n
$$

Portanto,

$$
\boldsymbol{y}_{i} \mid \boldsymbol{\Sigma}, \boldsymbol{B} \stackrel{i i d}{\sim} E l_{q}\left(\boldsymbol{B}^{t} \boldsymbol{x}_{i}, \boldsymbol{\Sigma} ; g\right), \quad i=0,1, . ., n
$$

resultando que, no caso de existir, a densidade é dada por

$$
p\left(\boldsymbol{Y}_{*} \mid \boldsymbol{X}_{*}, \boldsymbol{B}, \boldsymbol{\Sigma}\right) \propto \prod_{i=0}^{n}|\boldsymbol{\Sigma}|^{-\frac{1}{2}} g\left[\left(\boldsymbol{y}_{i}-\boldsymbol{B}^{t} \boldsymbol{x}_{i}\right)^{t} \boldsymbol{\Sigma}^{-1}\left(\boldsymbol{y}_{i}-\boldsymbol{B}^{t} \boldsymbol{x}_{i}\right)\right] .
$$

Vamos considerar somente o caso onde a a função geradora $g$ é representável. Neste caso, podemos escrever nosso modelo de forma hierárquica como segue:

(i) $\boldsymbol{y}_{i} \mid \boldsymbol{B}, \boldsymbol{\Sigma}, \boldsymbol{x}_{i}, \lambda_{i} \sim N_{q}\left(\boldsymbol{B}^{t} \boldsymbol{x}_{i}, \lambda_{i} \boldsymbol{\Sigma}\right)$

(ii) $\lambda_{0}, \lambda_{1}, \ldots, \lambda_{n}$ são v.a.i.i.d. com f.d.a. $G$.

Lembramos a notação utilizada $\boldsymbol{\lambda}_{*}=\left(\lambda_{0}, \lambda_{1}, \ldots, \lambda_{n}\right)$ e $\boldsymbol{\Lambda}_{*}=\operatorname{Diag}\left(\boldsymbol{\lambda}_{*}\right)$.

Como antes, podemos pensar em duas situações: a primeira considerando-se uma distribuição imprópria para $(\boldsymbol{B}, \boldsymbol{\Sigma})$ e a segunda utilizando-se distribuições a priori para $\boldsymbol{B}$ e $\boldsymbol{\Sigma}$ motivadas pela conjugação num problema de regressão linear multivariado.

Caso 1 : Distribuição a priori de referência

$$
p\left(B, \Sigma \mid X_{*}\right) \propto|\Sigma|^{-\frac{(q+1)}{2}} .
$$


Caso 2: Distribuição normal Wishart invertida: $(\boldsymbol{B}, \boldsymbol{\Sigma}) \sim N W I\left(\boldsymbol{V}_{0}, d_{0}, \boldsymbol{B}_{0}, \boldsymbol{M}_{\mathbf{0}}\right)$. Portanto,

$$
\begin{aligned}
& \boldsymbol{B} \mid \boldsymbol{\Sigma}, \boldsymbol{X}_{*} \sim N_{p x q}\left(\boldsymbol{B}_{0}, \boldsymbol{M}_{0} \otimes \boldsymbol{\Sigma}\right) e \\
& \boldsymbol{\Sigma} \mid \boldsymbol{X}_{*} \sim W I_{q}\left(\boldsymbol{V}_{0}, d_{0}\right)
\end{aligned}
$$

O primeiro caso não será desenvolvido neste trabalho e envolve discussão sobre a existência da distribuição a posteriori.

Proposição 4.1 Sob o modelo de calibração especificado em 4.1 e 4.11 e a hipótese de $g$ ser representável. Sejam $(\boldsymbol{B}, \boldsymbol{\Sigma}) \sim N W I\left(\boldsymbol{V}_{0}, d_{0}, \boldsymbol{B}_{0}, \boldsymbol{M}_{0}\right)$ e $p\left(\boldsymbol{x}_{0} \mid \boldsymbol{X}\right)$ uma f.d.p., então $p\left(\boldsymbol{x}_{0} \mid \boldsymbol{X}, \boldsymbol{Y}_{*}\right)$ é proporcional a

$$
p\left(x_{0} \mid \boldsymbol{X}\right) \int_{\lambda_{*}} \frac{\left|\boldsymbol{\Lambda}_{*}+\boldsymbol{X}_{*} \boldsymbol{M}_{0} \boldsymbol{X}_{*}^{t}\right|^{\frac{p}{2}}}{\left|\boldsymbol{V}_{0}+\left(\boldsymbol{Y}_{*}-\boldsymbol{X}_{*} \boldsymbol{B}_{0}\right)^{t}\left[\boldsymbol{\Lambda}_{*}+\boldsymbol{X}_{*} \boldsymbol{M}_{0} \boldsymbol{X}_{*}^{t}\right]\left(\boldsymbol{Y}_{*}-\boldsymbol{X}_{*} \boldsymbol{B}_{0}\right)\right|^{\frac{d_{0}}{2}}} d G\left(\boldsymbol{\lambda}_{*}\right)
$$

Prova: Considere $Z_{*}=\Lambda_{*}^{-\frac{1}{2}} Y_{*}$ e $V_{*}=\Lambda_{*}^{-\frac{1}{2}} \boldsymbol{X}_{*}$.

Neste caso,

$$
Z_{*} \mid \boldsymbol{B}, \boldsymbol{\Sigma}, \boldsymbol{X}_{*}, \boldsymbol{\lambda}_{*} \sim N_{n \times q}\left(\boldsymbol{V}_{*} \boldsymbol{B}, \boldsymbol{I}_{n} \otimes \boldsymbol{\Sigma}\right)
$$

e podemos escrever

$$
Z_{*}=V_{*} B+e_{*}
$$

onde $\boldsymbol{e}_{*} \mid \boldsymbol{\Sigma}, \boldsymbol{X}_{*}, \boldsymbol{\lambda}_{*} \sim N_{n \times q}\left(0, \boldsymbol{I}_{n} \otimes \boldsymbol{\Sigma}\right)$ e $\boldsymbol{B} \mid \boldsymbol{\Sigma}, \boldsymbol{X}_{*}, \boldsymbol{\lambda}_{*} \sim N_{p \times q}\left(\boldsymbol{B}_{0}, \boldsymbol{M}_{0} \otimes \boldsymbol{\Sigma}\right)$.

Utilizando-se as propriedades da matriz normal e a independência entre $\boldsymbol{e}_{*}$ e $\boldsymbol{B}$, resulta que

$$
Z_{*} \mid \Sigma, X_{*}, \lambda_{*} \sim N_{n \times q}^{r}\left(V_{*} B_{0},\left[I_{n}+V_{*} M_{0} V_{*}^{t}\right] \otimes \Sigma\right)
$$

Mas, por hipótese, $\Sigma \mid \boldsymbol{X}_{*}, \boldsymbol{\lambda}_{*} \sim W I\left(\boldsymbol{V}_{0}, d_{0}\right)$. Portanto,

$$
\left(Z_{*}, \Sigma\right) \mid X_{*}, \lambda_{*} \sim N W I\left(V_{0}, d_{0}, V_{*} B_{0},\left[\boldsymbol{I}_{n}+V_{*} M_{0} V_{*}^{t}\right]\right)
$$

Utilizando-se o lema 4.5 , obtemos 


$$
Z_{*} \mid \boldsymbol{X}_{*}, \boldsymbol{\lambda}_{*} \sim T_{n \times q}\left(d_{0}, V_{*} \boldsymbol{B}_{0},\left[\boldsymbol{I}_{n}+V_{*} M_{0} \boldsymbol{V}_{*}^{t}\right], \boldsymbol{V}_{0}\right)
$$

Portanto,

$$
\boldsymbol{Y}_{*} \mid \boldsymbol{X}_{*}, \boldsymbol{\lambda}_{*} \sim T_{n \times q}\left(d_{0}, \boldsymbol{X}_{*} \boldsymbol{B}_{0},\left[\boldsymbol{\Lambda}_{*}+\boldsymbol{X}_{*} \boldsymbol{M}_{0} \boldsymbol{X}_{*}^{t}\right], \boldsymbol{V}_{0}\right) . \diamond
$$

Proposição 4.2 Sob as condições da proposição 4.1, se $\boldsymbol{x}_{0} \sim N_{p}\left(\boldsymbol{m}_{0}, \Delta_{0}\right)$ entâo,

(i) $B \mid \Sigma, \boldsymbol{x}_{0}, \boldsymbol{\lambda}_{*}, \boldsymbol{Y}_{*} \sim N_{p x q}\left(\boldsymbol{B}_{1}, \boldsymbol{M}_{1} \otimes \Sigma\right)$,

(ii) $\boldsymbol{\Sigma} \mid \boldsymbol{B}, \boldsymbol{x}_{0}, \boldsymbol{\lambda}_{*}, \boldsymbol{Y}_{*} \sim W I\left(\boldsymbol{V}_{1}, d_{1}\right)$,

(iii) $\boldsymbol{x}_{0} \mid \Sigma, \boldsymbol{B}, \boldsymbol{\lambda}_{*}, \boldsymbol{Y}_{*} \sim N_{p}\left(\boldsymbol{m}_{1}, \boldsymbol{\Delta}_{1}\right)$,

onde

$$
\begin{aligned}
& \boldsymbol{B}_{1}=M_{1}\left[M_{0} \boldsymbol{B}_{0}+\left(\boldsymbol{X}_{*} \Lambda_{*}^{-1} \boldsymbol{X}_{*}^{t}\right) \hat{B}_{*}\right], \quad M_{1}^{-1}=M_{0}^{-1}+\left(\boldsymbol{X}_{*} \Lambda_{*}^{-1} \boldsymbol{X}_{*}^{t}\right), \\
& d_{1}=n+1+d_{0}, \quad V_{1}=V_{0}+S_{*}^{\lambda}+\left(B-\hat{B}_{*}\right)^{t} \boldsymbol{X}_{*}^{t} \Lambda_{*} \boldsymbol{X}_{*}\left(B-\hat{B}_{*}\right), \\
& \Delta_{1}=\left[\Delta_{0}^{-1}+\Delta^{-1}\right]^{-1}, \quad \boldsymbol{m}_{1}=\Delta_{1}\left[\Delta_{1}^{-1} \boldsymbol{m}_{0}+\Delta^{-1} \boldsymbol{m}\right],
\end{aligned}
$$

com

$$
\begin{gathered}
\Delta=\left(B^{t} \Sigma^{-1} B\right)^{-1}, \quad m=\Delta B^{t} \Sigma^{-1} y_{0} \\
\hat{B}_{*}=\left(X_{*}^{t} \Lambda_{*}^{-1} X_{*}\right)^{-1} \boldsymbol{X}_{*}^{t} \Lambda_{*}^{-1} Y_{*} \quad \text { e } S_{*}^{\lambda}=\left(\boldsymbol{Y}_{*}-\boldsymbol{X}_{*} \hat{B}_{*}\right)^{t} \Lambda_{*}^{-1}\left(\boldsymbol{Y}_{*}-\boldsymbol{X}_{*} \hat{B}_{*}\right) .
\end{gathered}
$$

\section{Prova:}

A função de verossimilhança associada ao modelo de calibração multivariado independente, sob a condição de $g$ ser representável, é dada por

$$
V_{Y_{*}}\left(\boldsymbol{B}, \boldsymbol{\Sigma}, \boldsymbol{x}_{0}, \boldsymbol{\lambda}_{*}\right) \propto \int_{\boldsymbol{\lambda}_{*}}|\Sigma|^{-\frac{(n+1)}{2}} e^{-\frac{1}{2} t r \Sigma^{-1}\left[S_{*}^{\lambda}+\left(\boldsymbol{B}_{-} \dot{B}_{*}\right)^{t} \boldsymbol{X}_{*}^{t} \boldsymbol{\Lambda}_{*}^{-1} \boldsymbol{X}_{*}\left(\boldsymbol{B}-\dot{B}_{*}\right)\right]} d G\left(\boldsymbol{\lambda}_{*}\right)
$$

Portanto,

$p\left(\boldsymbol{B} \mid \Sigma, \boldsymbol{x}_{0}, \boldsymbol{\lambda}_{*}, \boldsymbol{Y}_{*}\right) \propto e^{-\frac{1}{2} t r\left[\Sigma^{-1}\left(\boldsymbol{B}_{-} \dot{B}_{*}\right)^{t} \boldsymbol{X}_{*}^{t} \boldsymbol{\Lambda}_{*}^{-1} \boldsymbol{X}_{*}\left(\boldsymbol{B}_{-} \dot{\boldsymbol{B}}_{*}\right)+\Sigma^{-1}\left(\boldsymbol{B}_{-} \boldsymbol{B}_{0}\right)^{t} \boldsymbol{M}_{0}^{-1}\left(\boldsymbol{B}_{-} \boldsymbol{B}_{0}\right)\right]}$, 
resultando em

$$
p\left(B \mid \Sigma, \boldsymbol{x}_{0}, \boldsymbol{\lambda}_{*}, \boldsymbol{Y}_{*}\right) \propto e^{-\frac{1}{2} t r \Sigma^{-1}\left(\boldsymbol{B}-\boldsymbol{B}_{1}\right)^{t} \boldsymbol{M}_{1}^{-1}\left(\boldsymbol{B}-\boldsymbol{B}_{1}\right)} .
$$

Para obtenção do resultado (ii), temos que

$$
p\left(\Sigma \mid \boldsymbol{B}, \boldsymbol{x}_{0}, \boldsymbol{\lambda}_{*}, \boldsymbol{Y}_{*}\right) \propto|\Sigma|^{\frac{-(n+1)-d_{0}+q+1}{2}} e^{-\frac{1}{2} t r \Sigma^{-1}\left[\boldsymbol{S}_{*}^{\lambda}+\left(\boldsymbol{B}-\dot{\boldsymbol{B}}_{*}\right)^{t} \boldsymbol{X}_{*}^{t} \boldsymbol{\Lambda}_{*}^{-1} \boldsymbol{X}_{*}\left(\boldsymbol{B}-\hat{\boldsymbol{B}}_{*}\right)+\boldsymbol{V}_{0}\right]},
$$

resultando em

$$
p\left(\Sigma \mid \boldsymbol{B}, \boldsymbol{x}_{0}, \boldsymbol{\lambda}_{*}, \boldsymbol{Y}_{*}\right) \propto|\boldsymbol{\Sigma}|^{-\frac{d_{1}+q+1}{2}} e^{-\frac{1}{2} t r \Sigma^{-1} V_{1}}
$$

Finalmente, para a obtenção da distribuição condicional a posteriori de $\boldsymbol{x}_{0}$, temos que

$$
p\left(\boldsymbol{x}_{0} \mid \boldsymbol{B}, \boldsymbol{\Sigma}, \boldsymbol{\lambda}_{*}, \boldsymbol{Y}_{*}\right) \propto e^{-\frac{1}{2} \operatorname{tr}\left[\boldsymbol{\Delta}_{0}^{-1}\left(\boldsymbol{x}_{0}-\boldsymbol{m}_{0}\right)^{t}\left(\boldsymbol{x}_{0}-\boldsymbol{m}_{0}\right)+\boldsymbol{\Sigma}^{-1}\left(\boldsymbol{y}_{0}-\boldsymbol{B}^{t} \boldsymbol{x}_{0}\right)^{t}\left(\boldsymbol{y}_{0}-\boldsymbol{B}^{t} \boldsymbol{x}_{0}\right)\right]} .
$$

Mas,

$$
e^{-\frac{1}{2} t r\left[\Sigma^{-1}\left(\boldsymbol{y}_{0}-\boldsymbol{B}^{t} \boldsymbol{x}_{0}\right)^{t}\left(\boldsymbol{y}_{0}-\boldsymbol{B}^{t} \boldsymbol{x}_{0}\right)\right]} \propto e^{-\frac{1}{2} t r\left[\Delta^{-1}\left(\boldsymbol{x}_{0}-\boldsymbol{m}\right)^{t}\left(\boldsymbol{x}_{0}-\boldsymbol{m}\right)\right]}
$$

De fato,

$$
\begin{gathered}
e^{-\frac{1}{2} \operatorname{tr}\left[\boldsymbol{\Delta}^{-1}\left(\boldsymbol{x}_{0}-\boldsymbol{m}\right)^{t}\left(\boldsymbol{x}_{0}-\boldsymbol{m}\right)\right]}=e^{-\frac{1}{2} \operatorname{tr}\left[\boldsymbol{\Delta}^{-1}\left(\boldsymbol{x}_{0}-\boldsymbol{\Delta} \boldsymbol{B}^{t} \boldsymbol{\Sigma}^{-1} \boldsymbol{y}_{0}\right)^{t}\left(\boldsymbol{x}_{0}-\Delta \boldsymbol{B}^{t} \Sigma^{-1} \boldsymbol{y}_{0}\right)\right]} \propto \\
\propto e^{-\frac{1}{2}\left[\boldsymbol{x}_{0}^{t} \Delta^{-1} \boldsymbol{x}_{0}-2 \boldsymbol{x}_{0}^{t} \boldsymbol{\Delta}^{-1} \Delta \boldsymbol{B}^{t} \boldsymbol{\Sigma}^{-1} \boldsymbol{y}_{0}\right]}=e^{-\frac{1}{2}\left[\left(\boldsymbol{B} \boldsymbol{x}_{0}\right)^{t} \boldsymbol{\Sigma}^{-1} \boldsymbol{B} \boldsymbol{x}_{0}-2\left(\boldsymbol{B} \boldsymbol{x}_{0}\right)^{t} \boldsymbol{\Sigma}^{-1} \boldsymbol{y}_{0}\right]} \\
\propto e^{-\frac{1}{2} \operatorname{tr}\left[\boldsymbol{\Sigma}^{-1}\left(\boldsymbol{y}_{0}-\boldsymbol{B}^{t} \boldsymbol{x}_{0}\right)^{t}\left(\boldsymbol{y}_{0}-\boldsymbol{B}^{t} \boldsymbol{x}_{0}\right)\right]} .
\end{gathered}
$$

Portanto,

$$
p\left(\boldsymbol{x}_{0} \mid \boldsymbol{B}, \boldsymbol{\Sigma}, \boldsymbol{\lambda}_{*}, \boldsymbol{Y}_{*}\right) \propto e^{-\frac{1}{2} t r\left[\Delta_{0}^{-1}\left(\boldsymbol{x}_{0}-\boldsymbol{m}_{0}\right)^{t}\left(\boldsymbol{x}_{0}-\boldsymbol{m}_{0}\right)+\Delta^{-1}\left(\boldsymbol{x}_{0}-\boldsymbol{m}\right)^{t}\left(\boldsymbol{x}_{0}-\boldsymbol{m}\right)\right]}
$$

e utilizando as simplificações usuais da família conjugada da normal multivariada, obtemos o resultado desejado. $\diamond$ 


\section{Capítulo 5}

\section{Calibração em dados categorizados}

\subsection{Introdução}

Nos capítulos anteriores trabalhamos com o problema de calibração numa classe de distribuições mais ampla do que a distribuição normal, permitindo que uma quantidade maior de problemas em calibração sejam resolvidos. Entretanto, esses capítulos não contemplam os problemas onde a variável resposta é discreta. Em particular, quando a resposta é dicotômica, isto é, os resultados podem ser classificados em duas categorias distintas. Por conveniência diremos que o resultado é 0 se pertence a primeira categoria e 1 se pertence a segunda. Assim, os possíveis resultados pertencem ao conjunto $\{0,1\}$ e podemos definir uma variável discreta $y_{i}$ como o número de 1 's em $k_{i}$ respostas associadas a uma quantidade fixada $x_{i}, i=1,2, \ldots, n$. Um exemplo deste tipo de problema é a situação descrita. na introdução desse trabalho, onde $x_{i}$ é uma dose de radiação fixada e $y_{i}$ é o número de células com aberrações após a exposição a dose de radiação $x_{i}$. Supondo-se independência entre as células, o modelo tradicionalmente utilizado neste tipo de problema é o modelo binomial, que será abordado na seção 3.2. Utilizaremos a notação $y \sim B(k, p)$, para dizer que a v.a. $y$ tem distribuição binomial com parâmetros $k$ e $p$, onde $p$ representa a probabilidade associada à categoria 1 .

Na seção 3.3 apresentamos uma extensão do problema binomial onde os resultados podem ser classificados em $q$ categorias distintas, ampliando o conjunto das possíveis respostas para $\{1,2, \ldots, q\}, q \geq 2$. Neste caso, podemos definir o vetor $\boldsymbol{y}_{i}=\left(y_{i 1}, \ldots, y_{i q}\right)$ onde cada componente $y_{i j}$ representa o número de respostas na j-ésima categoria associada a quantidade $x_{i}$. Deste modo $\boldsymbol{y}_{i}$ pode ser modelado através de uma distribuição multinomial 
$q$-variada, que será denotada por $M_{q}(k, \boldsymbol{p})$, onde $\boldsymbol{p}=\left(p_{1}, \ldots, p_{q}\right)$ e cada $p_{i}$ é a probabilidade associada a i-ésima categoria. Para exemplificar este tipo de problema, mais uma vez recorremos à situação descrita na introdução. Para cada dose fixada, $x_{i}$, é observado o número de aberrações por célula, já que uma célula pode ter mais de uma aberração. Assim, $y_{i j}$ é o número de células com $(j-1)$ aberrações, $j=1,2, \ldots, q-1$, das $k_{i}$ células expostas a dose de radiação $x_{i}, i=1,2, \ldots, n$.

Ao considerarmos modelos discretos, como o modelo binomial e o modelo multinomial, a suposição de linearidade entre a resposta $y$ e a variável explicativa $x$, em geral, não é adequada. Portanto, neste capítulo estaremos lidando com modelos não lineares. Dois tipos de modelos são de imediato lembrados quando trabalhamos com a distribuição binomial, o modelo logit e o modelo probit.

Finney (1971) sugere o uso dos modelos probit e logit para estudar o problema de dose-resposta que será descrito a seguir. São considerados dois componentes: o estímulo (por exemplo, radiação) e o sujeito (por exemplo, células sanguíneas). A denominação dose é utilizada para especificar a intensidade de um estímulo a que é submetido o sujeito, após o qual espera-se uma resposta (por exemplo, deformação da célula). Denomina-se tolerância o valor limite de dose a partir do qual o sujeito apresenta uma resposta positiva. A tolerância é uma característica do sujeito e portanto irá variar na população. Denotamos por $T$ a variável aleatória que irá representar a tolerância individual. Se uma dose $d$ é aplicada a uma população, a resposta positiva é obtida nos indíviduos que apresentarem $T \leq d$. Assim, a proporção esperada de indivíduos com resposta positiva será

$$
p=P(T \leq d)=\int_{0}^{d} g(t) d t
$$

onde $g(t)$ é uma função densidade de probabilidades (f.d.p.) associada a $T$. Como a dose é, em geral, uma quantidade positiva podemos utilizar, por exemplo, a transformação $X=\log T$, e assim trabalhar com valores em $\mathbb{R}$. Nesta caso, escrevemos

$$
p=\int_{-\infty}^{x} f(t) d t,
$$

onde $f$ é uma f.d.p. em IR. As diferentes escolhas de $f$ caracterizam os modelos probit e logit; a primeira corresponde a f.d.p. normal e a segunda a f.d.p. logística.

Seja $y$ o número de respostas positivas numa amostra de $k$ sujeitos submetidos a um valor $x$. Então, $y$ pode ser modelado através da distribuição binomial com parâmetros 
$(k, p)$, isto é, $y \sim B(k, p)$, onde $p$ é dado por 5.1. O interesse é estabelecer a relação entre dose e resposta, isso pode ser encarado como um problema de regressão não linear nas quantidades originais $x$ e $y$. Entretanto, com a suposição de normalidade

$$
p=P(X \leq x)=\Phi\left(\frac{x-\mu}{\sigma}\right),
$$

onde $\Phi(z)=P(Z \leq z)$, com $Z \sim N(0,1)$. Então, $\Phi^{-1}(p)=\beta_{1}+\beta_{2} x, \operatorname{para} \beta_{1}=\mu / \sigma, \beta_{2}=$ $1 / \sigma, \beta_{1} \in \mathbb{R}$ e $\beta_{2} \in \mathbb{R}^{+}$. Obtendo-se uma relação linear entre $x$ e uma transformação de $p$, denominada transformação probit.

Analogamente, com a suposição de que a f.d.p é logística, obtem-se

$$
\log \left(\frac{p}{1-p}\right)=\beta_{1}+\beta_{2} x
$$

que é denominada transformação logit.

Há muitas semelhanças entre as curvas ajustadas pelas transformações logit e probit, para quase todo valor de $p$. As diferenças aparecem para valores muito grandes ou muito pequenos de $p$ (equivalentemente, para valores pequenos e grandes da dose, $x$ ). Estimativas para os parâmetros $\beta_{1}$ e $\beta_{2}$ podem ser obtidas utilizando-se o método de máxima verossimilhança. Entretanto, a obtenção destas estimativas é feita através de métodos numéricos como, por exemplo, o algoritmo de Newton-Raphson. Já que não existem formas analíticas explicitas para os estimadores de máxima verossimilhança (e.m.v) de $\beta_{1} \mathrm{e}$ $\beta_{2}$ (ver McCullang and Nelder (1983)) .

\subsection{O modelo binomial}

Considere o seguinte modelo de calibração

$$
y_{i} \mid x_{i}, \beta_{1}, \beta_{2} \stackrel{i n d}{\sim} B\left(k_{i}, F\left(\beta_{1}+\beta_{2} x_{i}\right)\right), i=0,1, \ldots, n,
$$

onde $F$ é a função de distribuição acumulada (f.d.a.) de uma distribuição elíptica padronizada, isto é, $F$ é a f.d.a. de uma $Z \sim E l_{1}(0,1 ; g)$.

No caso de $F$ ser a f.d.a. da distribuição normal padrão, temos o modelo probit; e quando $F$ é a f.d.a. de uma distribuição logística, o modelo logístico. Em ambos os casos, a f.d.a. $F$ esta na classe das distribuiçôes elípticas. 
De 5.2, temos que a função de verossimilhança é dada por

$$
V_{y_{*}}\left(\boldsymbol{\beta}, x_{0}\right)=\prod_{i=0}^{n}\left(\begin{array}{c}
k_{i} \\
y_{i}
\end{array}\right)\left[F\left(\beta_{1}+\beta_{2} x_{i}\right)\right]^{y_{i}}\left[1-F\left(\beta_{1}+\beta_{2} x_{i}\right)\right]^{k_{i}-y_{i}} .
$$

Com essa expressão para a função de verossimilhança fica extremamente difícil determinar analiticamente a distribuição a posteriori de $x_{0}$, mesmo para prioris de referência. Vemos duas alternativas para a solução deste problema. A primeira é procurar obter a distribuição a posteriori através de métodos numéricos de estimação e a segunda solução é trabalhar com uma aproximação assintótica para a distribuição a posteriori, que será discutida na seção 5.2.2. Na seção 5.2.1 procuramos implementar a solução numérica utilizando-se a proposta de Tanner and Wong (1987) e seu algoritmo estocástico conhecido como "data augmentation".

\subsubsection{Estimação da distribuição a posteriori via algoritmo es- tocástico}

Nesta seção utilizamos o algoritmo de Tanner and Wong (1987) conhecido como "data augmentation", que denotaremos por DA, com o objetivo de estimar a distribuição a posteriori de $x_{0}$. A idéia básica do algoritmo, na mesma linha do algoritmo EM, consiste em considerar um modelo ampliado (aumentado) através da introdução de variáveis artificiais (não observadas), denominadas variáveis latentes, de modo que seja mais fácil trabalhar neste modelo ampliado. Para ilustrar a idéia desse algoritmo denotamos por $\boldsymbol{y}$ o vetor aleatório original, que será observado, $\boldsymbol{w}$ o vetor aleatório associado às variáveis latentes e $\theta$ o parâmetro de interesse. Para obtermos a densidade a posteriori de interesse, $p(\theta \mid \boldsymbol{y})$, geram-se vários valores de $\boldsymbol{w}$ da densidade preditiva $p(\boldsymbol{w} \mid \boldsymbol{y})$ e calcula-se a média de $p(\theta \mid \boldsymbol{y}, \boldsymbol{w})$ sobre estes valores. Como $p(\boldsymbol{w} \mid \boldsymbol{y})$ depende de $p(\theta \mid \boldsymbol{y})$, o algoritmo é obtido de forma iterativa, isto é, os valores gerados de $\boldsymbol{w}$ na etapa seguinte irão depender da estimativa da densidade a posteriori no instante atual, e essa por sua vez é atualizada atravéz dos novos valores gerados para $\boldsymbol{w}$. As relações entre estas densidades são dadas pelas equações abaixo que são denominadas, respectivamente, de equação a posteriori e equação preditiva.

$$
p(\theta \mid \boldsymbol{y})=\int_{W} p(\theta \mid \boldsymbol{y}, \boldsymbol{w}) p(\boldsymbol{w} \mid \boldsymbol{y}) d \boldsymbol{w}
$$




$$
p(\boldsymbol{w} \mid \boldsymbol{y})=\int_{\theta} p(\boldsymbol{w} \mid \phi, \boldsymbol{y}) p(\phi \mid \boldsymbol{y}) d \phi .
$$

Como observado por Tanner (1991), as sequências de valores, $\theta^{(1)}, \theta^{(2)}, \ldots, \theta^{(i)}$ e $\boldsymbol{w}^{(1)}, \boldsymbol{w}^{(2)}, \ldots, \boldsymbol{w}^{(i)}$, geradas de forma iterativa através das equações 5.4 e 5.5 , formam um processo markoviano com função de transição $k(\theta, \phi)=\int p(\theta \mid \boldsymbol{w}, y) p(\boldsymbol{w} \mid \phi, \boldsymbol{y}) d \boldsymbol{w}$. Sob condições de regularidade, esse processo é markoviano ergódico e obtem-se a convergência em distribuição. Isto é, quando $i \rightarrow \infty$, onde $i$ representa o número de iterações, temos que,

$$
\theta^{(i)} \stackrel{\mathcal{D}}{\rightarrow} \theta, \quad \boldsymbol{w}^{(i)} \stackrel{\mathcal{D}}{\rightarrow} \boldsymbol{w},
$$

onde $\theta$ e $\boldsymbol{w}$ tem f.d.p. dadas por $p(\theta \mid \boldsymbol{y})$ e $p(\boldsymbol{w} \mid \boldsymbol{y})$, respectivamente. Desta maneira podemos utilizar os valores gerados de $\theta$, a partir de um tempo $t$ suficientemente grande, como uma amostra da distribuição a posteriori de interesse. Esse raciocínio é facilmente estendido para o caso de mais de um parâmetro, $\theta_{1}, \theta_{2}, . ., \theta_{s}$ e é simples verificar que os valores devem ser gerados iterativamente das seguintes distribuições condicionais

$$
\begin{aligned}
& \text { (a) } p\left(\theta_{i} \mid \theta_{j}, j \neq i, \boldsymbol{w}, \boldsymbol{y}\right), \forall i \mathrm{e} \\
& \text { (b) } p\left(\boldsymbol{w} \mid \theta_{i}, \forall i, \boldsymbol{y}\right) .
\end{aligned}
$$

Portanto, o DA é um algoritmo construido a partir das distribuições condicionais a posteriori, equivalente ao amostrador de Gibbs. Para mais detalhes sobre este algoritmo, incluindo observações sobre sua equivalência com o amostrador de Gibbs, veja o apêndice.

No problema de calibração o vetor aleatório que será observado é $\boldsymbol{y}_{*}=\left(y_{0}, y_{1}, \ldots, y_{n}\right)^{t} \mathrm{e}$ $\boldsymbol{\theta}=\left(\boldsymbol{\beta}^{t}, x_{0}\right)^{t}$. Utilizando a sugestão de Albert and Chib (1993), introduzimos no problema as seguintes variáveis latentes

$$
w_{i j}=\beta_{1}+\beta_{2} x_{i}+e_{i j}, \operatorname{com} e_{i j} \stackrel{i . i . d .}{\sim} \operatorname{El}\left(0,1 ; g_{i}\right), i=0, . ., n, j=1, . ., k_{i} .
$$

Se definirmos,

$$
z_{i j}= \begin{cases}1 & \text { se }, \quad w_{i j}>0 \\ 0 & \text { se, }, \quad w_{i j} \leq 0\end{cases}
$$


e $y_{i}=\sum_{j=1}^{k_{i}} z_{i j}$ temos que, $y_{i} \stackrel{i n d}{\sim} B\left(k_{i}, p_{i}\right)$ com $p_{i}=F\left(\beta_{1}+\beta_{2} x_{i}\right)$. De fato, como $p_{i}=$ $\operatorname{Prob}\left(z_{i j}=1\right)=\operatorname{Prob}\left(w_{i j}>0\right)$, então de 5.7 e considerando a simetria da f.d.a. $F$, temos que

$$
p_{i}=1-F\left(-\beta_{1}-\beta_{2} x_{i}\right)=F\left(\beta_{1}+\beta_{2} x_{i}\right) .
$$

Denotamos por $\boldsymbol{w}_{(i)}=\left(w_{i 1}, \ldots, w_{i k_{i}}\right)$ e $\boldsymbol{w}_{*}=\left(w_{(0)}, \ldots, w_{(n)}\right)$. Então, a densidade de probabilidades conjunta de $\left(\boldsymbol{y}_{*}, \boldsymbol{w}_{*}\right)$ condicional a $\boldsymbol{\beta}$ e $x_{0}$ pode ser obtida a partir da relação

$$
p\left(\boldsymbol{y}_{*}, \boldsymbol{w}_{*} \mid \boldsymbol{\beta}, \boldsymbol{x}, x_{0}\right)=p\left(\boldsymbol{y}_{*} \mid \boldsymbol{w}_{*}, \boldsymbol{\beta}, \boldsymbol{x}, x_{0}\right) p\left(\boldsymbol{w}_{*} \mid \boldsymbol{\beta}, \boldsymbol{x}, x_{0}\right) .
$$

Mas, pela construção dos $y_{i}^{\prime} s, p\left(\boldsymbol{y}_{*} \mid \boldsymbol{w}_{*}, \boldsymbol{\beta}, \boldsymbol{x}, x_{0}\right)=p\left(\boldsymbol{y}_{*} \mid \boldsymbol{w}_{*}\right)$, isto é, condicionalmente a $\boldsymbol{w}_{*}, \boldsymbol{y}_{*}$ é independente de $\boldsymbol{\beta}$ e $x_{0}$. Sem perda de generalidade, vamos considerar que cada vetor $\boldsymbol{w}_{(i)}$ tem seus componentes ordenados de forma decrescente, de modo que, fixado $y_{i}$, as primeiras $y_{i}$ posições do vetor $\boldsymbol{w}_{(i)}$ estão ocupadas por valores $w_{i j}>0$ e as restantes $\left(k_{i}-y_{i}\right)$ posições, por valores $w_{i j} \leq 0$. Deste modo, a. seguinte distribuição de probabilidades é obtida.

$$
p\left(y_{i} \mid \boldsymbol{w}_{(i)}\right)=\prod_{j=1}^{y_{i}} I_{\left(w_{i j}>0\right)} \prod_{j=y_{i}+1}^{k_{i}} I_{\left(w_{i j} \leq 0\right)} \quad i=0,1, \ldots, n
$$

Portanto,

$$
p\left(\boldsymbol{y}_{*} \mid \boldsymbol{w}_{*}\right)=\prod_{i=0}^{n} \prod_{j=1}^{y_{i}} I_{\left(w_{i j}>0\right)} \prod_{j=y_{i}+1}^{k_{i}} I_{\left(w_{i j} \leq 0\right)} .
$$

Além disso,

$$
p\left(\boldsymbol{w}_{*} \mid \boldsymbol{\beta}, \boldsymbol{x}, x_{0}\right)=\prod_{i=0}^{n} \prod_{j=1}^{k_{i}} g_{i}\left[\left(w_{i j}-\beta_{1}-\beta_{2} x_{i}\right)^{2}\right] .
$$

Finalmente, substituindo-se as duas últimas expressões em 5.8, resulta $p\left(\boldsymbol{y}_{*}, \boldsymbol{w}_{*} \mid \boldsymbol{\beta}, \boldsymbol{x}, x_{0}\right)=\prod_{i=0}^{n} \prod_{j=1}^{y_{i}} g_{i}\left[\left(w_{i j}-\beta_{1}-\beta_{2} x_{i}\right)^{2}\right] I_{\left(w_{i}>0\right)} \prod_{j=y_{i}+1}^{k_{i}} g_{i}\left[\left(w_{i j}-\beta_{1}-\beta_{2} x_{i}\right)^{2}\right] I_{\left(w_{i}, \leq 0\right)}$.

No que segue, vamos nos limitar a trabalhar com as distribuições elípticas representáveis. Neste caso, podemos escrever 


$$
g_{i}\left[\left(w_{i j}-\beta_{1}-\beta_{2} x_{i}\right)^{2}\right]=\int_{0}^{\infty}\left(2 \pi \lambda_{i}\right)^{-\frac{1}{2}} e^{-\frac{\left(w_{i j}-\beta_{1}-\beta_{2} x_{i}\right)^{2}}{2 \lambda_{i}}} d G\left(\lambda_{i}\right), \quad i=0, \ldots, n, j=1, \ldots, k_{i} .
$$

Acrescentando ao modelo um novo conjunto de parâmetros, $\lambda_{*}=\left(\lambda_{0}, \lambda_{1}, \ldots, \lambda_{n}\right)$, obtemos a seguinte expressão para o modelo condicional aumentado, $p\left(\boldsymbol{y}_{*}, \boldsymbol{w}_{*} \mid \boldsymbol{\beta}, \boldsymbol{x}, x_{0}, \boldsymbol{\lambda}_{*}\right)$,

$$
\prod_{i=0}^{n} \prod_{j=1}^{y_{i}}\left(2 \pi \lambda_{i}\right)^{-\frac{1}{2}} e^{-\frac{\left(w_{i j}-\beta_{1}-\beta_{2} x_{i}\right)^{2}}{2 \lambda_{i}}} I_{\left(w_{i j}>0\right)} \prod_{j=y_{i}+1}^{k_{i}}\left(2 \pi \lambda_{i}\right)^{-\frac{1}{2}} e^{-\frac{\left(w_{i j}-\beta_{1}-\beta_{2} x_{i}\right)^{2}}{2 \lambda_{i}}} I_{\left(w_{i j} \leq 0\right)} .
$$

A função de verossimilhança para os parâmetros $\boldsymbol{\beta}, x_{0}$ e $\boldsymbol{\lambda}_{*}$, que considera as observações $\left(\boldsymbol{y}_{*}, \boldsymbol{w}_{*}\right)$ é denominada verossimilhança aumentada e é dada por

$$
V_{\boldsymbol{y}_{*},} \boldsymbol{w}_{*}\left(\boldsymbol{\beta}, \boldsymbol{\lambda}^{*}, x_{0}\right) \propto\left[\prod_{i=0}^{n} \lambda_{i}^{-\frac{k_{i}}{2}} \prod_{j=1}^{y_{i}} I_{\left(w_{i j}>0\right)} \prod_{j=y_{i}+1}^{k_{i}} I_{\left(w_{i j} \leq 0\right)}\right] e^{-\frac{1}{2} \sum_{i=0}^{n} \sum_{j=1}^{k_{i}} \frac{\left(w_{i j}-\beta_{1}-\beta_{2} x_{i}\right)^{2}}{\lambda_{i}}} .
$$

De acordo com 5.6, para a implementação do algoritmo DA necessitamos das seguintes distribuições condicionais:

(i) $p\left(\boldsymbol{\beta} \mid x_{0}, \boldsymbol{\lambda}_{*}, \boldsymbol{w}_{*}, \boldsymbol{y}_{*}\right)$

(ii) $p\left(x_{0} \mid \boldsymbol{\beta}, \boldsymbol{\lambda}_{*}, \boldsymbol{w}_{*}, \boldsymbol{y}_{*}\right)$;

(iii) $p\left(\boldsymbol{\lambda}_{*} \mid \boldsymbol{\beta}, x_{0}, \boldsymbol{w}_{*}, \boldsymbol{y}_{*}\right) \mathrm{e}$

(iv) $p\left(\boldsymbol{w}_{*} \mid \boldsymbol{\beta}, x_{0}, \boldsymbol{\lambda}_{*}, \boldsymbol{y}_{*}\right)$.

As três primeiras estão associadas ao item $(a)$ de 5.6 , identificando $\theta=\left(\boldsymbol{\beta}, x_{0}, \boldsymbol{\lambda}_{*}\right)$.

Com a objetivo de obtermos formas conhecidas para as densidades acima, vamos considerar as seguintes hipóteses a priori:

$\boldsymbol{\beta}, \boldsymbol{\lambda}^{*}$ e $x_{0}$ são independentes,

$$
\boldsymbol{\beta} \sim N_{2}\left(\boldsymbol{b}_{0}, \boldsymbol{M}_{0}\right), \quad x_{0} \sim N\left(m_{0}, v_{0}\right),
$$

$\lambda_{0}, \lambda_{1}, \ldots, \lambda_{n}$ são i.i.d. com distribuição $G$, tal que $G(0)=0$. 
Utilizamos a notação:

$$
\begin{gathered}
\hat{x}=\frac{1}{n} \sum_{i=0}^{n} \frac{k_{i} x_{i}}{\lambda_{i}}, \quad \hat{w}=\frac{1}{n} \sum_{i=0}^{n} \frac{k_{i} \bar{w}_{i}}{\lambda_{i}}, \bar{w}_{i}=\frac{1}{k_{i}} \sum_{j=1}^{k_{i}} w_{i j}, \\
S_{x x}^{\lambda}=\sum_{i=0}^{n} \frac{k_{i}\left(x_{i}-\hat{x}\right)^{2}}{\lambda_{i}} \text { e } S_{x w}^{\lambda}=\sum_{i=0}^{n} \frac{k_{i} \hat{w}_{i}\left(x_{i}-\hat{x}\right)}{\lambda_{i}} .
\end{gathered}
$$

Além disso, $\hat{\beta}_{1}=\hat{w}-\hat{x} \hat{\beta}_{2}$ e $\hat{\beta}_{2}=S_{x w} / S_{x x}$ são os estimadores de máxima verossimilhança; e a notação $N T_{E}\left(\mu, \sigma^{2}\right)\left[N T_{D}\left(\mu, \sigma^{2}\right)\right]$ será utilizada para representar uma distribuição normal,com média $\mu$ e variância $\sigma^{2}$, truncada a esquerda [direita] do zero.

Proposição 5.1 Sob as condiçôes 5.9 e 5.10 obtemos as seguintes distribuições a posteriori condicionais

(i) $\boldsymbol{\beta} \mid x_{0}, \boldsymbol{\lambda}_{*}, \boldsymbol{w}_{*}, \boldsymbol{y}_{*} \sim N_{2}\left(\boldsymbol{b}_{1}, \boldsymbol{M}_{1}\right)$

(ii) $x_{0} \mid \boldsymbol{\beta}, \boldsymbol{\lambda}_{*}, \boldsymbol{w}_{*}, \boldsymbol{y}_{*} \sim N\left(m_{1}, v_{1}\right)$

(iii) $w_{i j} \mid \boldsymbol{\beta}, x_{0}, \boldsymbol{\lambda}_{*}, \boldsymbol{y}_{*}^{\text {ind }} \sim N T_{E}\left(\beta_{1}+\beta_{2} x_{i}, 1\right), \operatorname{para} j=1,2, \ldots, y_{i} e$ $w_{i j} \mid \boldsymbol{\beta}, x_{0}, \boldsymbol{\lambda}_{*}, \boldsymbol{y}_{*} \stackrel{i n d}{\sim} N T_{D}\left(\beta_{1}+\beta_{2} x_{i}, 1\right)$, para $j=y_{i}+1, \ldots, k_{i}$,

onde

$$
\begin{aligned}
& M_{1}=\left(M_{0}^{-1}+M^{-1}\right)^{-1}, \quad b_{1}=M_{1}\left(M_{0}^{-1} b_{0}+M^{-1} \hat{\boldsymbol{\beta}}\right), \\
& \hat{\boldsymbol{\beta}}=\left(\hat{\beta}_{1}, \hat{\beta}_{2}\right)^{t}, \quad \boldsymbol{M}=\left[\begin{array}{cc}
\left(\sum_{i=0}^{n} \frac{k_{i}}{\lambda_{i}}\right)^{-1}+\frac{\hat{x}^{2}}{S_{x x}^{\lambda}} & \frac{-\hat{x}}{S_{x x}^{\lambda}} \\
\frac{-\hat{x}}{S_{x x}^{\lambda}} & \frac{1}{S_{x x}^{\lambda}},
\end{array}\right], \\
& v_{1}=\left(v_{0}^{-1}+\frac{k_{0} \beta_{2}^{2}}{\lambda_{0}}\right)^{-1} \quad e \quad m_{1}=v_{1}\left[\frac{m_{0}}{v_{0}}+\frac{k_{0} \beta_{2}\left(\bar{w}_{0}-\beta_{1}\right)}{\lambda_{0}}\right]
\end{aligned}
$$

\section{Prova:}

Os resultados dos itens (ii) e (iii) são obtidos diretamente de 5.9 e 5.10 , utlizando-se a fórmula de Bayes. De fato,

$$
\begin{gathered}
p\left(x_{0} \mid \boldsymbol{\beta}, \boldsymbol{\lambda}_{*}, \boldsymbol{w}_{*}, \boldsymbol{y}_{*}\right) \propto e^{\frac{-1}{2}\left[\frac{k_{0}}{\lambda_{0}}\left(\bar{w}_{0}-\beta_{1}-\beta_{2} x_{0}\right)^{2}+\frac{1}{v_{0}}\left(x_{0}-m_{0}\right)^{2}\right]} \propto \\
\propto e^{\frac{-1}{2}\left[\frac{k_{0} \beta_{2}^{2}}{\lambda_{0}}\left(x_{0}-\frac{\bar{w}_{0}-\beta_{1}}{\beta_{2}}\right)^{2}+\frac{1}{v_{0}}\left(x_{0}-m_{0}\right)^{2}\right]},
\end{gathered}
$$


resultando em (ii). E ainda,

$$
\begin{gathered}
p\left(w_{i j} \mid \boldsymbol{\beta}, x_{0}, \boldsymbol{\lambda}_{*}, \boldsymbol{y}_{*}\right) \propto I_{\left(w_{i j}>0\right)} e^{\frac{-\left(w_{i j}-\beta_{1}-\beta_{2} x_{i}\right)^{2}}{2 \lambda_{i}}} \quad i=0,1, \ldots, n, j=1,2, \ldots, y_{i} \mathrm{e} \\
p\left(w_{i j} \mid \boldsymbol{\beta}, x_{0}, \boldsymbol{\lambda}_{*}, \boldsymbol{y}_{*}\right) \propto I_{\left(w_{i j} \leq 0\right)} e^{\frac{-\left(w_{i j}-\beta_{1}-\beta_{2} x_{i}\right)^{2}}{2 \lambda_{i}}} \quad i=0,1, \ldots, n, \quad j=y_{i}+1, \ldots, k_{i},
\end{gathered}
$$

obtendo-se (iii).

Para obtermos o resultado (i) vamos considerar a seguinte transformação de variáveis $\eta=\beta_{1}+\beta_{2} \hat{x}$, onde $\hat{x}$ foi definido anteriormente como uma média ponderada dos $x_{i}^{\prime} s$. Supondo conhecidos $x_{0}$ e $\boldsymbol{\lambda}_{*}$, a função de verossimilhança condicional a $x_{0}$ e $\boldsymbol{\lambda}_{*}$, considerando como parâmetros $\eta$ e $\beta_{2}$ é dada por

$$
V_{\boldsymbol{y}_{*}}, \boldsymbol{w}_{*}\left(\eta, \beta_{2}\right) \propto e^{\frac{-1}{2} \sum_{i=0}^{n} \frac{k_{i}}{\lambda_{i}}\left[\bar{w}_{i}-\eta-\beta_{2}\left(x_{i}-\hat{x}\right)\right]^{2}} .
$$

Após algumas simplificações temos,

$$
V_{\boldsymbol{y}_{*}, \boldsymbol{w}_{*}}\left(\eta, \beta_{2}\right) \propto e^{\frac{-1}{2}\left[\sum_{i=0}^{n} \frac{k_{i}}{\lambda_{i}}(\eta-\hat{w})^{2}+S_{\boldsymbol{x}}^{\lambda}\left(\beta_{2}-\hat{\beta}_{2}\right)^{2}\right]} .
$$

Portanto, se considerarmos uma distribuição a priori imprópria proporcional a uma constante $C$ para $\eta$ e $\beta_{2}$ as distribuições a posteriori de $\eta$ e $\beta_{2}$ são normais independentes com médias $\hat{w}, \hat{\beta}_{2}$ e variâncias $\left(\sum_{i=0}^{n} \frac{k_{i}}{\lambda_{i}}\right)^{-1},\left(S_{x x}^{\lambda}\right)^{-1}$, respectivamente. Utilizando este fato e as propriedades da esperança, variância e covariância de uma variável aleatória, obtemos

$$
\begin{aligned}
& E\left[\beta_{1} \mid x_{0}, \boldsymbol{\lambda}_{*}, \boldsymbol{w}_{*}, \boldsymbol{y}_{*}\right]=\hat{w}-\hat{x} \hat{\beta}_{2}, \\
& V\left[\beta_{1} \mid x_{0}, \boldsymbol{\lambda}_{*}, \boldsymbol{w}_{*}, \boldsymbol{y}_{*}\right]=\left[\sum_{i=0}^{n} \frac{k_{i}}{\lambda_{i}}\right]^{-1}+\frac{\hat{x}^{2}}{S_{x x}^{\lambda}} \mathrm{e} \\
& \operatorname{Cov}\left[\beta_{1}, \beta_{2} \mid x_{0}, \boldsymbol{\lambda}_{*}, \boldsymbol{w}_{*}, \boldsymbol{y}_{*}\right]=\frac{\hat{x}}{S_{x x}^{\lambda}} .
\end{aligned}
$$

Além disso, se $\left(\eta, \beta_{2}\right)$ tem distribuição normal bivariada, então $\left(\beta_{1}, \beta_{2}\right)=\left(\eta-\hat{x} \beta_{2}, \beta_{2}\right)$ também é normal bivariada, e ainda a escolha de distribuições a priori impróprias para $\eta \mathrm{e}$ $\beta_{2}$ implica que a distribuição a priori para $\beta_{1}$ também deve ser imprópria (proporcional a uma constante). Portanto, com a suposição de distribuições impróprias para $\beta_{1}$ e $\beta_{2}$, temos que a distribuição a posteriori de $\left(\beta_{1}, \beta_{2}\right)$ condicional a $x_{0}, \lambda^{*}$ e $w^{*}$, é normal bivariada com vetor de médias 


$$
\left[\begin{array}{c}
\hat{w}-\hat{x} \hat{\beta}_{2} \\
\hat{\beta}_{2}
\end{array}\right]
$$

e matriz de covariância

$$
\left[\begin{array}{cc}
\left(\sum_{i=0}^{n} \frac{k_{i}}{\lambda_{i}}\right)^{-1}+\frac{\hat{x}^{2}}{S_{x x}^{\lambda}} & -\frac{\hat{x}}{S_{x x}^{\lambda}} \\
-\frac{\hat{x}}{S_{x x}^{\lambda}} & \frac{1}{S_{x x}^{\lambda}}
\end{array}\right] .
$$

Finalmente para a obtenção do resultado $(i)$ basta considerar a distribuição a priori para $\left(\beta_{1}, \beta_{2}\right)$ dada em 5.10 e as simplificações usuais feitas para classe conjugada da normal multivariada (Press 1984).

O modelo probit é um caso particular onde supomos $G$ degenerada no ponto 1, resultando que $F$ é normal. Neste caso, as expressões da proposição 3.3 ficam simplificadas fazendo $\lambda^{*}=(1,1, . ., 1)^{t}$. Para aplicação do resultado ao modelo logit, vamos considerar o fato de que a distribuição t-Student propicia uma boa aproximação para a distribuição logística, já que esta última não é representável (Kano 1994). Abaixo apresentamos uma ilustração em forma gráfica dessa aproximação. A figura 5.1 relaciona os quantis da distribuição logística com os de uma t-Student para alguns valores de graus de liberdades, $\nu=4,8,16$. Observamos que a melhor aproximação ocorre quando $\nu=8$.

Figura 5.1: Gráfico dos quantis da logística x t-Student (probabilidades entre 0.001 e 0.999 )

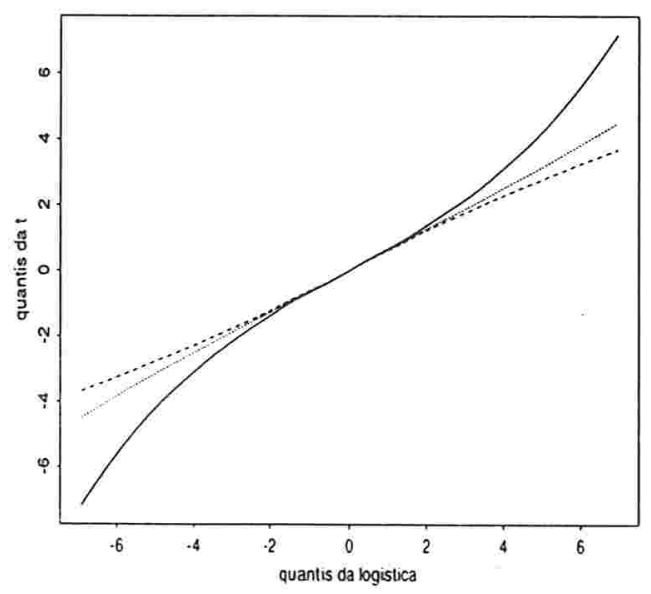

Linha contínua para $\nu=4$, linha pontilhada para $\nu=8$ e linha tracejada para $\nu=16$. 
Portanto, ao considerarmos $w_{i j} \mid \boldsymbol{\beta}, \nu, x_{i} \sim T\left(\beta_{1}+\beta_{2} x_{i}, 1 ; \nu\right), i=0,1, \ldots, n ; j=$ $1,2, . ., k_{i}$, estamos trabalhando com uma aproximação do modelo logit. Entretanto, sabemos que essa suposição é equivalente a considerar de forma hierárquica as seguintes suposições:

$$
\begin{aligned}
& \text { (i) } w_{i j} \mid \boldsymbol{\beta}, \nu, x_{i}, \lambda_{i} \stackrel{i n d}{\sim} N\left(\beta_{1}+\beta_{2} x_{i}, \lambda_{i}\right), \\
& \text { (ii) } \lambda_{i} \mid \nu \sim I G\left(\frac{\nu}{2}, \frac{\nu}{2}\right) i=0,1, \ldots, n ; j=1,2, . ., k_{i} .
\end{aligned}
$$

Neste caso é possível obter uma forma explicita para a distribuição a posteriori de $\lambda_{*}$, condicional aos demais parâmetros.

Proposição 5.2 Sob as condições 5.9, 5.10 e sendo $G$ a f.d.a. de uma distribuição $I G\left(\frac{\nu}{2}, \frac{\nu}{2}\right)$, temos que

$$
\lambda_{i} \mid \boldsymbol{\beta}, x_{0}, \nu, \boldsymbol{w}_{*}, \boldsymbol{y}_{*}^{i n d} \sim I G\left(\frac{\nu_{1}}{2}, \frac{s_{i}}{2}\right), \quad i=0,1, . ., n
$$

com,

$$
\nu_{1}=\nu+1 \quad \text { e } \quad s_{i}=\nu+\sum_{j=1}^{k_{i}}\left(w_{i j}-\beta_{1}-\beta_{2} x_{i}\right)^{2} .
$$

Prova: Utilizando-se a fórmula de Bayes o resultado é imediato de 5.9. De fato,

$$
p\left(\boldsymbol{\lambda}^{*} \mid \boldsymbol{\beta}, x_{0}, \nu, \boldsymbol{w}_{*}, \boldsymbol{y}_{*}\right) \propto \prod_{i=0}^{n} \lambda_{i}^{-\frac{(\nu+1)}{2}} e^{-\frac{1}{\lambda_{i}}\left[\nu+\sum_{j=1}^{k_{i}}\left(w_{i j}-\beta_{1}-\beta_{2} x_{i}\right)^{2}\right]},
$$

o que implica que a distribuição conjunta de $\lambda^{*}$ é um produto de distribuições gamasinvertidas. $\diamond$

Os resultados das proposições 5.3 e 5.4 nos permite implementar o algoritmo DA para os modelos logit e probit e assim obtermos a distribuição a posteriori marginal de $x_{0}$. Estes resultados serão úteis para a aplicação feita na seção 5.4 . 


\subsubsection{Aproximação assintótica para a distribuição a posteriori}

Sabemos que sob certas condições de regularidade a distribuição a posteriori pode ser aproximada por uma distribuição normal cuja média é o estimador de máxima verossimilhança e a matriz de covariância é a inversa da matriz de informação de Fisher avaliada no ponto de máxima verossimilhança (Press 1984).

A seguir vamos determinar os estimadores de máxima verossimilhança e a matriz de informação de Fisher associados ao problema de calibração sob o modelo 5.2. Para tal, trabalhamos com o logaritmo da função de verossimilhança, dada em 5.3, que denotaremos por $L($.$) . Assim,$

$$
L\left(\boldsymbol{\beta}, x_{0}\right)=\sum_{i=0}^{n} \ln \left(\begin{array}{c}
k_{i} \\
y_{i}
\end{array}\right)+\sum_{i=0}^{n} y_{i} \ln \left[\frac{F\left(\beta_{1}+\beta_{2} x_{i}\right)}{1-F\left(\beta_{1}+\beta_{2} x_{i}\right)}\right]+\sum_{i=0}^{n} k_{i} \ln \left[1-F\left(\beta_{1}+\beta_{2} x_{i}\right)\right] .
$$

A propriedade de invariância dos estimadores de máxima verossimilhança (Mood et al. 1974) nos permite derivar o estimador de máxima verossimilhança de $x_{0}$ em função dos estimadores de máxima verossimilhança de $\beta_{1}$ e $\beta_{2}$. Por hipótese $p_{0}=F\left(\beta_{1}+\beta_{2} x_{0}\right)$ e portanto, $x_{0}=\left(F^{-1}\left(p_{0}\right)-\beta_{1}\right) / \beta_{2}$. Considerando que $y_{0} / k_{0}, \hat{\beta}_{1}$ e $\hat{\beta}_{2}$ são os estimadores de máxima verossimilhança de $p_{0}, \beta_{1}$ e $\beta_{2}$, respectivamente, pela propriedade de invariância temos que o e.m.v. para $x_{0}$ é

$$
\hat{x}_{0}=\frac{F^{-1}\left(y_{0} / k_{0}\right)-\hat{\beta}_{1}}{\hat{\beta}_{2}} .
$$

Deste modo, temos que

(i) no modelo probit $\hat{x}_{0}=\left(a_{0}-\hat{\beta}_{1}\right) / \hat{\beta}_{2}$, onde $a_{0}$ é tal que, $P\left(Z \leq a_{0}\right)=y_{0} / k_{0}$,

$$
Z \sim N(0,1) \mathrm{e}
$$

(ii) no modelo logit $\hat{x}_{0}=\left(\log \frac{y_{0}}{k_{0}-y_{0}}-\hat{\beta}_{1}\right) / \hat{\beta}_{2}$.

Como já foi mencionado anteriormente, é conhecido, da teoria de modelos lineares generalizados(MLG) que os estimadores de máxima verossimilhança de $\beta_{1}$ e $\beta_{2}$ não tem formas explicitas e algoritmos numéricos, como o método de Newton-Raphson, são utilizados para obtê-los. Essas estimativas poderão ser utilizadas para a obtenção da estimativa de máxima verossimilhaça do problema de calibração. 
Proposição 5.3 A matriz de informação de Fisher para o modelo 5.2 é dada por

$$
I(\theta)=\left[\begin{array}{ccc}
\sum_{i=0}^{n} k_{i} w_{i} & \sum_{i=0}^{n} k_{i} x_{i} w_{i} & \beta_{2} k_{0} w_{0} \\
\sum_{i=0}^{n} k_{i} x_{i} w_{i} & \sum_{i=0}^{n} k_{i} x_{i}^{2} w_{i} & \beta_{2} k_{0} x_{0} w_{0} \\
\beta_{2} k_{0} w_{0} & \beta_{2} k_{0} x_{0} w_{0} & \beta_{2}^{2} k_{0} w_{0}
\end{array}\right]
$$

onde

$$
w_{i}=\frac{f^{2}\left(\beta_{1}+\beta_{2} x_{i}\right)}{F\left(\beta_{1}+\beta_{2} x_{i}\right)\left[1-F\left(\beta_{1}+\beta_{2} x_{i}\right)\right]}, i=0,1, . ., n
$$

e $f$ é a função densidade de probabilidades associada a distribuição $F$.

\section{Prova:}

As derivadas de primeira ordem do logaritmo da função de verossimilhança, 5.12 , são

$$
\begin{gathered}
\frac{\partial L}{\partial \beta_{1}}=\sum_{i=0}^{n} \frac{f\left(\beta_{1}+\beta_{2} x_{i}\right)\left[y_{i}-k_{i} F\left(\beta_{1}+\beta_{2} x_{i}\right)\right]}{F\left(\beta_{1}+\beta_{2} x_{i}\right)\left[1-F\left(\beta_{1}+\beta_{2} x_{i}\right)\right]}, \\
\frac{\partial L}{\partial \beta_{2}}=\sum_{i=0}^{n} \frac{f\left(\beta_{1}+\beta_{2} x_{i}\right)\left[x_{i} y_{i}-k_{i} x_{i} F\left(\beta_{1}+\beta_{2} x_{i}\right)\right]}{F\left(\beta_{1}+\beta_{2} x_{i}\right)\left[1-F\left(\beta_{1}+\beta_{2} x_{i}\right)\right]} \mathrm{e} \\
\frac{\partial L}{\partial x_{0}}=\frac{\beta_{2} f\left(\beta_{1}+\beta_{2} x_{0}\right)\left[x_{0} y_{0}-k_{0} x_{0} F\left(\beta_{1}+\beta_{2} x_{0}\right)\right]}{F\left(\beta_{1}+\beta_{2} x_{0}\right)\left[1-F\left(\beta_{1}+\beta_{2} x_{0}\right)\right]} .
\end{gathered}
$$

Utilizando a notação

$$
\begin{aligned}
r_{i}=\frac{\left[y_{i}-k_{i} F\left(\beta_{1}+\beta_{2} x_{i}\right)\right]}{F\left(\beta_{1}+\beta_{2} x_{i}\right)\left[1-F\left(\beta_{1}+\beta x_{i}\right)\right]}\left[f^{\prime}\left(\beta_{1}+\beta x_{i}\right)+\right. \\
\left.\quad \frac{f^{2}\left(\beta_{1}+\beta x_{i}\right)}{F\left(\beta_{1}+\beta x_{i}\right)\left[1-F\left(\beta_{1}+\beta x_{i}\right)\right]}\left[2 F\left(\beta_{1}+\beta x_{i}\right)-1\right]\right],
\end{aligned}
$$

onde $f^{\prime}(u)=\frac{\partial f(u)}{\partial u}$, temos as seguintes expressões para as derivadas de segunda ordem

$$
\begin{array}{ll}
\frac{\partial^{2} L}{\partial^{2} \beta_{1}}=\sum_{i=0}^{n} r_{i}-\sum_{i=0}^{n} k_{i} w_{i}, & \frac{\partial^{2} L}{\partial \beta_{1} \partial \beta_{2}}=\sum_{i=0}^{n} x_{i} r_{i}-\sum_{i=0}^{n} k_{i} x_{i} w_{i}, \\
\frac{\partial^{2} L}{\partial \beta_{1} \partial x_{0}}=\beta_{2} r_{0}-\beta_{2} k_{0} w_{0}, & \frac{\partial^{2} L}{\partial^{2} \beta_{2}}=\sum_{i=0}^{n} x_{i}^{2} r_{i}-\sum_{i=0}^{n} k_{i} x_{i}^{2} w_{i}, \\
\frac{\partial^{2} L}{\partial \beta_{2} \partial x_{0}}=\beta_{2} x_{0} r_{0}-\beta_{2} x_{0} k_{0} w_{0} \text { e } & \frac{\partial^{2} L}{\partial^{2} x_{0}}=\beta_{2}^{2} r_{0}-\beta_{2}^{2} k_{0} w_{0} .
\end{array}
$$


Mas, como $E\left[y_{i}\right]=k_{i} F\left(\beta_{1}+\beta_{2} x_{i}\right)$, então $E\left[r_{i}\right]=0, \forall i$. Portanto,

$$
\begin{aligned}
& E\left[-\frac{\partial^{2} L}{\partial^{2} \beta_{1}}\right]=\sum_{i=0}^{n} k_{i} w_{i}, \quad E\left[-\frac{\partial^{2} L}{\partial \beta_{1} \partial \beta_{2}}\right]=\sum_{i=0}^{n} k_{i} x_{i} w_{i}, \quad E\left[-\frac{\partial^{2} L}{\partial \beta_{1} \partial x_{0}}\right]=\beta_{2} k_{0} w_{0}, \\
& E\left[\frac{\partial^{2} L}{\partial^{2} \beta_{2}}\right]=\sum_{i=0}^{n} k_{i} x_{i}^{2} w_{i}, \quad E\left[-\frac{\partial^{2} L}{\partial \beta_{2} \partial x_{0}}\right]=\beta x_{0} k_{0} w_{0} \text { e } E\left[-\frac{\partial^{2} L}{\partial^{2} x_{0}}\right]=\beta_{2}^{2} k_{0} w_{0} . \diamond
\end{aligned}
$$

Notamos que no modelo logit $f(t)=F(t)[1-F(t)]$, de onde segue que

$$
w_{i}=\frac{f^{2}\left(\beta_{1}+\beta_{2} x_{i}\right)}{F\left(\beta_{1}+\beta_{2} x_{i}\right)\left[1-F\left(\beta_{1}+\beta_{2} x_{i}\right)\right]}=f\left(\beta_{1}+\beta_{2} x_{i}\right), i=0,1, \ldots, n .
$$

No que segue, denotamos por $I(\hat{\theta})$ a matriz de informação de Fisher no ponto de máxima verossimilhança, $\hat{\theta}=\left(\hat{\beta}_{1}, \hat{\beta}_{2}, \hat{x}_{0}\right)^{t}$. Assim, para determinar $I(\hat{\theta})$ basta substituir os $w_{i}^{\prime} s$ de $I(\theta)$ por

$$
\begin{gathered}
\hat{w}_{i}=\frac{f^{2}\left(\hat{\beta}_{1}+\hat{\beta}_{2} x_{i}\right)}{F\left(\hat{\beta}_{1}+\hat{\beta}_{2} x_{i}\right)\left[1-F\left(\hat{\beta}_{1}+\hat{\beta}_{2} x_{i}\right)\right]}, i=1, . ., n \mathrm{e} \\
\hat{w}_{0}=\frac{f^{2}\left(\hat{\beta}_{1}+\hat{\beta}_{2} \hat{x}_{0}\right)}{F\left(\hat{\beta}_{1}+\hat{\beta}_{2} \hat{x}_{0}\right)\left[1-F\left(\hat{\beta}_{1}+\hat{\beta}_{2} \hat{x}_{0}\right)\right]} .
\end{gathered}
$$

Se as condições de regularidades para a convergência da distribuição a posteriori forem satisfeitas, então podemos utilizar a distribuição normal $N_{3}\left(\hat{\boldsymbol{\theta}},[\boldsymbol{I}(\hat{\boldsymbol{\theta}})]^{-1}\right)$, como aproximação da distribuição a posteriori conjunta de $\left(\beta_{1}, \beta_{2}, x_{0}\right)$, para $n$ suficientemente grande. Consequentemente, a distribuição a posteriori marginal de $x_{0}$ seria assintoticamente normal com média $\hat{x}_{0}$ e variância $a_{33}$, onde $a_{33}$ é o elemento na posição $3 \times 3$ da matriz $[\boldsymbol{I}(\hat{\boldsymbol{\theta}})]^{-1}$. As condições usuais para a convergência da distribuição a posteriori (Press 1984) são estabelecidas sob a suposição de que os valores observados formam uma amostra i.i.d., o que não ocorre no nosso problema. Os valores $y_{0}, y_{1}, \ldots, y_{n}$ são condicionalmente independentes, mas não são identicamente distribuídos. Chen (1985) estabelece condições gerais para a convergência de uma distribuição a posteriori para uma normal. Entretanto, tais condições não são operacionais. Deixaremos a tentativa de verificação destas condições para uma trabalho futuro. Ainda assim, utilizamos a aproximação normal como uma possível estimativa da distribuição a posteriori desejada. No exemplo, da seção 5.4, os 
valores obtidos com esta aproximação serão comparados com as estimativas obtidas via algoritmo DA.

No modelo binomial outro tipo de convergência que pode ser investigado é quando os $k_{i}^{\prime} s$ crescem, mantendo-se fixo o $n$. Neste caso, sabemos que a distribuição binomial aproxima-se de uma distribuição normal, da seguinte maneira.

$$
\frac{y_{i}-k_{i} p_{i}}{k_{i} p_{i}\left(1-p_{i}\right)} \stackrel{\mathcal{D}}{\rightarrow} N(0,1)
$$

onde $x_{i} \stackrel{\mathcal{D}}{\rightarrow} N\left(\mu, \sigma^{2}\right)$ significa que a v.a. $x_{i}$ converge em distribuição para uma v.a. com distribuição $N\left(\mu, \sigma^{2}\right)$.

Neste caso, podemos pensar em trabalhar com a suposição de normalidade. Mais precisamente, supor que $y_{i}$ tem uma distribuição aproximadamente normal com média $k_{i} F\left(\beta_{1}+\beta_{2} x_{i}\right)$ e variância $k_{i} F\left(\beta_{1}+\beta_{2} x_{i}\right)\left[1-F\left(\beta_{1}+\beta_{2} x_{i}\right)\right]$. Recaímos num problema de calibração sob normalidade, mas perdemos a hipótese de linearidade entre $y$ e $x$. Para contornar este problema podemos utilizar uma transformação de variáveis. Vamos considerar $t_{i}=F^{-1}\left(y_{i} / k_{i}\right)$. Utilizando-se o método delta e considerando-se a condição 5.15 , encontramos a aproximação normal adequada para a nova variável $t_{i}$, dada por

$$
\left(k_{i}\right)^{\frac{1}{2}}\left[t_{i}-\beta_{1}-\beta_{2} x_{i}\right] \stackrel{\mathcal{D}}{\rightarrow} N\left(0,\left\{g^{\prime}\left[F\left(\beta_{1}+\beta_{2} x_{i}\right)\right]\right\}^{2} F\left(\beta_{1}+\beta_{2} x_{i}\right)\left[1-F\left(\beta_{1}+\beta_{2} x_{i}\right)\right]\right)
$$

onde $g=F^{-1}$. Com este resultado recuperamos a linearidade, isto é, $T$ é linear em $x$. Entretanto, a variância dos erros depende de $x_{i}, \boldsymbol{\beta}$ e $F$.

\subsection{O modelo multinomial}

Considere o modelo

$$
\boldsymbol{y}_{i} \mid x_{i} \sim M_{p}\left(k_{i}, \boldsymbol{p}_{i}\right),
$$

onde $\boldsymbol{y}_{i}=\left(y_{i 1}, y_{i 2}, \ldots, y_{i q}\right)$ e $\boldsymbol{p}_{i}=\left(p_{i 1}, p_{i 2}, \ldots, p_{i q}\right)$ depende do valor de $x_{i}$.

Vamos considerar duas situações: quando as categorias são ordenadas e quando não é possível ordená-las. Na primeira situação a implementação do DA é obtida a partir de uma extensão do que foi desenvolvido para a binomial, onde as variáveis latentes consideradas são exatamente as mesmas do caso binomial. A alteração se dá com a introdução de um novo conjunto de parâmetros. No caso binomial a área sob a função densidade de 
probabilidade de $F$ foi particionada em duas partes: a primeira representando a probabilidade associada a categoria 1 (sucessso); e a segunda a probabilidade complementar. Para o caso multinomial devemos dividir a área total sob a densidade em $q$ partes, cada uma asssociada a probabilidade de uma das categorias. Estes pontos de cortes, serão os novos parâmetros do modelo. A segunda situação, quando as categorias não são ordenadas é um pouco mais complicada de ser tratada e um conjunto maior de variáveis latentes será considerado.

\subsubsection{Categorias ordenadas}

Com a hipótese de que as categorias são ordenadas uma extensão natural do modelo binomial é obtida considerando-se as mesmas variáveis latentes de 5.7 e introduzindo-se um novo conjunto de parâmetros $\gamma_{1}, \gamma_{2}, . ., \gamma_{q-1}$ de modo que

$$
z_{i j}=l, \quad \text { se } \quad \gamma_{l-1}<w_{i j}<\gamma_{l} \quad l=1,2, \ldots, q
$$

onde $\gamma_{0}=-\infty, \gamma_{q}=\infty$ e $\gamma_{1}<\gamma_{2}<\ldots<\gamma_{q-1}$. Considerando-se $y_{i l}$ como o número de vezes que $Z_{i j}=l$ para $j=1,2, . ., k_{i}$, temos que $\boldsymbol{y}_{i}=\left(y_{i 1}, \ldots, y_{i p}\right)$ tem distribuição multinomial dada em 5.16. Além disso, se $\eta_{i l}=\sum_{j=1}^{l} p_{i j}$ é a probabilidade acumulada na l-ésima categoria, então

$$
\eta_{i l}=F\left(\gamma_{l}-\beta_{1}-\beta_{2} x_{i}\right), \quad i=0,1, . ., n, \quad l=1,2, . . q-1,
$$

onde $F$ é uma distribuição elíptica representável. No caso de $F$ ser a distribuição normal padrão, $F=\Phi$, essa construção é a extensão do modelo probit dada por Aitchison and Silvey (1957).

Um novo conjunto de parâmetros foi introduzido no modelo, os $\gamma_{i}^{\prime} s$, que denotamos por $\gamma=\left(\gamma_{1}, \gamma_{2}, \ldots, \gamma_{q-1}\right)$. Utilizando um desenvolvimento análogo ao caso binomial, a função de verossimilhança para o modelo aumentado é dada por

$$
V_{\boldsymbol{y}}, \boldsymbol{w},\left(\boldsymbol{\beta}, \lambda^{*}, \gamma, x_{0}\right) \propto \prod_{i=0}^{n} \lambda_{i}^{-\frac{k_{i}}{2}}\left[\prod_{l=1}^{q} \prod_{j=t_{t_{(l-1)}+1}}^{t_{i l}} I_{\left(\gamma_{l-1}<w_{i}<\gamma_{l}\right)}\right] e^{-\frac{1}{2} \sum_{j=1}^{k_{i}} \frac{\left(w_{i j}-\beta_{1}-\beta x_{i}\right)^{2}}{\lambda_{i}}},
$$

onde $t_{i l}=\sum_{j=1}^{l} y_{i j} \quad l=1,2, . ., q$ e $t_{i 0}=0$. 
Considerando-se as distribuições a priori de 5.10 os resultados (i) e (ii) da proposição (5.3) são mantidos. Esses resultados não dependem do novo parâmetro $\gamma$. No entanto para as distribuições condicionais dos $w_{i j}^{\prime} s$ temos a seguinte alteração

$$
(i i i)^{\prime} w_{i j} \mid \boldsymbol{\beta}, x_{0}, \boldsymbol{\gamma}, \boldsymbol{\lambda}_{*}, \boldsymbol{y}_{*} \sim N T_{\left[\gamma_{l-1}, \gamma_{l}\right]}\left(\beta_{1}+\beta_{2} x_{i}, 1\right), \quad \text { para } j=t_{i(l-1)}+1, . ., t_{i l}
$$

onde, $N T_{[a, b]}\left(\mu, \sigma^{2}\right)$ representa uma distribuição normal com média $\mu$ e variância $\sigma^{2}$, truncada a esquerda de $a$ e a direita de $b, i=0,1, . ., n$ e $l=1,2, . . q$.

No caso particular de $\boldsymbol{\lambda}_{*}=(1,1, \ldots, 1)$ temos que $F=\Phi$ (modelo probit) e portanto, para implementação do algoritmo DA só resta obter a distribuição condicional a posteriori de $\gamma$.

Proposição 5.4 Considere 5.17 e as hipóteses a priori estabelecidas por 5.10. Se,

$$
\gamma_{l} \sim U_{\left[a_{l}, d_{l}\right)} \quad l=1,2, \ldots, q-1, \quad a_{l}<d_{l} \leq a_{l+1}
$$

então,

$$
\gamma_{l} \mid \boldsymbol{\beta}, x_{0}, \boldsymbol{w}_{*}, \boldsymbol{y}_{*} \sim U_{\left[A_{l}, D_{l}\right)} \text { independentes }
$$

onde

$$
\begin{aligned}
& A_{l}=\max \left(M_{l}, a_{l}\right) \text { e } D_{l}=\min \left(m_{l}, d_{l}\right), \\
& M_{l}=\max \left(w_{i j}\right), i=0,1, . ., n \text { e } j=t_{i(l-1)}+1, \ldots, t_{i l} e \\
& m_{l}=\min \left(w_{i j}\right), i=0,1, \ldots, n \text { e } j=t_{i l}+1, \ldots, t_{i(l+1)} .
\end{aligned}
$$

\section{Prova:}

Utilizando a fórmula de Bayes temos que

$$
p\left(\gamma_{l} \mid \boldsymbol{\beta}, \boldsymbol{w}_{*}, x_{0}, \boldsymbol{y}_{*}\right) \propto \prod_{i=0}^{n} \prod_{j=t_{i}(l-1)+1}^{t_{i l}} I_{\left(\gamma_{l-1}<W_{i j}<\gamma_{l}\right)} \prod_{j=t_{i l}+1}^{t_{i(l+1)}} I_{\left(\gamma_{l}<W_{i}<\gamma_{l+1}\right)} I_{\left(a_{l}<\gamma_{l}<d_{l}\right)}
$$

que é equivalente a

$$
p\left(\gamma_{l} \mid \boldsymbol{\beta}, \boldsymbol{w}^{*}, x_{0}, y^{*}\right) \propto I_{\left(M<\gamma_{l}<m\right)} I_{\left(a_{l}<\gamma_{l}<d_{l}\right)}=I_{\left[A_{l} \leq \gamma_{l}<D_{l}\right)} . \diamond
$$




\subsubsection{Categorias não ordenadas}

Considere a situação onde as possíveis respostas formam um conjunto $\left\{c_{1}, c_{2}, . ., c_{q}\right\}$, sem que seja possível ordenar as categorias $c_{i}^{\prime} s$. A escolha das variáveis latentes para essa situação basea-se na extensão do modelo probit apresentada por Aitchison and Bennet (1970) e são tais que

$$
w_{i j l}=\alpha_{l}+\beta_{l} x_{i}+e_{i j l}
$$

onde $e_{i j l} \sim E l\left(0,1 ; g_{i}\right), \quad j=1,2, . ., k_{i}, l=1,2, . ., q$.

Novamente, vamos considerar somente o caso onde $g$ é representável.

Definindo $z_{i j}=l$, quando $w_{i j l}=\max _{r}\left(w_{i j r}\right)$ e $y_{i l}$ como o número de vezes que $z_{i j}=l$ para $j=1,2, \ldots, k_{i}$, temos que $\boldsymbol{y}_{i}=\left(y_{i 1}, \ldots, y_{i q}\right)$ tem distribuição $M_{q}\left(k_{i}, \boldsymbol{p}_{i}\right)$, onde $\boldsymbol{p}_{i}=$ $\left(p_{i 1}, \ldots, p_{i q}\right)$ é tal que

$$
p_{i l}=P\left(w_{i j l}=\max _{r}\left(w_{i j r}\right)\right)=P\left(w_{i j l}>w_{i j r}, \forall r, r \neq l\right) .
$$

Esse modelo coincide com o de Aitchison and Bennet (1970) quando a distribuição a priori de $\lambda^{*}$ á degenerada no ponto $(1,1, . ., 1)$.

Denotamos por $\boldsymbol{w}_{*}$ o vetor cujas componentes são os $w_{i j l}, \forall i, \forall j, \forall l$, e por $\boldsymbol{\alpha}=\left(\alpha_{1}, \ldots, \alpha_{q}\right)$ e $\boldsymbol{\beta}=\left(\beta_{1}, \ldots, \beta_{q}\right)$. Então, da distribuição conjunta de $\boldsymbol{y}_{*}=\left(\boldsymbol{y}_{0}, \ldots, \boldsymbol{y}_{n}\right)$ e $\boldsymbol{w}_{*}$, obtemos para função de verossimilhança aumentada a seguinte expressão

$$
V_{\boldsymbol{y}_{*}}, \boldsymbol{w}_{*}\left(\boldsymbol{\alpha}_{*}, \boldsymbol{\beta}_{*}, \boldsymbol{\lambda}_{*}, x_{0}\right) \propto \prod_{i=0}^{n} \lambda_{i}^{-\frac{q k_{i}}{2}}\left[\prod_{l=1}^{q} \prod_{j=t_{i(l-1)}+1}^{t_{i l}} I_{\left(w_{i j l}=m a x_{r} w_{i j r}\right)}\right] e^{-\frac{1}{2} \sum_{j=1}^{k_{i}} \sum_{r=1}^{q} \frac{\left(w_{i j r}-\alpha_{r}-\beta_{r} x_{i}\right)^{2}}{\lambda_{i}}}
$$

Vamos considerar as seguintes suposições a priori:

(i) $\left(\alpha_{1}, \beta_{1}\right), \ldots,\left(\alpha_{q}, \beta_{q}\right), \lambda^{*}$ e $x_{0}$ são independentes e

(ii) $\left(\alpha_{r}, \beta_{r}\right) \sim N_{2}\left(B_{r 0}, M_{r 0}\right), r=1,2, . ., q$, e $x_{0} \sim N\left(m_{0}, v_{0}\right)$ 
Proposição 5.5 Sob as condições 5.20 e 5.21 obtemos as seguintes distribuiçôes a posteriori condicionais

(i) $\left(\alpha_{l}, \beta_{l}\right) \mid x_{0}, \boldsymbol{\lambda}^{*}, \boldsymbol{w}_{*}, \boldsymbol{y}_{*} \sim N_{2}\left(B_{l 1}, M_{l 1}\right)$,

(ii) $x_{0} \mid \boldsymbol{\alpha}_{*}, \boldsymbol{\beta}_{*}, \boldsymbol{\lambda}^{*}, \boldsymbol{w}_{*}, \boldsymbol{y}_{*} \sim N\left(m_{1}, v_{1}\right)$ e

(iii) $p\left(w_{i j 1}, w_{i j 2}, \ldots, w i j q \mid \boldsymbol{\alpha}_{*}, \boldsymbol{\beta}_{*}, x_{0}, \boldsymbol{\lambda}_{*}, \boldsymbol{y}_{*}\right) \propto I_{\left(w_{i j l}=\max _{r} w_{i j r}\right)} e^{-\frac{1}{2} \sum_{r=1}^{q} \frac{\left(w_{i j r}-\alpha_{r}-\beta_{r} x_{i}\right)^{2}}{\lambda_{i}}}$

para $j \in\left\{t_{i(l-1)}, \ldots, t_{i l}\right\} ; i=0,1, \ldots, n$ e $l=1,2, . ., q$.

Onde,

$$
\begin{aligned}
& M_{l 1}=\left(M_{0}^{-1}+M^{-1}\right)^{-1}, \quad B_{l 1}=M_{1}\left(M_{0}^{-1} B_{0}+M^{-1} B_{l}\right), \\
& B_{l}=\left[\begin{array}{c}
\hat{\alpha}_{l} \\
\hat{\beta}_{l}
\end{array}\right], \quad M=\left[\begin{array}{cc}
\left(\sum_{i=0}^{n} \frac{k_{i}}{\lambda_{i}}\right)^{-1}+\frac{\hat{x}^{2}}{S_{x x}^{\lambda}} & \frac{-\hat{x}}{S_{x x}^{\lambda}} \\
\frac{-\hat{x}}{S_{x x}^{\lambda}} & \frac{1}{S_{x x}^{\lambda}}
\end{array}\right], \\
& v_{1}=\left(v_{0}^{-1}+\frac{k_{0}}{\lambda_{0}} \sum_{l=1}^{q} \beta_{l}^{2}\right)^{-1} e \quad m_{1}=v_{1}\left[\frac{m_{0}}{v_{0}}+\frac{k_{0} \sum_{l=1}^{q} \beta_{l}\left(\bar{w}_{0 l}-\alpha_{l}\right)}{\lambda_{0} \sum_{l=1}^{q} \beta_{l}^{2}}\right] \text {. }
\end{aligned}
$$

As quantidades $\hat{\alpha}_{l}, \hat{\beta}_{l}$ são os estimadores de máxima verossimilhança associados a l-ésima reta de regressão.

\subsection{Análise de dados citogenéticos}

O dados analisados a seguir foram apresentados no trabalho de mestrado de Madruga (1994) e posteriormente utilizados em Madruga, Ochi-Lohmann, Okazaki and Pereira (1996). A tabela 5.1 apresenta a frequência de células com micronúcleo( $\mathrm{MN})$ em 4 indivíduos sadios expostos a 8 diferentes doses de radiação Gama. A presença de MN indica uma aberração na célula. Foram considerados três grupos distintos de células, caracterizados pelo tipo de linfócitos: mononucleados, binucleados e multinucleados.

Consideramos a transformação

$$
x_{i}=\log \left(d_{i}+1\right), i=1,2, . ., 8,
$$

onde $d_{i}$ representa o i-ésimo valor de dose, previamente fixado. 
Para cada um dos grupos ajustamos um modelo probit, dado por

$$
y_{i j} \mid x_{i} \sim B\left(k_{i j}, p_{i j}\right), \quad \operatorname{com} p_{i j}=\Phi\left(\alpha+\beta x_{i}\right), \quad i=1, . ., 8 ; j=1,2 .
$$

Onde

$y_{i j}$ é a frequência de células com MN, associada ao i-ésimo valor de dose e ao j-ésimo grupo;

$k_{i j}$ é o número de células submetidas a i-ésima dose, associada ao j-ésimo grupo;

$p_{i j}$ é a probabilidade da célula, submetida a i-ésima dose e pertencente ao j-ésimo grupo, apresentar pelo menos um MN.

Tabela 5.1: Frequência de MN para diferentes doses de radiação Gama

\begin{tabular}{|c|c|c|c|c|c|c|}
\hline \multirow{2}{*}{$\begin{array}{r}\text { Dose } \\
d_{i} \\
\end{array}$} & \multicolumn{2}{|c|}{ Mononucleados } & \multicolumn{2}{|c|}{ Binucleados } & \multicolumn{2}{|c|}{ Multinucleados } \\
\hline & $y_{i 1}$ & $k_{i 1}$ & $y_{i 2}$ & $k_{i 2}$ & $y_{i 2}$ & $k_{i 2}$ \\
\hline 0 & 35 & 51272 & 32 & 2373 & 2 & 84 \\
\hline 20 & 109 & 24000 & 51 & 2662 & 7 & 150 \\
\hline 50 & 204 & 26892 & 142 & 1991 & 20 & 183 \\
\hline 100 & 521 & 26437 & 236 & 2047 & 26 & 164 \\
\hline 200 & 1067 & 24549 & 407 & 2611 & 21 & 112 \\
\hline 300 & 821 & 9344 & 708 & 2442 & 56 & 148 \\
\hline 400 & 1003 & 10811 & 777 & 2398 & 50 & 154 \\
\hline 500 & 1130 & 8814 & 741 & 1746 & 30 & 59 \\
\hline
\end{tabular}

Ajustando o modelo pelo método de máxima verossimilhança, obtivemos os resultados gráficos da figura 5.2, que relacionam os valores $x_{i}$ (eixos horizontais) com os $p_{i j}$ (eixos verticais). 
Figura 5.2: Gráficos de ajustes $\left(x_{i} \times p_{i j}\right)$
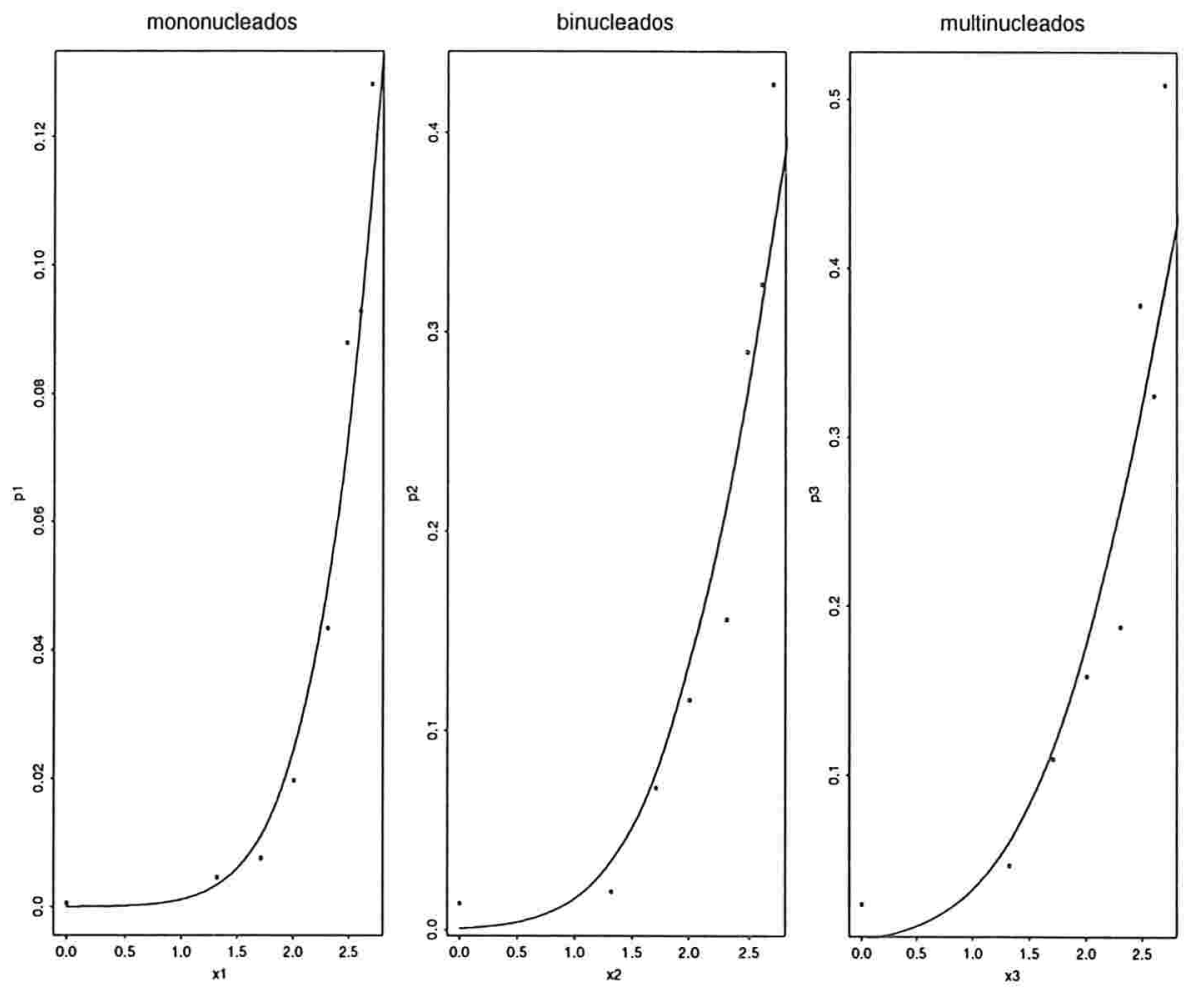

O objetivo do estudo é utilizar a análise citogenética, que observa o número de $\mathrm{MN}$ nas células, para predizer a dose de radiação Gama a qual um indivíduo foi submetido. Para avaliar a qualidade da metodologia proposta neste capítulo, procedemos da seguinte maneira:

(a) para cada tipo de linfócitos, desconsideramos uma das linhas da tabela 5.1 e consideramos as 7 quantidades restantes como um experimento de calibração;

(b) os valores de $y$ e $k$ da linha eliminada fazem o papel de $y_{0}$ e $k_{0}$, respectivamente.

Desta maneira, para cada linha buscamos recuperar o valor de dose, denotado por $d_{0}$. Inicialmente estimamos $x_{0}$ e depois recuperamos $d_{0}$ através da relação $d_{0}=10^{x_{0}}-1$.

Nas tabelas 5.2, 5.3 e 5.4 apresentamos os resultados obtidos com a aproximação normal. 
Tabela 5.2: Resultados assintóticos para $x_{0}$ e $d_{0}$, linfócitos mononucleados

\begin{tabular}{|c|c|c|c|c|c|c|}
\hline$d_{0}$ & $x_{0}$ & $E\left[x_{0}\right]$ & $D P\left[x_{0}\right]$ & $\begin{array}{l}\text { R.C.de } x_{0} \\
\quad(0.90)\end{array}$ & $\hat{d}_{0}$ & $\begin{array}{l}\text { R.C.de } d_{0} \\
\quad(0.90)\end{array}$ \\
\hline 0 & 0 & 1.022 & 0.0464 & $(0.99457,1.0989)$ & 9.527 & $(7.8253,11.5560)$ \\
\hline 20 & 1.322 & 1.443 & 0.0351 & $(1.3845,1.5005)$ & 26.705 & $(23.24076,30.6612)$ \\
\hline 50 & 1.708 & 1.513 & 0.0313 & $(1.4613,1.5646)$ & 31.578 & $(27.9248,35.6935)$ \\
\hline 100 & 2.004 & 1.890 & 0.0205 & $(1.8565,1.9241)$ & 76.679 & $(70.8623,82.9667)$ \\
\hline 200 & 2.303 & 2.228 & 0.0151 & $(2.2029,2.2527)$ & 167.973 & $(158.5669,177.9341)$ \\
\hline 300 & 2.479 & 2.603 & 0.0199 & $(2.5706,2.6364)$ & 400.327 & $(371.0248,431.9364)$ \\
\hline 400 & 2.603 & 2.610 & 0.0187 & $(2.5794,2.6413)$ & 406.716 & $(378.6724,436.8295)^{*}$ \\
\hline 500 & 2.700 & 2.838 & 0.0220 & $(2.8019,2.8745)$ & 687.998 & $(632.7134,748.1057)$ \\
\hline
\end{tabular}

* intervalo que contem o verdadeiro valor $d_{0}$.

Para os grupos binucleados e multinucleados desconsideramos os valores extremos de dose, $d=0$ e $d=500$, devido ao evidente problema que teríamos para recuperar estas quantidades com base nos ajustes apresentados na Figura 5.1.

Tabela 5.3. Resultados assintóticos para $x_{0}$ e $d_{0}$, linfócitos binucleados

\begin{tabular}{|c|c|c|c|c|c|c|}
\hline$d_{0}$ & $x_{0}$ & $E\left[x_{0}\right]$ & $D P\left[x_{0}\right]$ & $\begin{array}{c}\text { R.C.de } x_{0} \\
\quad(0.90)\end{array}$ & $\hat{d}_{0}$ & $\begin{array}{c}\text { R.C.de } d_{0} \\
\quad(0.90)\end{array}$ \\
\hline 20 & 1.322 & 0.912 & 0.0820 & $(0.7763,1.0468)$ & 7.23 & $(4.9750,10.1379)$ \\
\hline 50 & 1.708 & 1.645 & 0.0485 & $(1.5654,1.7254)$ & 43.20 & $(35.7641,52.1344)^{*}$ \\
\hline 100 & 2.004 & 1.899 & 0.0397 & $(1.8337,1.9648)$ & 78.29 & $(67.1798,91.2071)$ \\
\hline 200 & 2.303 & 2.056 & 0.0318 & $(2.0035,2.1085)$ & 112.78 & $(99.8123,127.3768)$ \\
\hline 300 & 2.479 & 2.558 & 0.0306 & $(2.5080,2.6090)$ & 360.82 & $(321.1265,405.4185)^{*}$ \\
\hline 400 & 2.603 & 2.646 & 0.0318 & $(2.5933,2.6983)$ & 441.38 & $(391.0157,498.2022)^{*}$ \\
\hline
\end{tabular}

* intervalos que contêm o verdadeiro valor $d_{0}$. 
Tabela 5.4. Resultados assintóticos para $x_{0}$ e $d_{0}$, linfócitos multinucleados

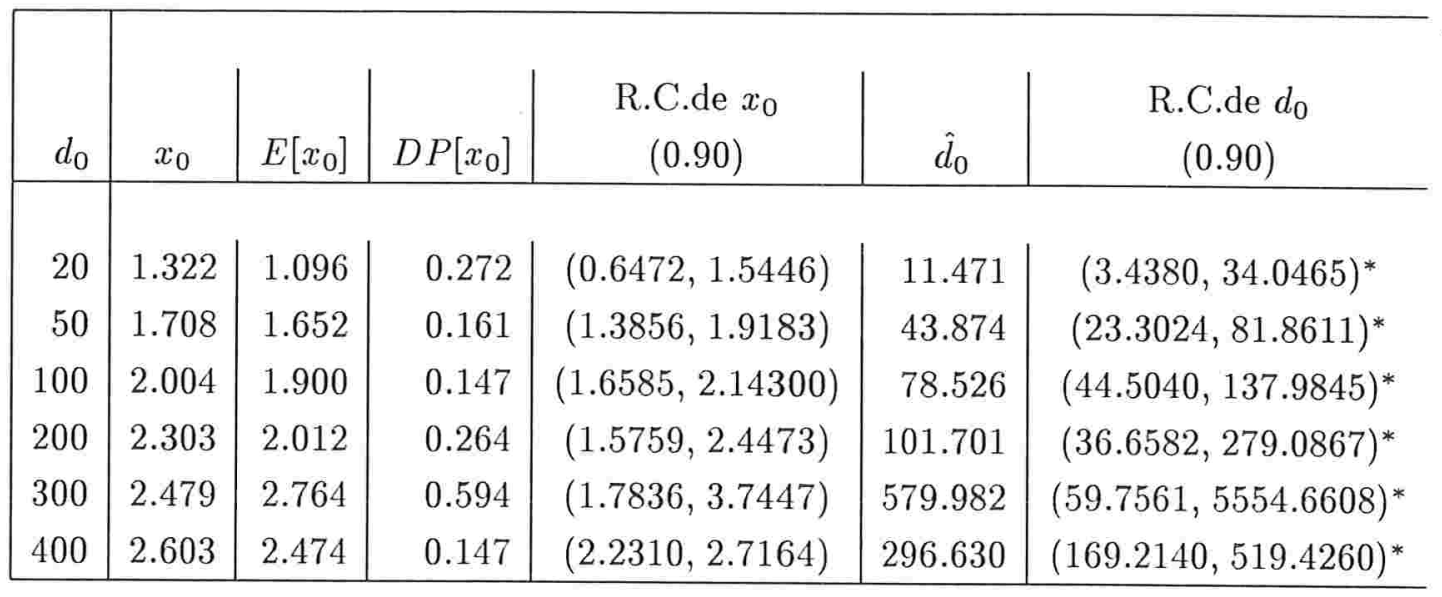

* intervalos que contêm o verdadeiro valor $d_{0}$.

Observe que muitas vezes as regiões de credibilidade não contêm o verdadeiro valor da dose. Esta situação é mais crítica para o grupo dos mononucleados, mesmo sendo este o de melhor ajuste, e fica mais amena no grupo dos multinucleados. No entanto, os intervalos gerados para os multinucleados são pouco precisos. Isto deve-se ao fato do primeiro grupo possuir maiores valores para os $k_{i}^{\prime} s$ e menores valores para o desvio padrão assintótico.

De um modo geral podemos dizer que a aproximação assintótica para normal não parece muito adequada para o problema. Uma das justificativas é que, mesmo que o número total de células examinadas seja muito grande, o númeo de elementos no experimento de calibração é pequeno $n=7$.

Para a obtenção das amostras de Gibbs, elaboramos um programa em S-Plus. A convergência da cadeia foi verificada considerando-se as etapas descritas a seguir.

(i) Consideramos seis pontos distintos como valores iniciais e, após 600 iterações, analisamos graficamente o comportamento das cadeias e das médias ergodicas. Após a análise dos gráficos do apêndice B.1, decidimos descartar as 100 primeiras iterações antes da análise de convergência, por evidenciarem uma grande variabilidade.

(ii) Desconsiderado-se as 100 iterações iniciais, utilizamos os mesmos seis pontos iniciais para gerar seis cadeias de tamanho 1000 (ver gráficos do apêndice B.2). Obtendo-se o seguinte valor para a estatística de Gelman and Rubin (1992) $R=1.0156$, que indica a convergência da cadeia. 
(iii) Para a contrução das amostras, desconsideramos as 1100 iterações iniciais e utilizamos uma única cadeia de tamanho $m=1500$. Os resultados obtidos destas amostras para as estimativas das médias e desvio padrões a posteriori para a dose, bem como a região de $90 \%$ de credibilidade, são apresentados na Tabela 5.5 para o grupo multinucleados. Na figura 5.3 apresentamos os histogramas associados as amostras das doses.

Tabela 5.5. Resultados via algoritmo DA para $d_{0}$, linfócitos multinucleados

\begin{tabular}{r|r|r|r|c}
\hline & \multicolumn{3}{|c}{} \\
$d_{0}$ & \multicolumn{1}{|c|}{$x_{0}$} & $E\left[d_{0}\right]$ & $D P\left[d_{0}\right]$ & $\begin{array}{c}\text { R.C.de } d_{0} \\
(0.90)\end{array}$ \\
\hline & & & \\
20 & 1.322 & 11.08 & 8.14 & $(1.2727,26.5576)^{*}$ \\
50 & 1.708 & 45.98 & 17.52 & $(22.9036,78.0681)^{*}$ \\
100 & 2.004 & 82.89 & 27.65 & $(44.5288,133.3661)^{*}$ \\
200 & 2.303 & 106.75 & 40.33 & $(53.3385,185.5021)$ \\
300 & 2.479 & 659.38 & 298.91 & $(328.77,1199.0292)$ \\
400 & 2.603 & 345.19 & 137.58 & $(191.5866,595.3915)^{*}$ \\
\hline
\end{tabular}

* intervalos que contêm o verdadeiro valor $d_{0}$.

As estimativas pontuais obtidas são, em geral, melhores do que as obtidas pelo estimador de máxima verossimilhaça (média assintótica). Além disso, as regiões de credibilidades são mais precisas, isto é, tem menor amplitude. Encontramos problemas em duas regiões de credibilidade $(\mathrm{d}=200 \mathrm{e} \mathrm{d}=300)$ que não cobrem o verdadeiro valor de dose. Se observarmos novamente a Figura 5.2, verificamos que estes valores de doses estão associados aos pontos no gráfico que estão mais afastados da curva ajustada.

A figura 5.4 mostra o comportamento estimado das densidades marginais a posteriori de $x_{0}, \beta$ e $\alpha$, através dos histogramas gerados das amostras de Gibbs e das densidades estimadas a partir das densidades condicionais, quando $d_{0}=50$ e $x_{0}=1.71$. ( ver apêndice B.3 para outros valores de $d_{0}$ ) 
Figura 5.3: Histogramas para $d_{0}(\mathrm{~m}=1500)$
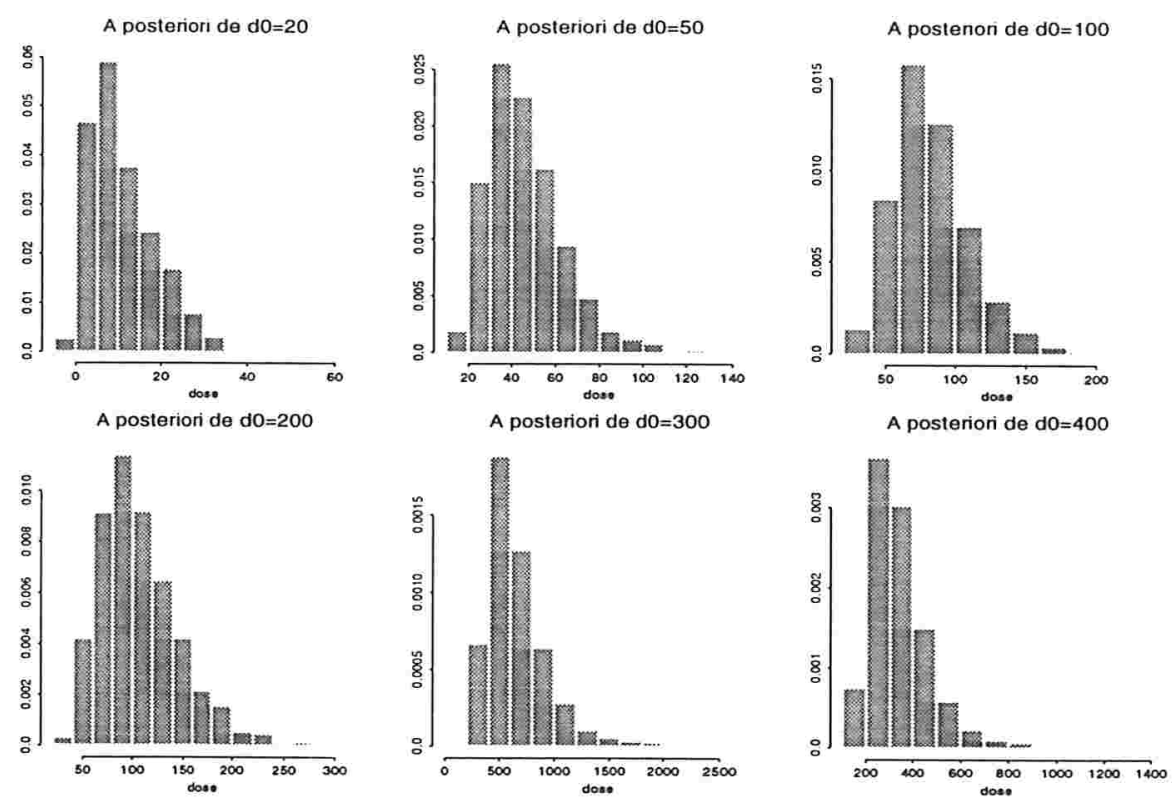

Figura 5.4: Densidades estimadas para $d_{0}=50$
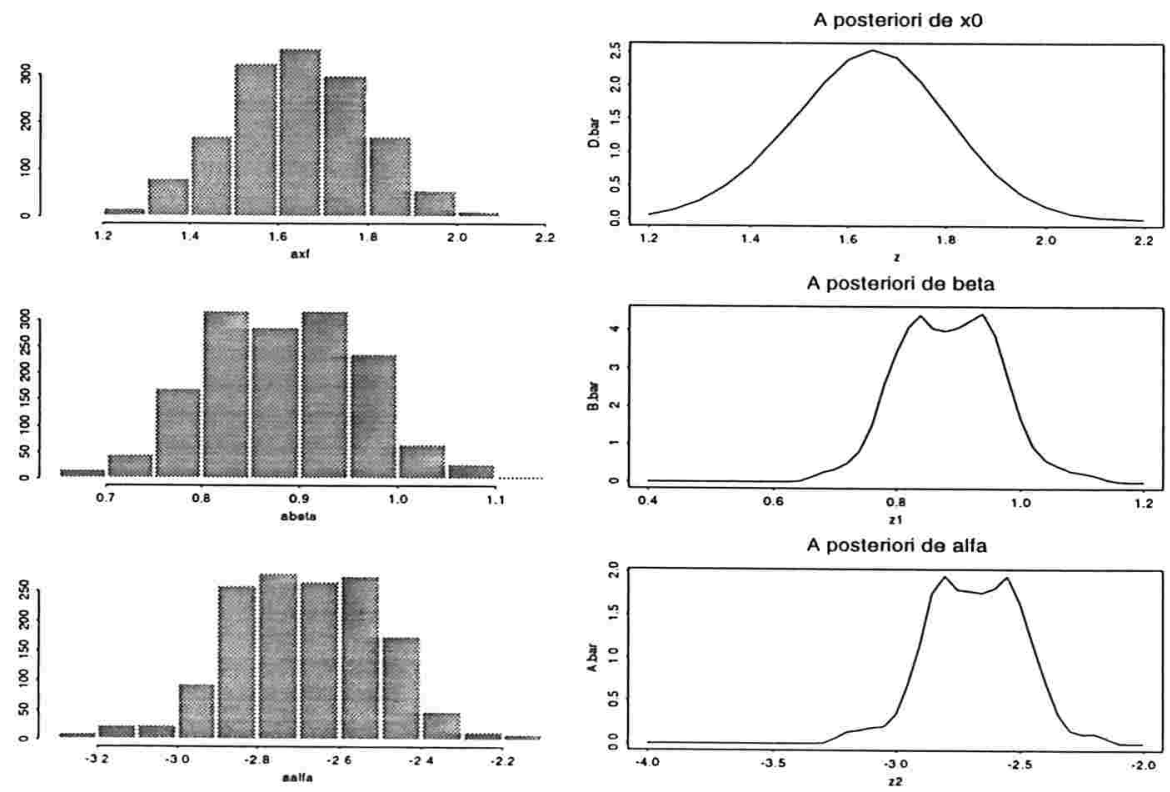


\section{Capítulo 6}

\section{Considerações finais}

\subsection{Conclusões}

Este trabalho estende alguns resultados que tem aparecido na literatura em várias direções:

1. O tradicional modelo de calibração linear foi analizado sob a suposição de que os erros têm distribuição elíptica multivariada com matriz de dispersão diagonal. Neste caso, os erros são não correlacionados mas não necessariamente independentes, salvo no caso normal. Esta classe inclui modelos como a família t-multivariada, misturas no parâmetro de escala de normais multivariadas, Pearson tipo II, etc. A análise bayesiana do problema utilizando-se uma distribuição a priori de referência para o parâmetro de dispersão, mostrou-se invariante dentro desta classe das distribuições elípticas. A relevância deste resultado está no fato de que os vários resultados para o problema de calibração sob normalidade permanecem válidos para a classe completa das distribuições elípticas multivariadas com matriz de dispersão diagonal. Mostrando, por exemplo, que o estimador inverso é robusto diante do afastamento da normalidade. Estes resultados foram estendidos para o problema de calibração multivariado, verificando-se o mesmo tipo de robustez.

2. Ainda sob a suposição do modelo elíptico dependente, resultados foram obtidos para o problema de calibração considerando-se uma distribuição a priori conjugada para o modelo de regressão. Como subproduto mostrou-se que o estimador de bayes para os coeficientes de regressão coincide com o obtido na análise conjugada do modelo de regressão normal. Portanto, a característica do estimador de Bayes sob perda quadrática (média a posteriori) de ser uma combinação linear convexa das 
observações não é, como sugere Goel and DeGroot (1980), uma particularidade dos modelos normais; e sim uma característica de toda a classe das elípticas. O ponto central desta extenção está em substituírmos a hipótese de independência dos erros pela de não correlação.

3. Sob a suposição de erros elípticos independentes, os resultados de robustez derivados para o modelo dependente não são válidos. Neste modelo observa-se dificuldades em obter-se resultados analíticos tratáveis. Porém, para modelos elípticos representáveis, isto é, misturas de normais(que inclui os modelos normais contaminados, t-student, cauchy, etc) foi possível obter uma forma geral para a distribuição a posteriori associada ao problema de calibração. Mais ainda, a suposição de representabilidade possibilitou a obtenção de formas conhecidas para as distribuições condicionais a posteriori, permitindo estabelecer um método geral para o uso do amostrador de Gibbs. A importância deste estudo está no fato de que muitas das distribuições dentro desta classe são menos sensíveis a observações extremas que o modelo normal. Deste modo, as estimativas obtidas sob estes modelos são mais robustas, no sentido de não serem tão afetadas por observações extremas. Estes resultados, também, foram estendidos para o problema de calibração multivariado. Finalmente na aplicação que considera o modelo com erros t-Student, verificamos que a obtenção da distribuição a posteriori através do algoritmo proposto, com o auxílio do pacote BUGS, é muito rápida (menos de 10 segundos) e que, partindo deste modelo mais geral, temos o caso normal como um caso limite. Mesmo no caso normal há vantagens na técnica sugerida, pois não há necessidade de ficarmos restritos a distribuição a priori proposta por Hoadley (1970), para obtenção da distribuição e das estimativas a posteriori.

4. Enquanto no caso do modelo elíptico dependente o uso, mesmo que questionável, de uma distribuição a priori de referência foi positivo no sentido de pemitir a obtenção de resultados que facilitam a análise. No caso do modelo independente, o uso destas distribuições acarreta dificuldades extras.Em primeiro lugar, tivemos que estabelecer condições que garantissem a existência da distribuição a posteriori. As condições estabelecidas nem sempre são fáceis de verificar. A não existência de uma distribuição a posteriori própria não recai somente num problema teórico de incoerência, pelo fato de não estarmos trabalhando com uma distribuição de probabilidades; mas também é um grande problema na aplicação do amostrador de Gibbs. Pois, caso não 
exista uma distribuição de probabilidade a posteriori (própria) a convergência do algoritmo não estará garantida. Um estudo similar, que discute a existência da distribuição a posteriori, foi apresentado por Geweke (1993) para modelos de regressão com erros $t$ independentes. Porém, de acordo com a nossa análise do trabalho, suas provas não estão certas.

4.Na análise de dados categorizados as dificuldades no tratamento analítico são ainda maiores, não sendo possível, em geral, estabelecer resultados analíticos explícitos. Nesta direção a idéia fundamental foi considerar o uso de variáveis latentes que possibilitou a implementação do algoritmo DA, tanto para o modelo binomial quanto para o multinomial. Os resultados obtidos são gerais, pois permitem trabalhar com funções de enlace dentro da classe das distribuições elípticas (incluindo a normal e a logística). Uma aproximação assintótica para a distribuição a posteriori, no modelo binomial, foi apresentada com o objetivo de comparar os resultados. Finalmente, na aplicação à dados citogenéticos podemos verificar que os resultados via DA são aparentemente melhores que os resultados via aproximação assintótica. Já que as estimativas pontuais estiveram mais próximas do verdadeiro valor a ser estimado e as regiões de credibilidades são mais precisas. Além disso, as regiões de credibilidades, construídas pelo método DA, para a variável original (dose de radiação Gama) baseam-se na verdadeira distribuição a posteriori de $d_{0}$ e não apenas na transformação dos limites do intervalo como é o procedimento fornecido pela aproximação normal. As vantagens da aproximação assintótica são, claramente, o tempo e a simplicidade. Para obtermos as estimativas da média e da variância de $x_{0}$, necessitamos apenas de um pacote estatístico e de alguns segundos para execução. Já os resultados via DA dependeram da formulação de um programa que demorou em média 6 horas(no grupo dos multinucleados), para a obtenção da amostra e das estimativas. Além disso, este tempo cresce com o crescimento dos valores dos $k_{i}^{\prime} s$ (tamanho das amostras).

\subsection{Perspectivas futuras}

Com relação ao trabalho futuro são várias a linhas de pesquisa que se abrem a partir deste estudo: 
1. O estudo de aproximações assintóticos para o problema de calibração considerando erros elípticos independentes.

2. O estudo de soluções considerando erros elípticos independentes não representáveis.

3. O estudo de condições para a validade da aproximação assintótica nos modelos discretos. Esta situação tem sido estudada para modelos lineares generalizados apenas no caso clássico. A aproximação assintótica da distribuição a posteriori tem dificuldades extras como indica o trabalho de Chen (1985).

4. A complementação da análise dos dados citogenéticos, considerando como resposta uma multinomial, onde cada elemento do vetor resposta representa o número de MN por células. Para isso, se faz necessário a implementação do algoritmo para ao modelo multinomial e a busca de uma programa computacional mais eficiente, no sentido de redução do tempo.

5. O estudo da influência das observações nas soluções do problema de calibração utilizando-se medidas de divergência. 


\section{Apêndice A}

\section{O Amostrador de Gibbs}

\section{A.1 Introdução}

O amostrador de Gibbs é um algoritmo iterativo utilizado para gerar amostras de uma determinada distribuição de probabilidades $F(\boldsymbol{x}), \quad \boldsymbol{x} \in \mathbb{R}$, construído a partir de propriedades de Cadeias de Markov. Nos últimos anos vários algoritmos foram propostos com este objetivo e ficaram conhecidos como técnicas MCCM (Monte Carlo baseado em Cadeias de Markov), ver por exemplo, Smith and Roberts (1993), Besag and Green (1993) e Gamerman (1996). O procedimento básico destas técnicas está na construção de uma Cadeia de Markov cuja medida estacionária é a distribuição de interesse $F$ e esta distribuição é estimada a partir de uma amostra, similarmente aos métodos de Monte Carlo. No início dos anos noventa surgiu uma vasta literatura nesta área em revistas estatísticas, por exemplo, Smith and Gelfand (1990), Gelfand, Hills, Racine-Poon and Smith (1990) e Gelman and Rubin (1992). A partir daí os estatísticos, especialmente bayesianos, iniciaram a utilizar metódos MCCM nas mais diversas áreas de aplicações. A grande vantagem destes métodos é sua relativa facilidade de implementação computacional e a grande quantidade de problemas que eles podem resolver.

Apesar do interesse dos estatísticos pelas técnicas MCCM ter ocorrido no final dos anos oitenta e início dos anos noventa, o amostrador de Gibbs já havia sido utilizado por Geman and Geman (1984) para resolver problemas de reconstrução de imagens. Foi deste trabalho que surgiu a denominação Gibbs sampler, pois no problema de reconstrução de imagens trabalhado por Geman and Geman (1984) a distribuição considerada para o modelo é uma distribuição de Gibbs, muito utilizada em mecânica estatística. O amostrador de Gibbs é 
baseado no algoritmo de Metropolis-Hasting (Metropolis, Rosenbluth, Rosenbluth, Teller and Teller 1953), (Hasting 1970). A grande importância de.Geman and Geman (1984) foi introduzir a conecção desta técnica com o problema de inferência bayesiana de obtenção de uma distribuição a posteriori. Em problemas multiparamétricos não é difícil obter-se o núcleo da distribuição a posteriori conjunta de um parâmetro $\theta=\left(\theta_{1}, \theta_{2}, \ldots, \theta_{q}\right)$. No entanto, o mesmo não se pode dizer a respeito das distribuições marginais, associadas a cada $\theta_{i}$. Suponha que nosso interesse seja obter a distribuição associada ao parâmetro $\theta_{1}$, então o algoritmo é construido da seguinte maneira:

(i) A partir da distribuição conjunta $F(\theta)$ obtem-se todas as distribuições condicionais $F\left(\theta_{i} \mid \theta_{-i}\right)$, onde $\theta_{-i}=\left(\theta_{1}, \ldots, \theta_{i-1}, \theta_{i+1}, \ldots, \theta_{q}\right)$ é o vetor de parâmetros quando retirado $\theta_{i}$. Estas distribuições condicionais devem ser distribuições conhecidas, das quais sejam facilmente geradas amostras.

(ii) Fixam-se valores iniciais $\theta_{1}^{(0)}, \ldots, \theta_{q}^{(0)}$ para os parâmetros $\theta_{1}, \ldots, \theta_{q}$, respectivamente.

(iii) Gera-se um novo conjunto de valores $\theta_{1}^{(k+1)}, \theta_{2}^{(k+1)}, . ., \theta_{q}^{(k+1)}$ a partir das seguintes distribuições $F\left(\theta_{1} \mid \theta_{2}^{(k)}, \ldots, \theta_{q}^{(k)}\right), F\left(\theta_{2} \mid \theta_{1}^{(k+1)}, \theta_{3}^{(k)}, \ldots, \theta_{q}^{(k)}\right), \ldots, F\left(\theta_{q} \mid \theta_{1}^{(k+1)}, \ldots, \theta_{q-1}^{(k+1)}\right)$, respectivamente, para $k=0,1, . ., t$.

(iv) Os valores $\theta_{1}^{(t)}, \theta_{2}^{(t)}, \ldots, \theta_{q}^{(t)}$ podem ser considerados como gerados da distribuição conjunta $F\left(\theta_{1}, \ldots, \theta_{q}\right)$, para $t$ suficientemente grande. Em particular, $\theta_{1}^{(t)}$ é um valor gerado da distribuição $F\left(\theta_{1}\right)$. Repetindo-se este procedimento $m$ vezes obtemos uma amostra de tamanho $m$, destas distribuições.

Com a amostra gerada pelo procedimento descrito acima, desenha-se o histograma e obtêm-se estatísticas descritivas como média, desvio padrão e quantis, com o objetivo de aprender sobre a distribuição de interesse $F\left(\theta_{1}\right)$. No caso contínuo, onde o interesse é a densidade associada ao parâmetro $\theta_{1}, f\left(\theta_{1}\right)$, uma estimativa natural para esta densidade é o alisamento deste histograma. Entretanto, como justificado por Smith and Gelfand (1990), pelo teorema de Rao-Blackwell é possível obter um estimador com menor erro quadrático médio utilizando-se as densidades condicionais $f\left(\theta_{i} \mid \theta_{-i}\right)$ do seguinte modo

$$
\hat{f}\left(\theta_{1}\right)=\frac{1}{m} \sum_{t=1}^{m} f\left(\theta_{1} \mid \theta_{2}^{(t)}, \ldots, \theta_{q}^{(t)}\right) .
$$

A justificativa para o procedimento apresentado de (i) a (iv) provêm do fato de que os valores $\theta^{(1)}, \theta^{(2)}, \ldots, \theta^{(t)}, \ldots$ são uma realização de uma cadeia de Markov com núcleo de transiçâo dado por 


$$
K\left(\theta^{(t)}, \theta^{(t+1)}\right)=\prod_{i=1}^{q} F\left(\theta_{i}^{(t+1)} \mid \theta_{-i}^{(t)}\right)
$$

e que, sob algumas condições de regularidades, tem como distribuição estacionária $F(\theta)$. As condições exigidas para convergência da cadeia, isto é, para a existência de uma medida. estacionária são de que ela seja irredutível e aperiódica, ver Tierney (1994). Roberts and Smith (1994) obtiveram condições gerais para os casos onde a distribuição de interesse é discreta ou quando existe a uma função densidade $\mathrm{em}^{p}$ (absolutamente contínua). Eles provaram que :

(a) Quando $F$ é uma distribuição de probabilidades discreta, então a $K\left(\theta^{(t)}, \theta^{(t+1)}\right)$ está bem definido e a cadeia é aperiódica. Portanto, verificar a irredutibilidade da cadeia é suficiente para a convergência.

(b) Quando $F$ é uma função de distribuição absolutamente contínua e a função de densidade $f$ é inferiormente semi-contínua em zero, então $K\left(\theta^{(t)}, \theta^{(t+1)}\right)$ está bem definido e a cadeia é aperiódica. Além disso, se o espaço paramétrico $\Theta$ é conectado e se cada densidade marginal $f\left(\boldsymbol{\theta}_{-i}\right)$ é localmente limitada, então a cadeia é irredutível e portanto converge.

\section{A.2 Critérios de convergência}

Um dos problemas mais sérios na utilização do amostrador de Gibbs é determinar o número de iterações, a partir do qual, podemos dizer que a cadeia esta em equilíbrio. Diversos métodos foram propostos com este objetivo. Alguns são bastante intuitivos e informais e baseam-se na análise gráfica da trajetória. Outros são mais sofisticados e por vezes pouco operacionais. Recentemente Cowles and Carlin (1996) fizeram uma revisão destes métodos.

Neste trabalho, além da análise gráfica do comportamento da cadeia e das médias ergódicas, consideramos apenas dois citérios numéricos: o método dado por Geweke (1992), baseado em séries temporais; e o de Gelman and Rubin (1992), baseado em múltiplas cadeias.

O método de Gelman and Rubin (1992) consiste em considerar $k$ pontos iniciais distintos e analisar o desenvolvimento das $k$ cadeias. Se após um número $n$ de iterações a variabilidade entre as diversas cadeias estiver próxima da variabilidade interna de uma 
cadeia, então podemos dizer que o equilíbrio foi atingido. Este método segue as idéias de análise de variância, comparando a variabilidade entre as cadeias, dada por

$$
E=\frac{1}{k-1} \sum_{j=1}^{k}\left(\bar{\theta}_{j}-\overline{\bar{\theta}}\right)^{2},
$$

com a variabilidade dentro das cadeias, medida por

$$
D=\frac{1}{k(n-1)} \sum_{j=1}^{k} \sum_{t=1}^{n}\left(\theta_{j}^{(t)}-\bar{\theta}_{j}\right)^{2} .
$$

Onde $\bar{\theta}_{j}$ é a média da j-ésima cadeia, $\overline{\bar{\theta}}$ é a média geral e $\theta_{j}^{(t)}$ é a observação na t-ésima iteração da j-ésima cadeia.

A medida proposta é dada por

$$
R=\left(\frac{n-1}{n}+\frac{E}{D}\right)^{\frac{1}{2}}
$$

Valores de $R$ próximos de 1 indicam convergência, observe que $R$ é sempre maior que 1 . Gelman (1995) sugerem um valor para aceitação da convergência igual a 1.2, isto é, se $R \leq 1.2$ a processo está em equilíbrio.

Críticas a este critério estão relacionadas com a suposição de normalidade que está implícita na construção da análise de variância.

O critério proposto por Geweke (1992) utiliza somente uma cadeia e está baseado em técnicas usuais de séries temporais, considerando que a trajetória $\theta^{(1)}, \theta^{(2)}, \ldots, \theta^{(t)}$ define uma série temporal. O procedimento consiste em considerar duas séries menores. A primeira formada pelos primeiros $n_{I}$ valores da trajetória observada, e a segunda pelos últimos $n_{F}$ valores. Então, considerar a estatística

$$
z=\frac{m e_{I}-m e_{F}}{\left(\hat{S}_{I}(0) / n_{I}+\hat{S}_{F}(0) / n_{F}\right)^{1 / 2}}
$$

para testar a hipótese de que as médias das séries são iguais, onde $m e_{I}$ e $m e_{F}$ são as médias amostrais das séries inicial e final, respectivamente e $\hat{S}_{I}(0)$ e $\hat{S}_{F}(0)$ são as funções densidades espectrais estimadas no ponto zero, que atuam como estimativas da variância do estimador da média. Valores grandes de $z$ indicam a não convergência da cadeia.

A estatística $z$ tem uma distribuição assintótica normal padrão quando $n_{I} / n$ e $n_{F} / n$ são fixos e $n \rightarrow \infty$. Geweke (1992) sugere utilizar $n_{I}=0.1 n$ e $n_{F}=0.5 n$. 


\section{A.3 Comparação com a algoritmo DA}

Na classe dos algoritmos MCCM encontra-se o algoritmo dado por Tanner and Wong (1987) e conhecido como Data Augmentation, o qual nos referimos apenas como DA. Smith and Gelfand (1990) fizeram algumas comparações entre este algoritmo e o amostrador de Gibbs. Tanner (1991) apresenta o amostrador de Gibbs como uma extensão multivariada do seu algoritmo. Vejamos a seguir as idéias desenvolvidas por Tanner (1991) para relacionar os algoritmos. Vamos considerar a situação uniparamétrica onde $\theta$ é o parâmetro de interesse e $\boldsymbol{y}$ são os dados. Além disso, consideramos uma quantidade aleatória $\boldsymbol{z}$ introduzida artificialmente no problema (variável latente). Como antes, o objetivo é obter a distribuição a posteriori para $\theta$. O algoritmo DA é construído com base nas seguintes relações

$$
\begin{gathered}
f(\theta \mid \boldsymbol{y})=\int f(\theta \mid \boldsymbol{y}, \boldsymbol{z}) f(\boldsymbol{z} \mid \boldsymbol{y}) d \boldsymbol{z} \mathrm{e} \\
f(\boldsymbol{z} \mid \boldsymbol{y})=\int f(\boldsymbol{z} \mid \boldsymbol{y}, \theta) f(\theta \mid \boldsymbol{y}) d \theta
\end{gathered}
$$

Denotamos por $f_{i}(\boldsymbol{z} \mid \boldsymbol{y})$ e $f_{i}(\theta \mid \boldsymbol{y})$ as densidades preditiva e a posteriori, respectivamente, no $i$-ésimo estágio de atualização. Estas são atualizadas segundo o esquema abaixo:

(a) Gera-se uma amostra de tamanho $m, \boldsymbol{z}_{1}^{(i)}, \boldsymbol{z}_{2}^{(i)}, \ldots, \boldsymbol{z}_{m}^{(i)}$, de $f_{i}(\boldsymbol{z} \mid \boldsymbol{y})$ repetindo-se $m$ vezes os seguintes passos

(a1) gera-se $\theta^{(i)}$ de $f_{i}(\theta \mid \boldsymbol{y})$;

(a2) gera-se $\boldsymbol{z}^{(i)}$ de $f\left(\boldsymbol{z} \mid \boldsymbol{y}, \theta^{(i)}\right)$.

(b) Atualiza-se o valor da distribuição a posteriori por

$$
f_{i+1}(\theta \mid \boldsymbol{y})=\frac{1}{m} \sum_{j=1}^{m} f\left(\theta \mid \boldsymbol{z}_{j}, \boldsymbol{y}\right)
$$

As etapas descrita em (a) são conhecidas como etapas de imputação e (b) como etapa da posteriori, ver Tanner (1991). Quando $m=1$ o procedimento do DA resume-se a. 
(i) gera-se $\theta^{(i)}$ de $f_{i}(\theta \mid \boldsymbol{y})=f\left(\theta \mid \boldsymbol{z}^{(i-1)}, \boldsymbol{y}\right)$;

(ii) gera-se $\boldsymbol{z}^{(i)}$ de $f\left(\boldsymbol{z} \mid \boldsymbol{y}, \theta^{(i)}\right)$.

Se pensarmos em $z$ como mais um parâmetro do modelo, as etapas (i) e (ii) são exatamente o procedimento do amostrador de Gibbs. Portanto, o DA com $\mathrm{m}=1$ é equivalente ao amostrador de Gibbs.

Este procedimento muitas vezes é referido na literatura, por exemplo, Albert and Chib (1993), como um procedimento que mescla os dois algoritmos, onde Utiliza-se a idéia de Tanner and Wong (1987) de introduzir variáveis latentes ao problema e o amostrador de Gibbs para gerar as amostras. 


\section{Apêndice B}

\section{Gráficos}

Neste apêndice apresentamos alguns gráficos obtidos na análise dos dados citogenéticos via algoritmo DA descrita na seção 5.4.

B.1. Gráficos da pré-análise da convergência. Para cada uma das seis cadeias, com pontos iniciais distintos, foram considerados dois gráficos: um que descreve a trajetória da cadeia e outro das médias ergódicas. Para cada cadeia consideramos $t=600$ iterações e $x_{0}=1.71$. Os gráficos apresentados consideram o parâmetro $x_{0}$, que esta sendo denotado por xf.

B.2. Gráficos da análise de convergência. Para cada uma das seis cadeias foram considerados dois gráficos: um que descreve a trajetória da cadeia e outro das médias ergódicas. Para cada cadeia descartamos as 100 iterações iniciais e consideramos as $t=1000$ iterações seguintes, com $d_{0}=50$. Os gráficos apresentados consideram diretamente o parâmetro de interesse $d_{0}$.

B.3. Gráficos das densidades estimadas para $x_{0}, \beta$ e $\alpha$. Apresentamos os histogramas e as densidades estimadas considerando-se as distribuições condicionais, baseados nas amostras de tamanho $m=1500$. 
B.1. Gráficos da pré-análise da convergência
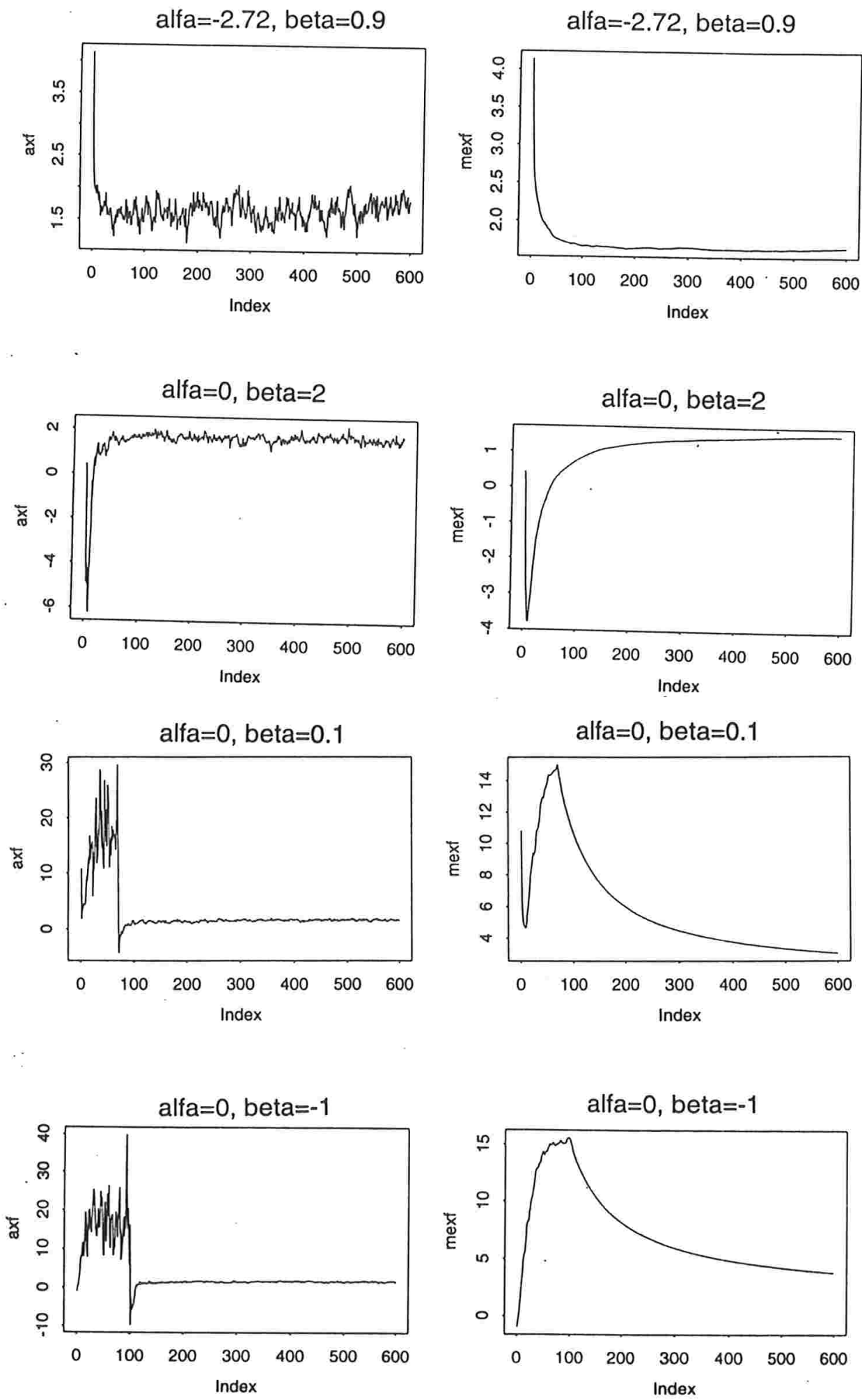
B.1. Gráficos da pré-análise da convergência
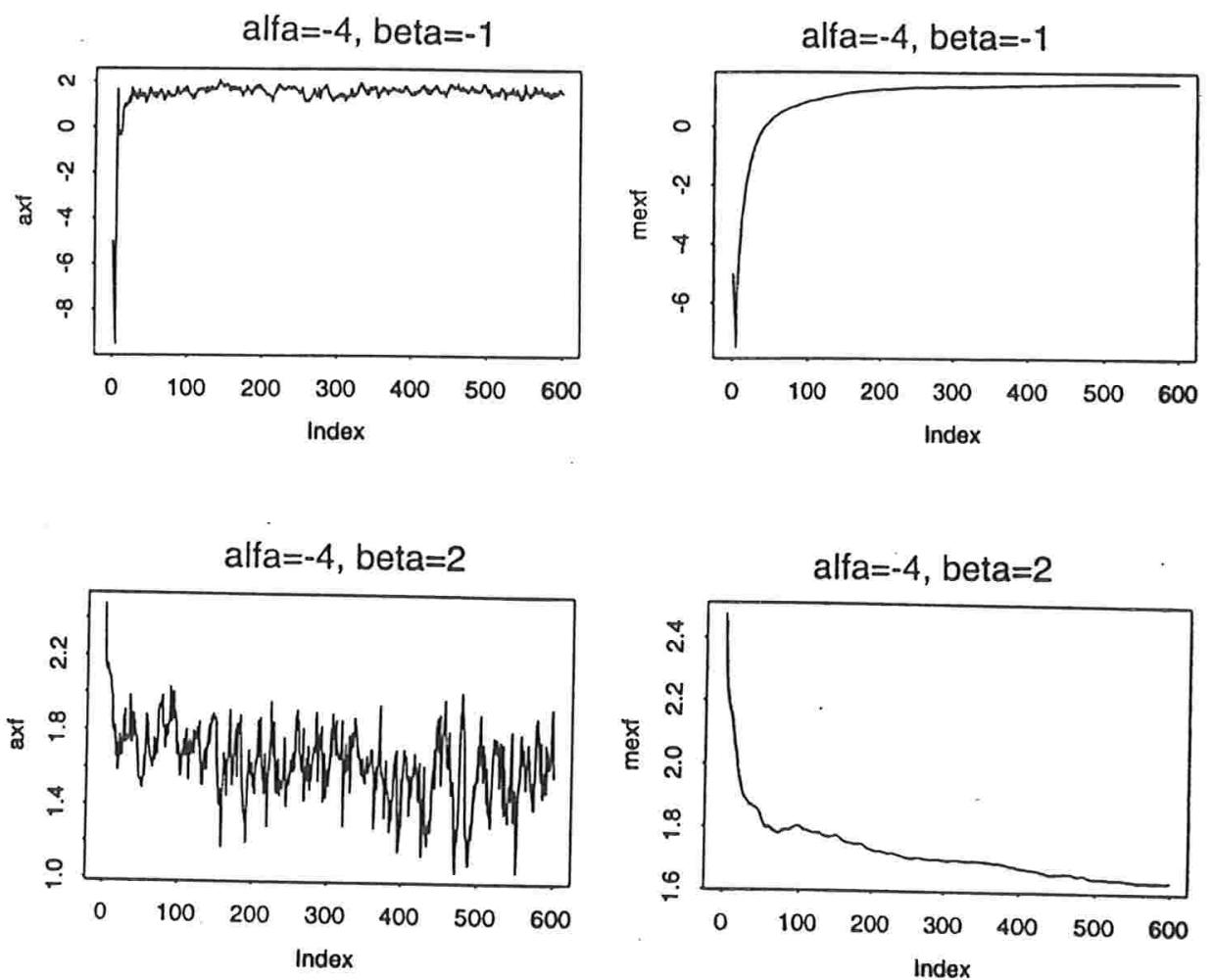
B.2. Gráficos da análise de convergência
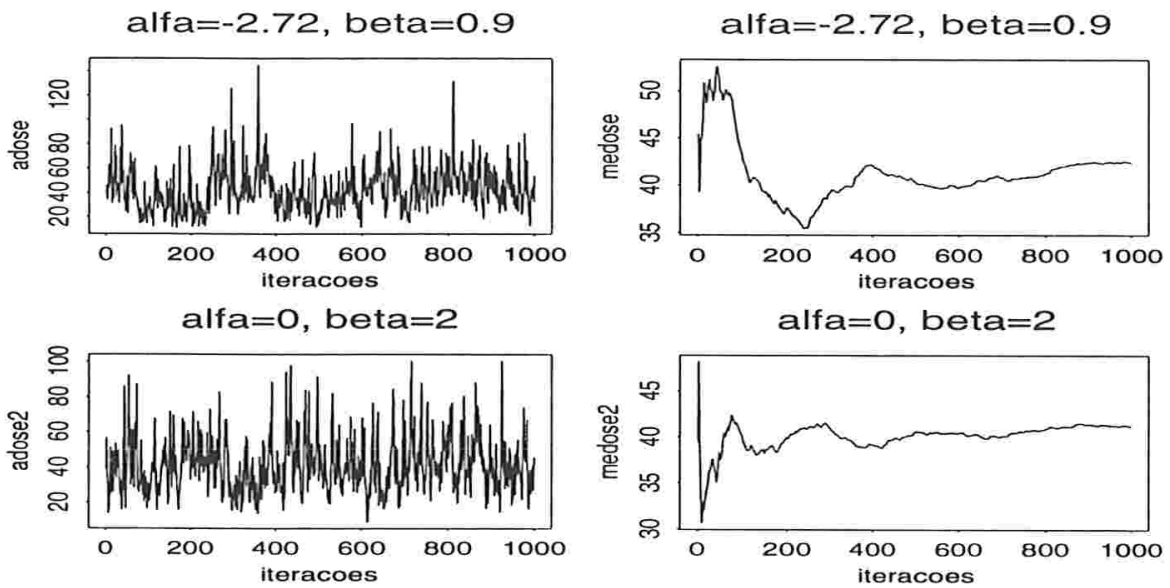

alfa $=0$, beta $=0.1$

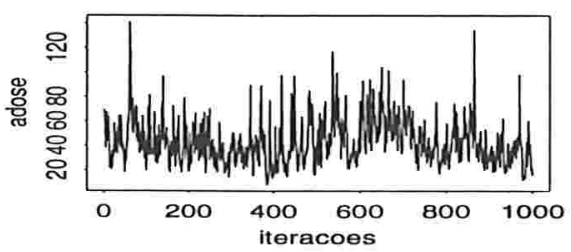

alfa $=0$, beta $=0.1$

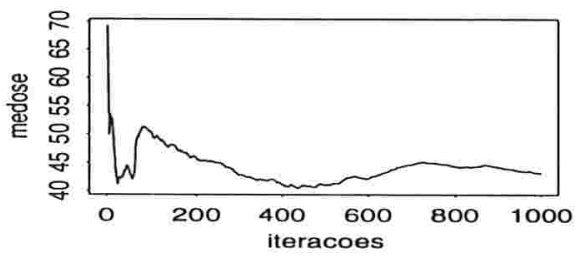

alfa $=0$, beta $=-1$
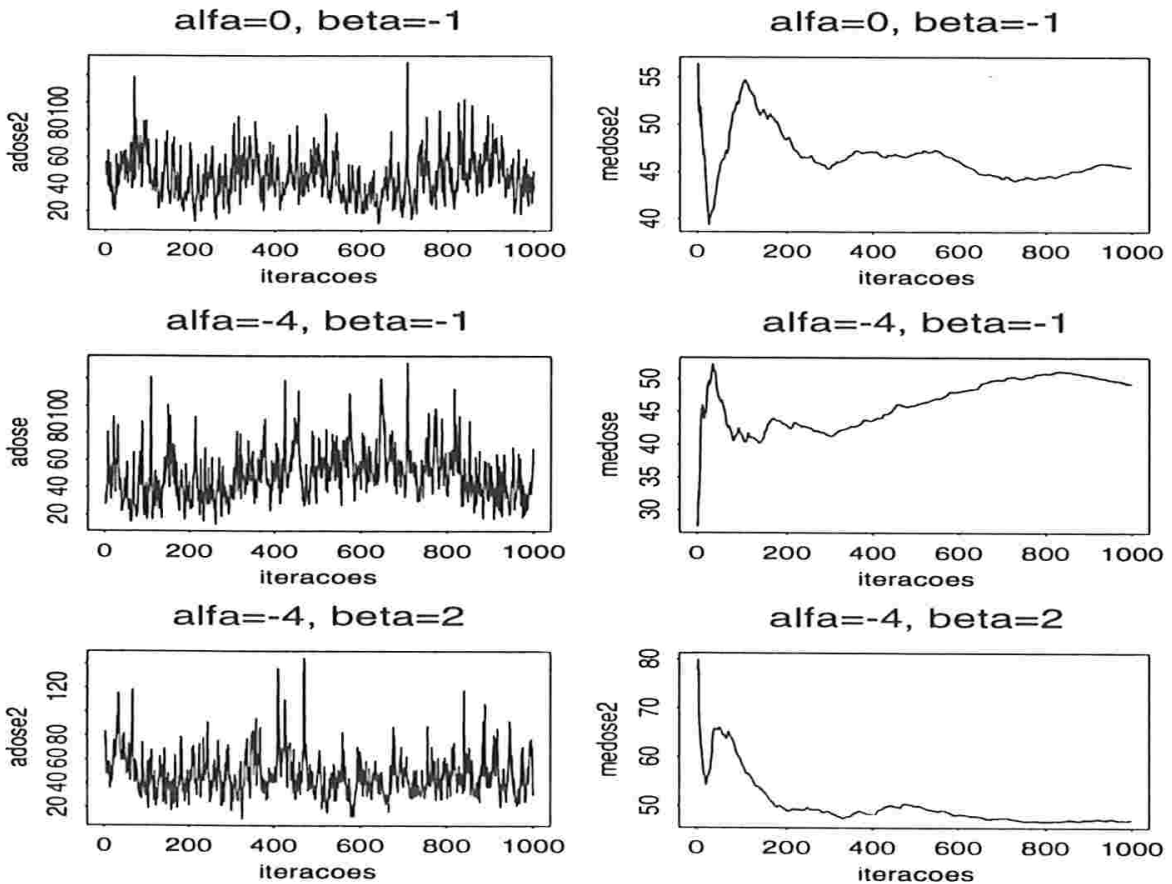
B.3. Gráficos das densidades estimadas para $x_{0}, \beta$ e $\alpha$

Figura B.3.1: Densidades estimadas para $d_{0}=20$ e $x_{0}=1.32$
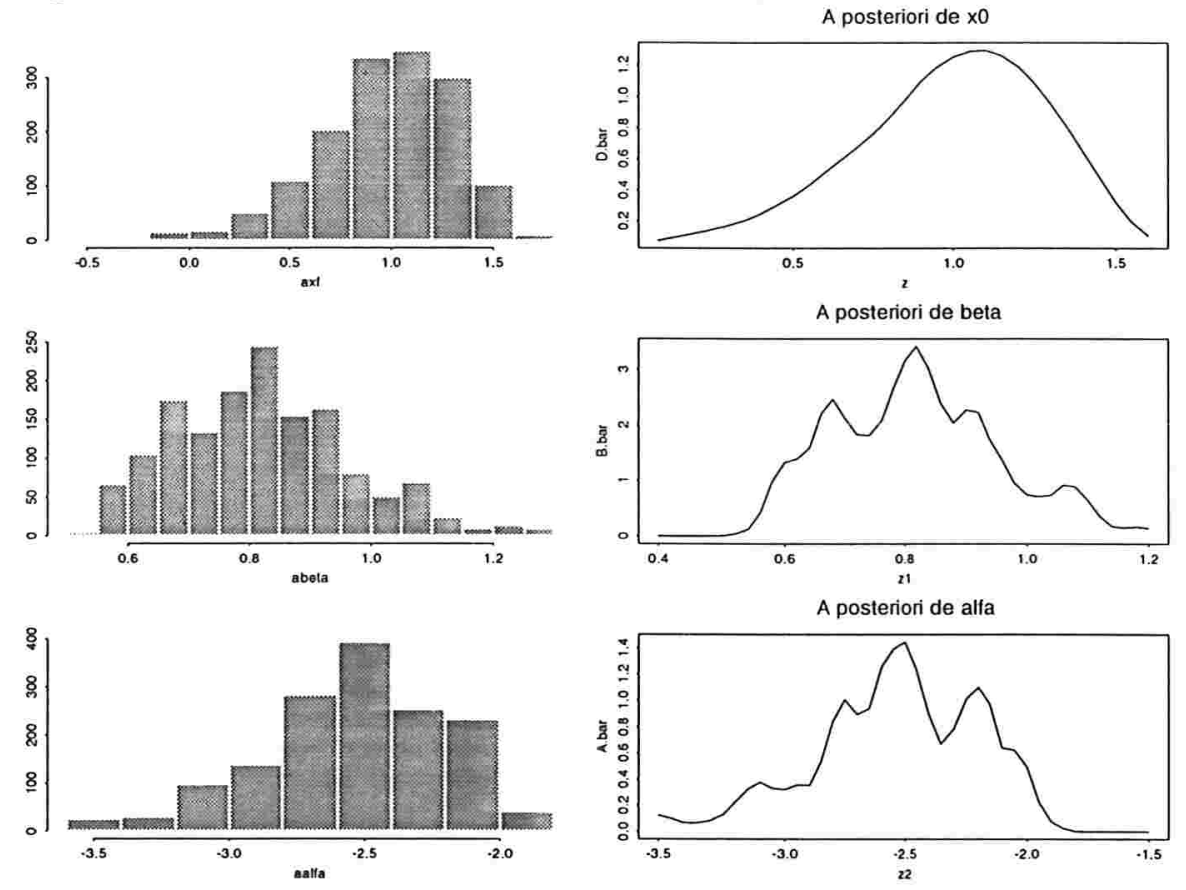

Figura B.3.2: Densidades estimadas para $d_{0}=50$ e $x_{0}=1.71$
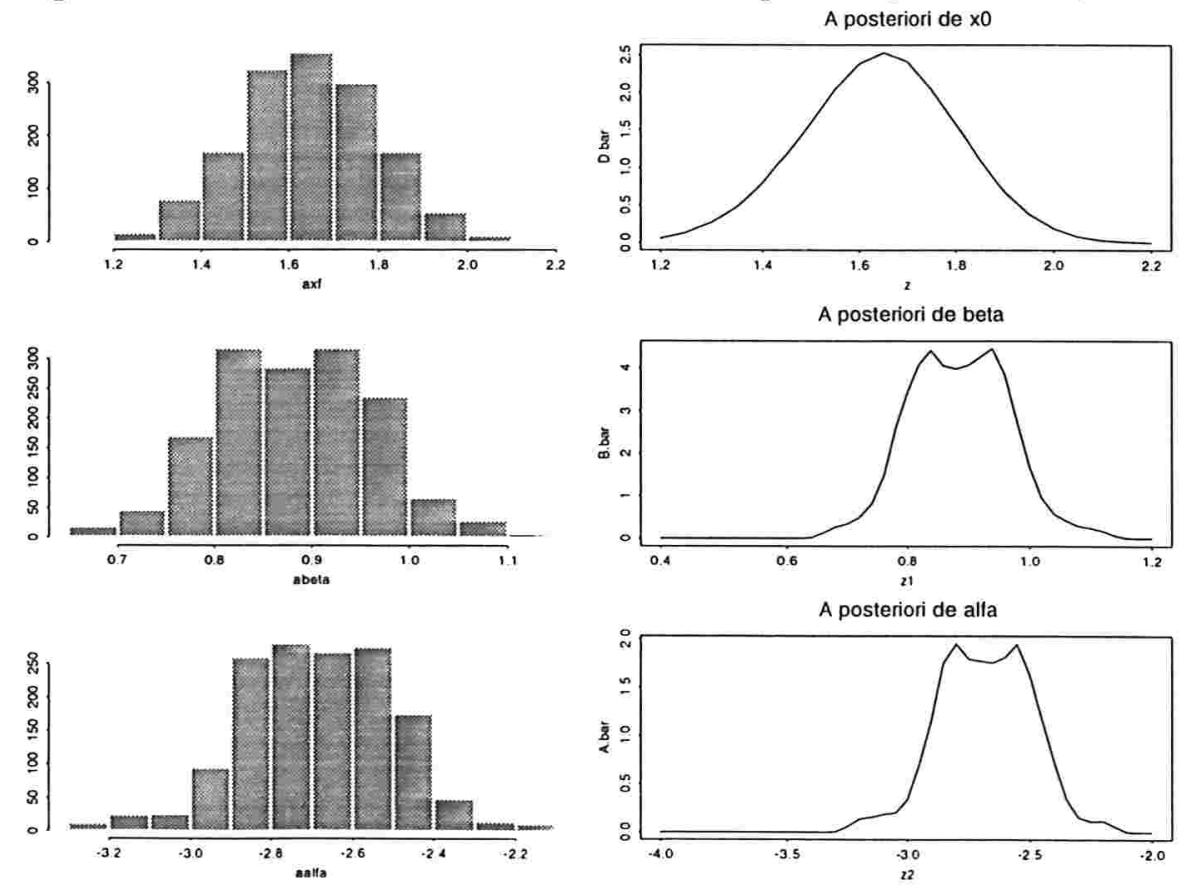
Figura B.3.3: Densidades estimadas para $d_{0}=100$ e $x_{0}=2.00$
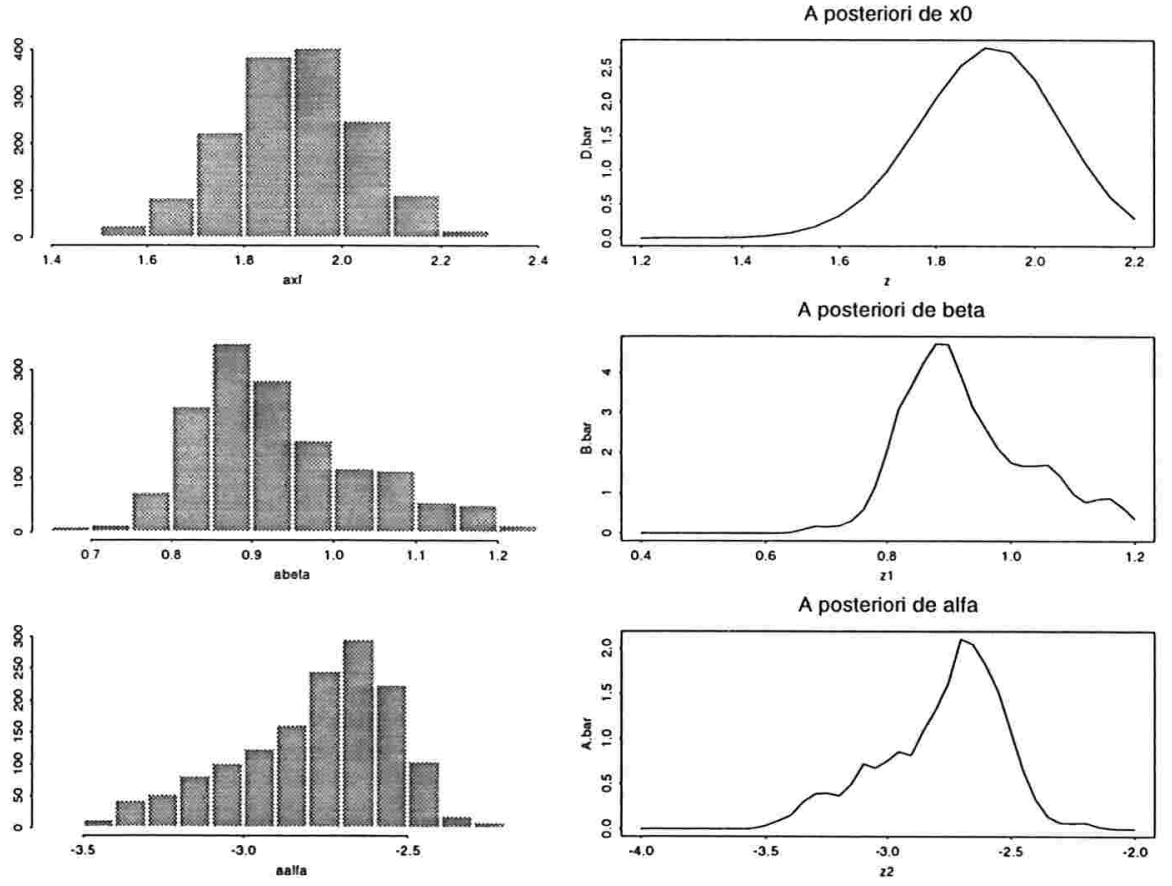

Figura B.3.5: Densidades estimadas para $d_{0}=300$ e $x_{0}=2.48$
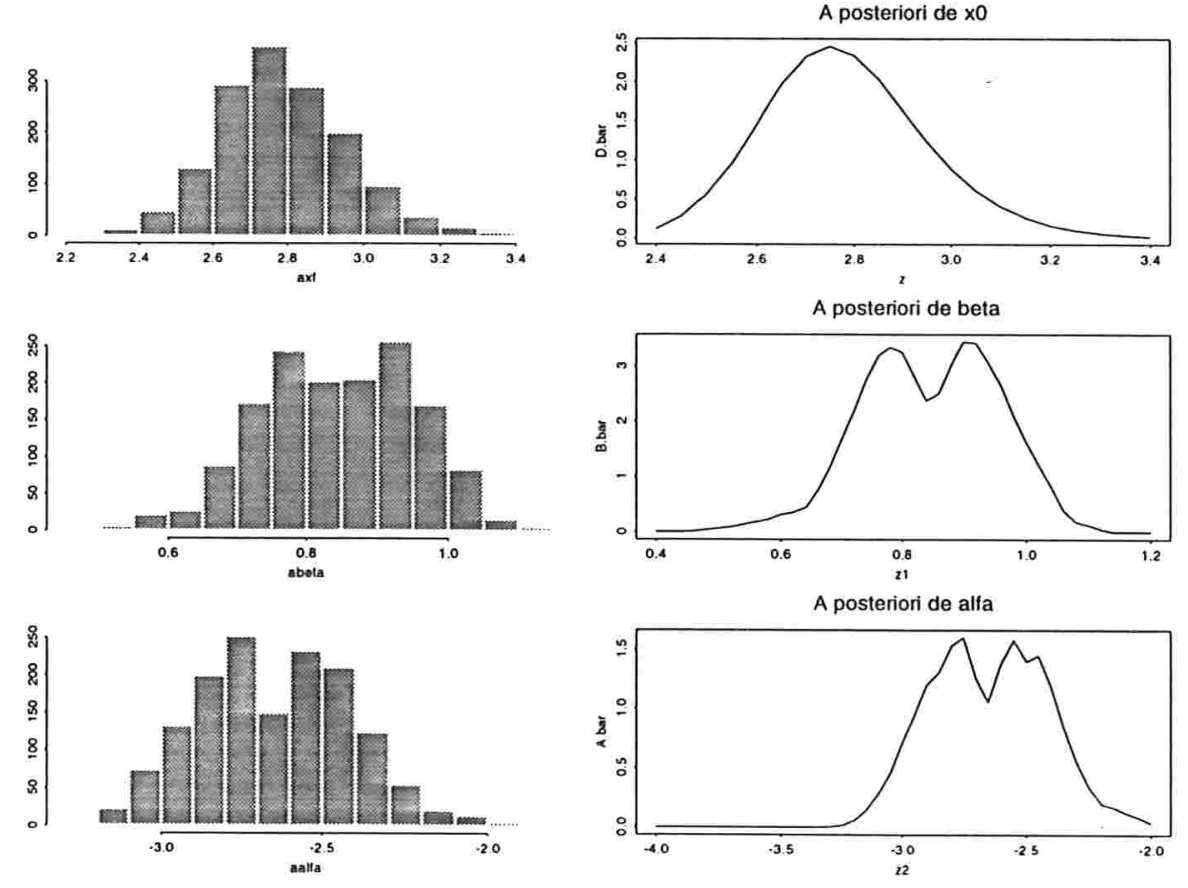
Figura B.3.6: Densidades estimadas para $d_{0}=400$ e $x_{0}=2.60$
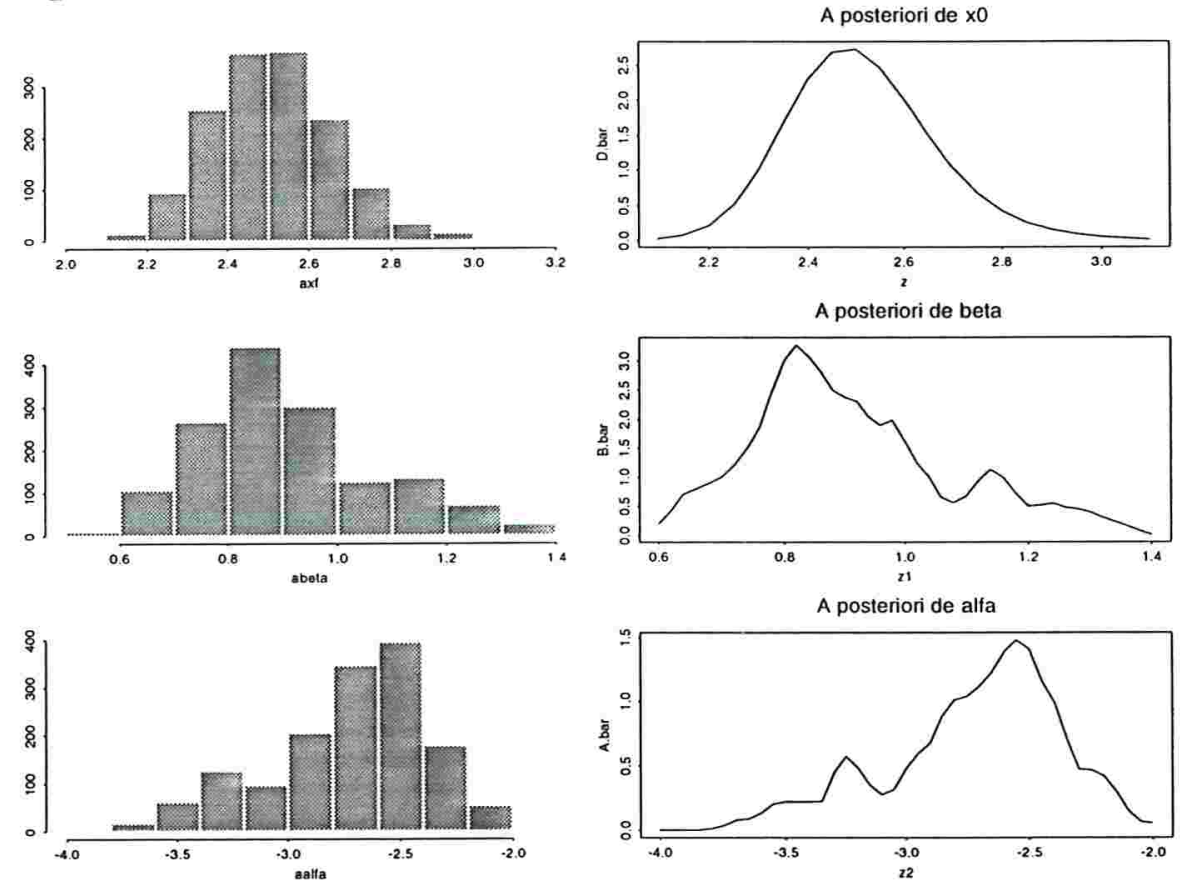


\section{Referências Bibliográficas}

Aitchison, J. and Bennet, J. (1970). Polychotomous quantal response by maximum indicant, Biometrika 57: 253-262.

Aitchison, J. and Silvey, S. (1957). The generalization of probit analysis to the case of multiple responses, Biometrika 44: 131-140.

Albert, J. and Chib, S. (1993). Bayesian analysis of binary and polychotomous response data, JASA 88: 669-679.

Arellano-Valle, R. B. (1994). Distribuições Elipticas: Propriedades, Inferência e Aplicações a Modelos de Regressão, Tese de doutorado - IME-Universidade de São Paulo.

Berkson, J. (1969). Estimation of linear function for a calibration line: consideration of a recent proposal, Technometrics 11: 649-660.

Bernardo, J. (1979). Reference posterior distributions for bayesian inference, JRSS 41: $113-147$.

Bernardo, J. and Smith, A. (1994). Bayesian Theory, John Wiley \& Sons.

Besag, J. and Green, P. (1993). Spatial statistics and bayesian computation, JRSS 55: 2537.

Branco, M., Bofarine, H. and Iglesias, P. (1996). Bayesian calibration under a student-t model, Relatório Técnico, MAE 9623 - IME - USP.

Brown, P. (1982). Multivariate calibration, JRSS 44: 287-321.

Brown, P. (1993). Measurement, Regression and Calibration, Oxford University Press. 
Brown, P. and Sundberg, R. (1987). Confidence and conflict in multivariate calibration, JRSS 49: 46-57.

Chen, C.-F. (1985). On asymptotic normality of limiting density functions with Bayesian implications, JRSS 47: 540-546.

Chow, S. and Shao, J. (1990). On the difference between the classical and inverse methods of calibration, Appl.Statist. 39: 219-228.

Cowles, M. and Carlin, B. (1996). Markov Chain Monte Carlo convergence diagnostics: a comparative review, JASA 91: 883-904.

Dawid, A. (1977). Spherical matrix distributions and multivariate model, JRSS 39: 254261.

Dickey, J. (1967). Multivariate generalizations of the multivariate t-distribution and inverted multivariate t-distribution, Annals of Mathematical Statistics 38: 511-518.

du Plessis, J. and van der Merwe, J. (1994). Inferences in multivariate bayesian calibration, The Statistician 43: 45-60.

Eisenhart, C. (1939). The interpretation of certain regression methods, and their use in biological and industrial research, Annals of Mathematical Statistics 10: 162-186.

Fang, K. and Anderson, T. (1990). Statistical inference in elliptically contoured and related distributions, Allerton Press Inc., New York.

Fang, K. and Zhang, Y. (1990). Generalized Multivariate Analysis, Springer-Verlag.

Fieller, E. (1954). Some problems in interval estimation, JRSS B,16: 175-185.

Finney, D. (1971). Probit Analysis, Cambridge University Press.

Gamerman, D. (1996). Simulaçâo Estocástica via Cadeias de Markov, 12o. SINAPE,ABE.

Gelfand, A., Hills, S., Racine-Poon, A. and Smith, A. (1990). Ilustration of Bayesian inference in normal data models using Gibbs sampling, JASA 85: 972-985.

Gelman, A. (1995). Inference and monitoring convergence, in W.R.Gilks, S.Richardson and D.J.Spiegelharter (eds), Markov Chain Monte Carlo in Practice, New York: Chapman and Hall. 
Gelman, A. and Rubin, D. (1992). Inference from iterative simulation using multiple sequences, Statistical Science 7: 457-511.

Geman, S. and Geman, D. (1984). Stochastic relaxation Gibbs distributions, and the Bayesian restoration of imagens, IEEE PAMI-6: 721-741.

Geweke, J. (1992). Evaluating the accuracy of sampling-based approaches to the calculation of posterior moments, in J. Bernardo (ed.), Bayesian Statistics 4, Oxford:University Press, pp. 625-631.

Geweke, J. (1993). Bayesian treatment of the independent student-t linear model, Journal Appl.Econometrics 8: 519-540.

Goel, P. and DeGroot, M. (1980). Only normal distribution have linear posterior expectations in linear regression, JASA 75: 895-900.

Hasting, W. (1970). Monte Carlo sampling methods using Markov Chains and thei applications, Biometrika 57: 97-109.

Hill, B. M. (1981). Discussion in: A Bayesian analysis of the linear calibration, Technometrics 23: 335-338.

Hoadley, B. (1970). A Bayesian look at inverse linear regression, JASA 65: 356-369.

Hunter, W. and Lamboy, W. (1981). A Bayesian analysis of the linear calibration, Technometrics 23: 323-350.

Kano, Y. (1994). Consistency property of elliptical probability densidade functions, Journal of Multivariate Analysis 51: 139-147.

Krutchkoff, R. (1967). Classical and inverse regression methods of calibration, Technometrics 9: 425-439.

Krutchkoff, R. (1969). Classical and inverse regression methods of calibration in extrapolation, Technometrics 11: 605-608.

Kubokawa, T. and Robert, C. (1994). New perspectives on linear calibration, Journal of Multivariate Analysis 51: 178-200. 
Lange, K., Little, R. and Taylor, J. (1989). Robust statistical modeling using the t distribution, JASA 84: 881-896.

Lieftinck-Koeijers, C. (1987). Multivariate calibration: a generalization of the classical estimator, Journal of Multivariate Analysis 25: 31-44.

Madruga, M., Ochi-Lohmann, T., Okazaki, K. and Pereira, C. (1996). Bayesian dosimetry ii: credibility intervals for radiation dose, Environmetrics 7: 325-331.

Madruga, M. R. (1994). Análise bayesiana de Dados Citogenéticos, Dissertação de mestrado-IME-Universidade de São Paulo.

Mathew, T. and Kasala, S. (1994). An exact confidence region in multivariate calibration, Annals of Statistics 22: 94-105.

McCullang, P. and Nelder, J. (1983). Generalized Linear Models, Chapman and Hall, London.

Metropolis, N., Rosenbluth, A., Rosenbluth, M., Teller, A. and Teller, E. (1953). Equations of state calculations by fast computing machines, Journal of Chemical Physics 21: 1087-1091.

Mood, A., Graybill, F. and Boes, D. (1974). Introduction to the Theory of Statistics, McGraw-Hill.

O'Hagan, A. (1994). Bayesian Inference, London:Edward Arnold.

Oman, S. (1988). Confidence regions in multivariate calibration, Annals of Statistics 16: $174-187$.

Osborne, C. (1991). Statistical calibration: A review, Stat.Review 59: 309-336.

Osiewalski, J. and Steel, M. (1993). Robust Bayesian inference in elliptical regression models, Journal of Econometrics 57: 345-363.

Piegorsch, W. and Casella, G. (1985). The existence of the first negative moment, The American Statistician 39: 60-62.

Press, J. (1984). Applied multivariat analysis, McGraw-Hill. 
Roberts, G. and Smith, A. (1994). Simple conditions for the convergence of the Gibbs sampler and Metropolis-Hastings algorithms, Stoc.Proc.Applic. 49: 207-216.

Smith, A. and Gelfand, A. (1990). Sampling-based aproaches to calculating marginal densities, JASA 85: 398-409.

Smith, A. and Roberts, G. (1993). Bayesian computation via Gibbs sampler and related Markov chain Monte Carlo methods, JRSS 55: 3-23.

Smith, R. and Corbett, M. (1987). Measuring marathon courses:an application of statistical calibration theory, Appl.Stat. 36: 283-295.

Spiegelhalter, D., Thomas, A., Best, N. and Gilks, W. (1994). BUGS: Bayesian Inference Using Gibbs Sampling, version 0.30., Manual.

Tanner, M. (1991). Tools for statistical inference, Lecture Notes in statistics, SpringerVerlag, pp. 1-108.

Tanner, M. and Wong, W. (1987). The calculation of posterior distributions by data augmentation, JASA 82: 528-550.

Tierney, L. (1994). Markov Chains for exploring posterior distributions, Annals of Statistics 22: 1701-1762.

Williams, E. (1969). A note on regression methods in calibration, Technometrics 11: 189192.

Zellner (1976). Bayesian and non-Bayesian analysis of the regression model with multivariate Student-t error term, JASA 71: 400-405. 